\title{
Dynamics of a Passively Mode-Locked Fiber Laser containing a Long-Period Fiber Grating
}

by

\author{
Abdullah S. Karar \\ A Thesis submitted to \\ the Faculty of Graduate Studies and Research \\ in partial fulfilment of \\ the requirements for the degree of \\ Master of Applied Science \\ Ottawa-Carleton Institute for \\ Electrical and Computer Engineering \\ Department of. Electronics \\ Carleton University \\ Ottawa, Ontario, Canada
}

September 2007

Copyright (C)

2007 - Abdullah S. Karar 


$\begin{array}{ll}\begin{array}{l}\text { Library and } \\ \text { Archives Canada }\end{array} & \begin{array}{l}\text { Bibliothèque et } \\ \text { Archives Canada }\end{array} \\ \begin{array}{l}\text { Published Heritage } \\ \text { Branch }\end{array} & \begin{array}{l}\text { Direction du } \\ \text { Patrimoine de l'édition }\end{array} \\ \begin{array}{l}\text { 395 Wellington Street } \\ \text { Ottawa ON K1A ON4 }\end{array} & \begin{array}{l}\text { 395, rue Wellington } \\ \text { Ottawa ON K1A ON4 } \\ \text { Canada }\end{array}\end{array}$

Your file Votre référence

ISBN: 978-0-494-33654-0

Our file Notre référence

ISBN: 978-0-494-33654-0

NOTICE:

The author has granted a nonexclusive license allowing Library and Archives Canada to reproduce, publish, archive, preserve, conserve, communicate to the public by telecommunication or on the Internet, loan, distribute and sell theses worldwide, for commercial or noncommercial purposes, in microform, paper, electronic and/or any other formats.

The author retains copyright ownership and moral rights in this thesis. Neither the thesis nor substantial extracts from it may be printed or otherwise reproduced without the author's permission.
AVIS:

L'auteur a accordé une licence non exclusive permettant à la Bibliothèque et Archives Canada de reproduire, publier, archiver, sauvegarder, conserver, transmettre au public par télécommunication ou par l'Internet, prêter, distribuer et vendre des thèses partout dans le monde, à des fins commerciales ou autres, sur support microforme, papier, électronique et/ou autres formats.

L'auteur conserve la propriété du droit d'auteur et des droits moraux qui protège cette thèse. $\mathrm{Ni}$ la thèse ni des extraits substantiels de celle-ci ne doivent être imprimés ou autrement reproduits sans son autorisation.
In compliance with the Canadian

Privacy Act some supporting forms may have been removed from this thesis.

While these forms may be included in the document page count, their removal does not represent any loss of content from the thesis.
Conformément à la loi canadienne sur la protection de la vie privée, quelques formulaires secondaires ont été enlevés de cette thèse.

Bien que ces formulaires aient inclus dans la pagination, il n'y aura aucun contenu manquant.

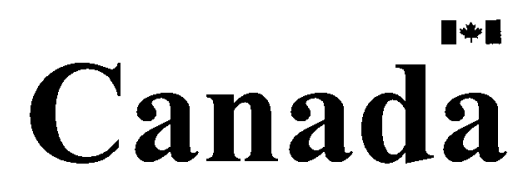




\section{Abstract}

A numerical investigation of the nonlinear dynamics of a passively mode-locked fiber laser containing a long period fiber grating was conducted. The model was based on the complex Ginzburg-Landau equation and the nonlinear coupled mode equations of the grating. The numerical results indicated the existence of passive mode-locking and autosoliton generation in the cavity of the laser. Both single and bound soliton pulse trains were found to exhibit period doubling bifurcations and a route to chaos as the saturated gain was increased. Furthermore, the presence of long period pulsation, soliton sidebands and possible coexisting attractors excited by multisoliton formation and soliton energy quantization was observed. The laser dynamics were presented through plotting the pulse energy against the linearly increasing gain, so obtaining bifurcation diagrams. Higher order nonlinear and dispersive effects, such as Raman self-frequency shift, self-steepening and third order dispersion, were found to change these bifurcation diagrams. 
Dedicated to Dr. Sayed Karar 


\section{Acknowledgments}

I would like to acknowledge the academic and moral support of my supervisor professor Alan Steele and co-supervisor professor Tom Smy. I would like to extend my thanks to Carleton university's department of electronics, the Canadian Foundation for Innovation and Canada's National Science and Engineering Research Council for financial support and availability of high computing facilities. Also, I would like to thank S. Lynch (Manchester Metropolitan University, United Kingdom) and J. Albert (Carleton University, Canada) for their valued input and useful discussions. 


\section{Table of Contents}

Abstract $\quad$ ii

Acknowledgments iv

Table of Contents $\quad$ v

List of Figures viii

1 Introduction 1

1.1 Fiber Lasers . . . . . . . . . . . . . . . . . . . . . 1

1.2 Thesis Motivation . . . . . . . . . . . . . 6

1.3 Contributions .................... 7

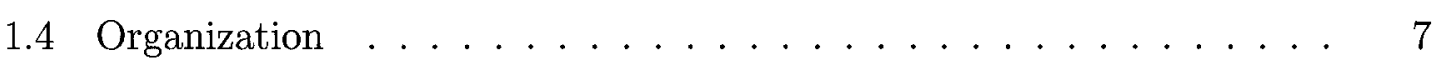

2 Laser Model 9

2.1 Laser System . . . . . . . . . . . . . . . . . . . 9 9

2.2 Fiber Equations . . . . . . . . . . . . . . 11

2.2.1 Maxwell's Equations .................. 11

2.2.2 Nonlinear Pulse Propagation . . . . . . . . . . . . . 13

2.2.3 Erbium-Doped Fiber Amplifier . . . . . . . . . . 18

2.2.4 Fiber Parameters ................. 23

2.3 LPFG Equations . . . . . . . . . . . . . . . 26

$$
\text { V }
$$


2.3.1 Nonlinear Mode Coupling . . . . . . . . . . . . . . 28

$2.3 .2 \quad$ Pulse Shaping . . . . . . . . . . . . . . . . . . . 32

3 Numerical Methods and Implementation 38

3.1 Split-Step Fourier Method . . . . . . . . . . . . . . . . . 39

$3.1 .1 \quad$ Fiber Equation $\ldots \ldots \ldots \ldots \ldots$

$3.1 .2 \quad$ LPFG Equation . . . . . . . . . . . . . . . . . . . 42

3.2 Object Oriented Programming . . . . . . . . . . . . . . 43

4 Laser Dynamics $\quad 48$

4.1 Passive Mode-Locking . . . . . . . . . . . . . . . . . . . 49

4.2 Autosoliton Generation . . . . . . . . . . . . . . . . . . 52

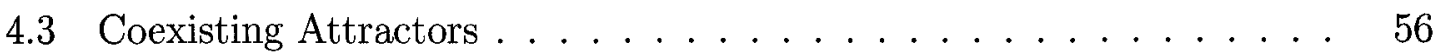

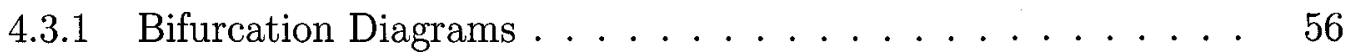

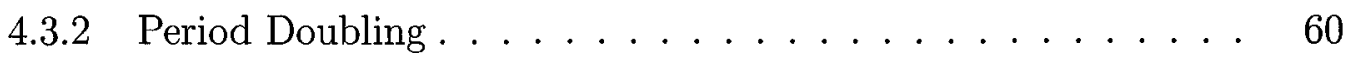

4.3.3 Long Period Pulsation . . . . . . . . . . . . . . . . 61

4.3 .4 Chaos Dynamics . . . . . . . . . . . . . . . . 64

4.4 Dynamics of the LPFG . . . . . . . . . . . . . . . 67

4.5 Soliton Sidebands . . . . . . . . . . . . . . . . . . . 70

5 High Order Effects $\quad 74$

5.1 Higher Order Terms . . . . . . . . . . . . . . . . . 74

5.2 Raman Self-Frequency Shift $\ldots \ldots \ldots \ldots \ldots$

5.3 Self-Steepening $\ldots \ldots \ldots \ldots \ldots \ldots \ldots \ldots \ldots$

5.4 Third Order Dispersion . . . . . . . . . . . . . . . 80

5.5 Ultrashort Pulse Generation . . . . . . . . . . . . . . . 82

6 Conclusion $\quad 84$

vi 
List of References

Appendix A C++ Code 


\section{List of Figures}

1.1 Plot of the number of journal publications on fiber lasers between 19752007. Source: Engineering Village - title search. . . . . . . . . . 2

1.2 Schematic of a Fabry-Perot cavity fiber laser. . . . . . . . . . 4

1.3 Schematic of a unidirectional ring cavity fiber laser. . . . . . . . . 4

1.4 Schematic of a figure of eight ring cavity fiber laser. . . . . . . . . . 4

2.1 Passive mode-locking laser model containing a LPFG. . . . . . . . . 10

2.2 EDFA two energy level model. . . . . . . . . . . . . 19

2.3 Coupling of the core mode into copropagating cladding mode in a 25 mm LPFG written in a single mode fiber (a) schematic. (b) transmission spectrum. . . . . . . . . . . . . . . .

2.4 Transverse refractive index profile for a single mode fiber with core refractive index $n_{1}$ and cladding refractive index $n_{2} . \ldots \ldots$

2.5 Normalized modal profile of the radially symmetric modes $(m=0)$. The fundamental core mode is denoted $\phi_{10}$, while the cladding modes are denoted $\phi_{2-4,0} \ldots \ldots \ldots \ldots \ldots \ldots \ldots$

2.6 LPFG coupling coefficients as a function of the $n^{\text {th }}$ radially symmetric cladding mode for $j=1-20$ (a) linear $d_{1 j}$. (b) nonlinear $c_{1 j}$ and $c_{j j}$. 
2.7 Initial and final pulse profile resulting from propagating a Gaussian pulse in a LPFG. The simulation parameters are $d_{14}=2, c_{11}=c_{44}=$ $0.3, c_{14}=0.5, \Delta=0$ and $\xi_{G}=0.7$ within a computational domain of $T \in[-5,5] \ldots \ldots \ldots \ldots \ldots \ldots$

2.8 Evolution profile of a Gaussian pulse through a LPFG. The simulation parameters are $d_{14}=2, c_{11}=c_{44}=0.3, c_{14}=0.5, \Delta=0$ and $\xi_{G}=0.7$ within a computational domain of $T \in[-5,5]$ (a) core profile. (b)

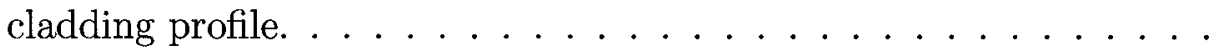

3.1 Illustration of the S-SSFM. The nonlinear operator $\hat{N}$ is evaluated in the midpoint between the linear operators $\hat{D}$ within the segment $\xi$ to

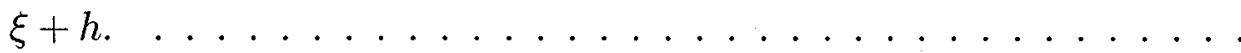

3.2 Simplified UML class diagram for the laser system implementation. . 45

3.3 Program functional blocks with the highest computational time. The Callgrind/KCachegrind output. . . . . . . . . . . . . . . 46

4.1 Stable mode-locked pulse dynamics for a low intensity Gaussian input pulse at $G_{0}=1.2$ (a) intensity of mode-locked pulse after 50 round trips. (b) energy of the mode-locked Gaussian input pulse as a function of cavity round trips.

4.2 Intensity profile of stable mode-locked pulse as a function of cavity round trips for a low intensity Gaussian input pulse at $G_{0}=1.2$ (a) core profile. (b) cladding profile as an output of the LPFG. . . . . . .

4.3 Stable mode-locked pulse dynamics for a low intensity random input pulse at $G_{0}=1.2$ (a) pulse intensity after 200 round trips with an exact soliton solution plotted. (b) energy of the pulse as a function of cavity round trips. . . . . . . . . . . . . . .

4.4 Self-starting autosoliton with a low intensity random light input pulse at $G_{0}=1.2$, with output intensity (a) profile. (b) contour. . . . . . . ix 
4.5 Bifurcation diagram of pulse energy $E$ plotted against the saturated gain for low ASE input (a) $G_{0}=1$ to 4.5 (b) expanded view for $G_{0}=1$ to $3.7 \ldots \ldots \ldots \ldots \ldots$

4.6 Bifurcation diagram of pulse energy $E$ plotted against the saturated gain for high ASE input (a) $G_{0}=1$ to 4.5 (b) expanded view for $G_{0}=$ 1 to $3.5 \ldots \ldots \ldots \ldots \ldots \ldots \ldots$

4.7 Soliton bound states (a) double soliton pulse train at $G_{0}=2.58$ with high ASE input. (b) triple soliton pulse train at $G_{0}=3.58$ with low ASE input. . . . . . . . . . . . . . . . .

4.8 Period doubling of single and bound soliton pulse trains for low ASE input (a) period-2 single soliton pulsation at $G_{0}=2.4$ (b) period-2 double soliton pulsation at $G_{0}=3.4 \ldots \ldots \ldots$

4.9 Long period pulsation at $G_{0}=3.36$ with low ASE input, with output intensity (a) profile. (b) contour. . . . . . . . . . . .

4.10 Chaotic pulsation for a triple soliton pulse train at $G_{0}=4.5$ with high ASE input (a) intensity profile. (b) intensity contour. . . . . . . . . 65

4.11 Pulse energy versus the cavity passes at $G_{0}=4.5$ with high ASE input. 66

4.12 Chaotic secure communication block diagram. . . . . . . . . . . 66

4.13 LPFG Dynamics with cladding light energy $\|V\|^{2}$ and core light energy $\|U\|^{2}$ in the LPFG plotted against laser cavity passes with low ASE input for (a) $G_{0}=1.2$ (b) $G_{0}=3.42 \ldots \ldots 68$

4.14 LPFG Dynamics: (a) cladding light energy $\|V\|^{2}$ and core light energy $\|U\|^{2}$ in the LPFG plotted against laser cavity passes with low ASE input for $G_{0}=4.0$ (b) $\|U\|^{2}$ plotted against $\|V\|^{2}$ for three gain values $G_{0}=1.2,3.42$ and $4.0 \ldots \ldots \ldots \ldots$

4.15 Period doubling temporal soliton pulse at $G_{0}=2.4$ with low ASE input (a) round-1. (b) round-2. 
4.16 Period doubling spectral soliton pulse at $G_{0}=2.4$ with low ASE input (a) round -1 (b) round $-2 \ldots \ldots \ldots \ldots \ldots$

5.1 Laser dynamics with $\tau_{R}=0.1$ and low ASE input (a) bifurcation diagram of pulse energy $E$ plotted against the normalized saturated gain for $G_{0}=1$ to 3 (b) pulse energy $E$ plotted against laser passes at

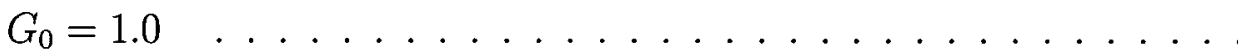

5.2 Laser dynamics with $s=0.03$ and low ASE input (a) bifurcation diagram of pulse energy $E$ plotted against the normalized saturated gain for $G_{0}=1$ to 3 (b) pulse intensity profile plotted against laser passes at $G_{0}=1.0 \ldots \ldots \ldots \ldots$

5.3 Laser dynamics with $\delta=0.03$ and low ASE input (a) bifurcation diagram of pulse energy $E$ plotted against the normalized saturated gain for $G_{0}=1$ to 3 (b) pulse asymmetric spectral intensity at $G_{0}=1.0$

5.4 Bifurcation diagram of pulse energy $E$ plotted against the normalized saturated gain for $G_{0}=1$ to 3 with low ASE input, $\tau_{R}=0.1, s=0.03$

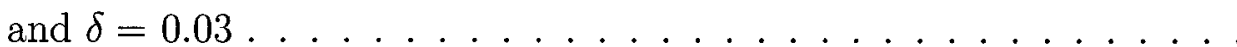




\section{Chapter 1}

\section{Introduction}

The work presented in this thesis investigates the dynamics of a passively mode-locked fiber laser. This chapter provides a brief historical introduction to fiber lasers, with emphasis on passive mode-locking techniques and cavity designs. The motivation and goal of the thesis is also outlined along with all research contributions. Finally, the organization of this document is presented with an overview of each chapter.

\section{$1.1 \quad$ Fiber Lasers}

A laser consists of a gain medium located inside a cavity designed to provide optical feedback. The use of rare-earth ions, such as erbium, neodymium and ytterbium in fiber amplifiers [1] enabled the operation of fiber lasers, where the fiber amplifier is used as the gain medium [2]. Fiber lasers were found to produce higher energy and shorter pulses at low cost in comparison to solid state bulk lasers [3]. The first fiber laser was demonstrated in 1961 [4], with a neodymium-doped fiber segment. As low loss glass fibers became available in 1973, fiber lasers were constructed and pumped using diode lasers [5]. In spite of this early theoretical and experimental research, it was not until the late 1980s that fiber lasers were fully developed after the availability of erbium doped fiber amplifiers (EDFAs) [6]. Alongside this development, the theory 


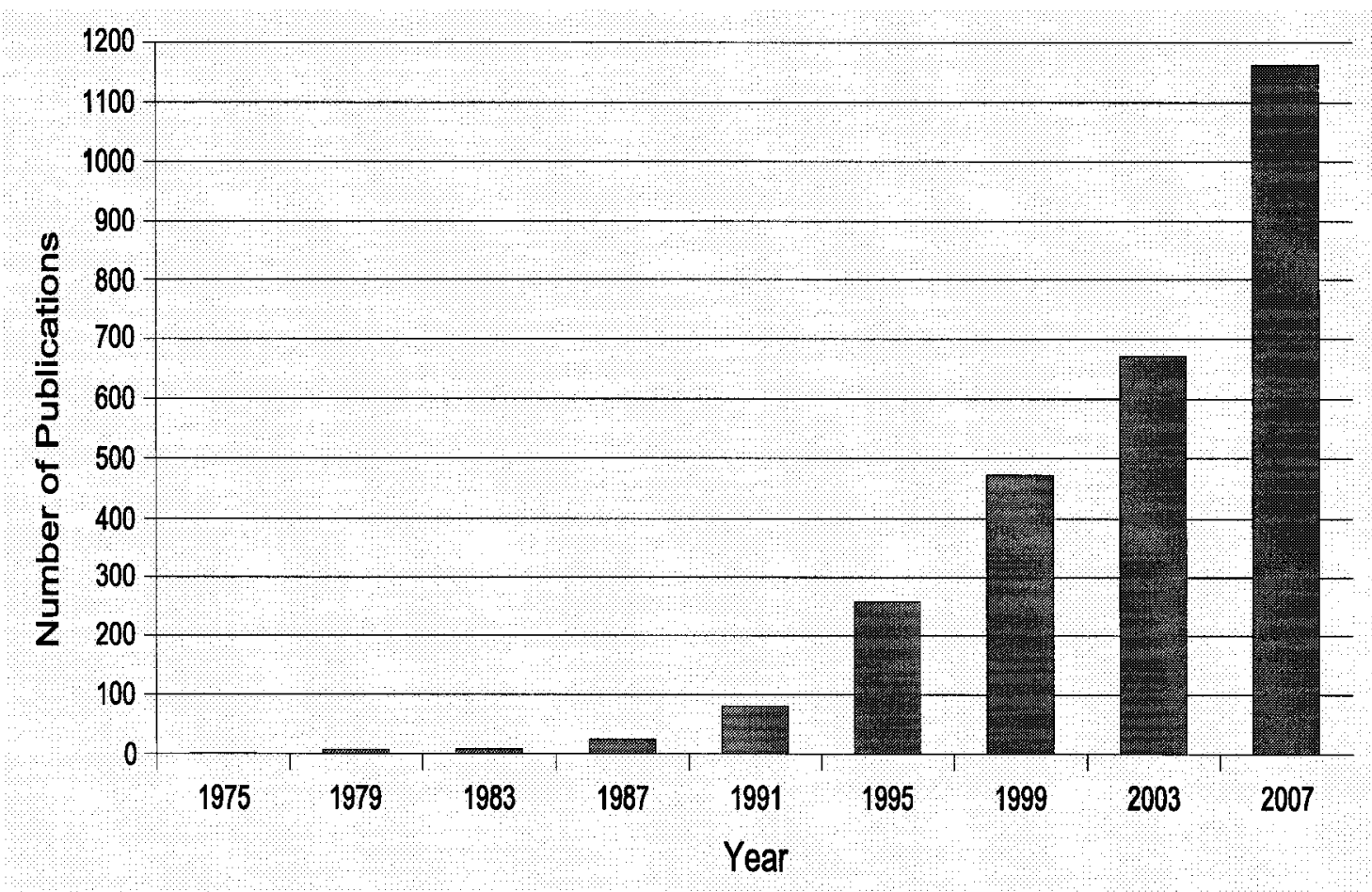

Figure 1.1: Plot of the number of journal publications on fiber lasers between 19752007. Source: Engineering Village - title search.

of mode-locking was established through the work of Lamb as early as 1964 [7]. However, the first mode-locked fiber laser was not demonstrated until 1983 [8]. Fiber lasers can be either actively or passively mode-locked [9]. To explore the progress in the field of fiber lasers, Fig. 1.1 illustrates the number of journal publications on the development and applications of fiber lasers within the past 30 years. A notable increase in research and development can be seen in the early 1990s, as considerable interest has shifted to passively mode-locked erbium-doped fiber lasers, which are capable of producing optical pulses as short as $50 \mathrm{fs}$ at a wavelength of $1550 \mathrm{~nm}$ [2,9]. Passive mode-locking is an all optical technique enabling the laser of producing pulsed light of extremely short duration [3]. There are various methods for passive mode-locking, such as the nonlinear loop mirror method [2], the nonlinear polarization rotation 
(NPR) method [10] and the saturable absorber method $[2,3]$. The simplest technique is the saturable absorber method, which utilizes a passive element adding an intensity discrimination perturbation to the laser cavity [2]. This intensity discrimination process attenuates weaker portions of the individual pulses, after which the gain medium compensates, preserving the total cavity energy. These absorbers are usually liquid organic dyes or doped crystals and semiconductors with intensity dependent transmission properties acting as pulse shaping elements [2,11]. As an example, assuming a simple laser cavity consisting of two high-reflecting plane mirrors facing each other, with the laser gain medium and saturable absorber located between them. An output coupler can be used instead of one of the mirrors. This coupler must be designed such that most of the pump power leaves the port that is part of the laser cavity, such couplers are known as wavelength-division multiplexing (WDM) couplers [12]. This configuration is known as the Fabry-Perot cavity and is shown in Fig. 1.2. As an alternative to this simple configuration, the use of the mirrors and saturable absorber can be eliminated with a unidirectional all-fiber ring cavity, utilizing polarization controllers and an optical isolator as shown in Fig. 1.3. This new configuration allows for two different directions for light to propagate, however, one direction is enforced using the combination of the polarization controllers and the optical isolator. When unidirectional operation is maintained, there is no standing-wave interference pattern and consequently, single-frequency operation is easily achieved [2]. The unidirectional ring fiber laser can be further extended through replacing the gain medium with a nonlinear amplifying loop mirror as shown in Fig. 1.4. The resulting configuration is known as the figure of eight cavity due to its appearance [13]. A full review of more sophisticated cavity designs can be found in [3]. Regardless of cavity design or mode-locking method, fiber lasers have the potential for compact ultrashort pulse operation with low power consumption at a relatively low cost $[2,3]$. 


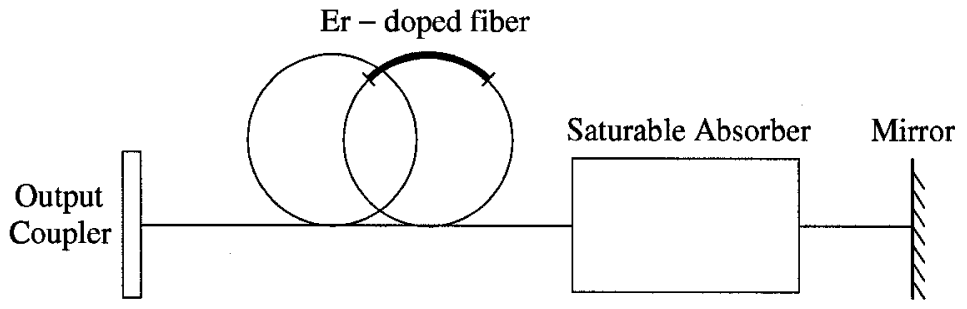

Figure 1.2: Schematic of a Fabry-Perot cavity fiber laser.

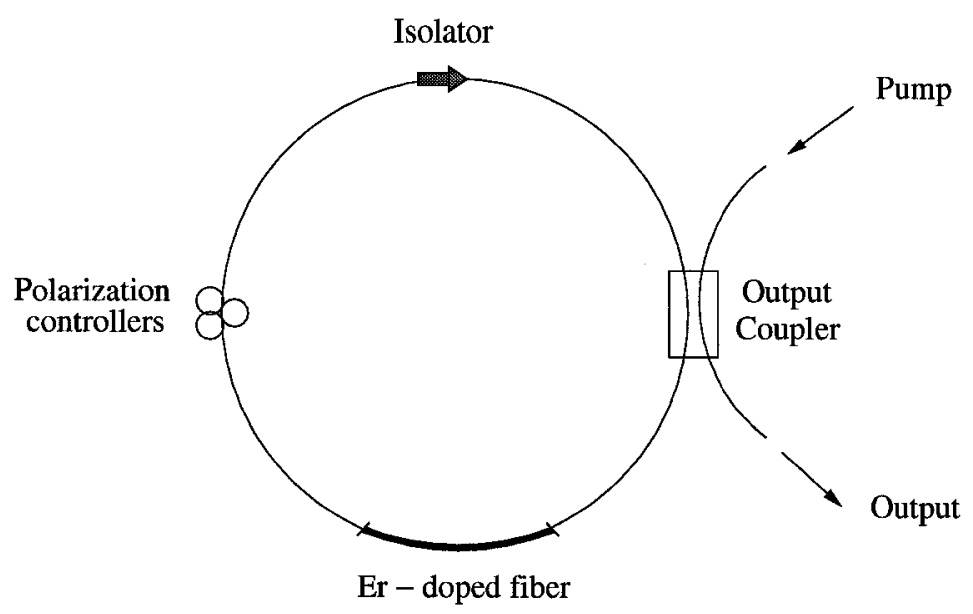

Figure 1.3: Schematic of a unidirectional ring cavity fiber laser.

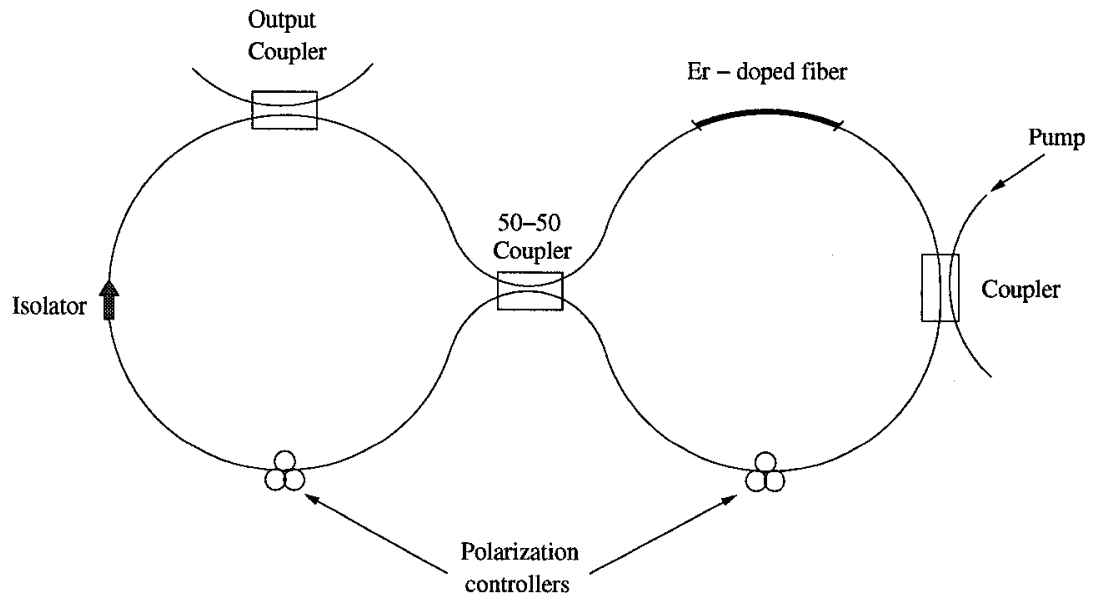

Figure 1.4: Schematic of a figure of eight ring cavity fiber laser. 
Independent of the mode-locking technique, fiber lasers were found to generate soliton-like pulses $[2,9,14]$. The word soliton refers to waves, that have particle like properties, propagating in a nonlinear medium [15]. Soliton waves have a shape preserving property and can undergo elastic collisions [16]. The presence of solitons in optical fiber and their use in optical communication were reported in 1973 [17], and observed experimentally by 1980 [18]. Light propagating in a nonlinear medium with dispersion has been conventionally modeled using the nonlinear Schrödinger equation (NLSE), where soliton solutions arise from the balance between group velocity dispersion and self-phase modulation [16]. However, as lasers incorporate a gain medium the soliton operation of passively mode-locked lasers has been conventionally modeled using the modified NLSE, also known as the complex Ginzburg Landau equation (CGLE), which incorporates fiber nonlinearity, dispersion, loss and amplifier gain $[2,9]$. The solutions of the CGLE are known as solitary-waves or autosolitons [19], due to their self-starting property [20]. Although these soliton-like pulses are inherently stable, laser models based in the CGLE were found to exhibit a range of nonlinear phenomena including period doubling bifurcation route to chaos, multisoliton formation and energy quantization [21-23]. These dynamics were reported experimentally and numerically in fiber lasers, such as the figure of eight laser [24], and the NPR laser [25]. In addition, the coexistence of multiple attractors, a general trend in dissipative driven nonlinear systems [26], has also been observed in mode-locked lasers. For example, a $\mathrm{CO}_{2}$ laser with modulated losses has shown to exhibit multiple attractors for the same set of system parameters [27]. A generalized multistability analysis of erbium-doped fiber lasers with modulated losses based on rate equations also show coexisting attractors for photon density [28]. Experimental work on erbium-doped fiber laser with a sinusoidal pump modulation also shows the presence of coexisting attractors [29,30]. Further, the existence of long-period pulsation [21] and soliton sidebands [2,22] have also been reported in fiber lasers. 


\subsection{Thesis Motivation}

Ultrashort pulse laser systems have been used in a variety of applications ranging from elemental mass spectrometry to telecommunications $[11,31]$. Passive mode-locked fiber lasers incorporating erbium-doped fibers have emerged as low cost ultrashort pulse sources $[2,9]$. Instabilities in mode-locked laser pulse generation may hinder its functionality, especially for critical applications where accuracy is vital. Hence, stability analysis plays an increasingly important role in laser design and modeling.

In this research project, a numerical investigation of the nonlinear dynamics and instabilities of a passively mode-locked fiber laser containing a long period fiber grating (LPFG) is conducted. The theoretical use of the LPFG as a pulse shaping mechanism, instead of the saturable absorber in Fig. 1.2, was first proposed by K. Intrachat and J. N. Kutz [32]. The laser model in [32], was based on the CGLE and the nonlinear coupled mode equations of the LPFG. However, their work did not investigate the range of nonlinear dynamics exhibited in this type of laser. This work reports on the existence of self-starting, autosolitons in the cavity of the laser, with both single and bound soliton pulse trains exhibiting period doubling bifurcations and an eventual route to chaos as the normalized saturated gain is increased. Furthermore, the presence of long period pulsation, soliton sidebands and coexisting attractors excited by multisoliton formation and soliton energy quantization is evident. The original Kutz model was further extended, to include higher order nonlinear and dispersive and effects, such as Raman self-frequency shift, self-steepening and third order dispersion. In this work, the CGLE as it stands alone, has no mode-locking term and hence does not enable mode-locking. It is the combination of the CGLE and the nonlinear mode coupling equations of the LPFG that allow the laser model to mode-lock. Thus, the wide range of dynamics observed is an intrinsic property of the soliton laser system regardless of the cavity model used. 


\subsection{Contributions}

- A. S. Karar, T. Smy and A. L. Steele, "Nonlinear dynamics of a passively modelocked fiber laser containing a long-period fiber grating," submitted to IEEE J. Quantum Electron., JQE-131029, 2007

- A. S. Karar, T. Smy and A. L. Steele, "Raman self-frequency shift in a passively mode-locked fiber laser containing a long period fibre grating," poster session to be presented at the Bragg Gratings, Photosensitivity and Poling in Glass Waveguides and Nonlinear Photonics meeting to be held at Quebec City, Quebec, Canada, Sept. 2007

- A. S. Karar, A. L. Steele and T. Smy, "Object-oriented numerical simulation of a passively modelocked fiber laser," paper presented at the Photonics North meeting held in Ottawa, Ontario, Canada, June 2007

- A. S. Karar, A. L. Steele. A set of software functions developed to model the laser in both MATLAB [33] and C++ [34] (available upon request).

\subsection{Organization}

This thesis is organized in the following manner. Chapter 2 presents the laser model with an appropriate introduction to nonlinear pulse propagation in optical fiber. The NLSE is derived from Maxwell's equations and extended to the CGLE for the purpose of modeling EDFAs. Furthermore, the nonlinear coupled mode equations of the LPFG are presented and simulated to illustrate pulse shaping.

Chapter 3 gives a brief description of the numerical methods employed in solving the CGLE and the nonlinear coupled mode equations of the LPFG. The basic, symmetric and predictor-corrector split step Fourier methods are introduced. Further, an 
object-oriented approach is adapted in coding the numerical algorithms. The merits and demerits of using object orientation are also discussed.

Chapter 4 reports on the dynamics of the laser. Passive mode-locking is demonstrated with a seeded Gaussian light input. Autosoliton generation is demonstrated with a random light input. The coexistence of multiple attractors excited by multisoliton formation is shown through plotting the pulse energy against the linearly increasing gain, so obtaining bifurcation diagrams. The nonlinear dynamics observed on each attractor are examined, such as period doubling, long period pulsation, chaos and soliton sidebands. The dynamics within the LPFG are also discussed.

Chapter 5 extends the original Kutz model to include higher order nonlinear and dispersive effects, such as Raman self-frequency shift, self-steepening and third order dispersion.

Chapter 6 summarizes the findings obtained in this thesis and predicts a possible direction this investigation may move on to in the future.

Appendix A contains synopsis from the $\mathrm{C}++$ code. 


\section{Chapter 2}

\section{Laser Model}

The fiber laser investigated in this thesis follows the configuration proposed by $\mathrm{K}$. Intrachat and J. N. Kutz [32]. The laser has a Fabry-Perot cavity, with a nonlinear gain saturable EDFA as the gain medium and a LPFG as the pulse shaping element. In this chapter, the propagation equations of light in the EDFA is derived from Maxwell's equations. Further, the nonlinear coupled mode equations of the LPFG are introduced and the pulse shaping mechanism is demonstrated.

\subsection{Laser System}

The cavity of the modeled laser is composed of an ideal reflective mirror, a short section of LPFG, a nonlinear gain saturable fiber and an output coupler as shown in Fig. 2.1. The fiber, in practice, consists of a section of single-mode fiber with a spliced section of erbium-doped fiber for cavity gain amplification [32]. From a physical stand point, mode-locking of lasers requires the combination of two effects. Specifically, an intensity discrimination within the laser cavity in conjunction with a bandwidth limited gain [9]. As a consequence of the intensity discrimination, weaker portions of the pulse wings are attenuated, while the pulse peak is transmitted efficiently. The gain medium compensates this attenuation preserving the total cavity 


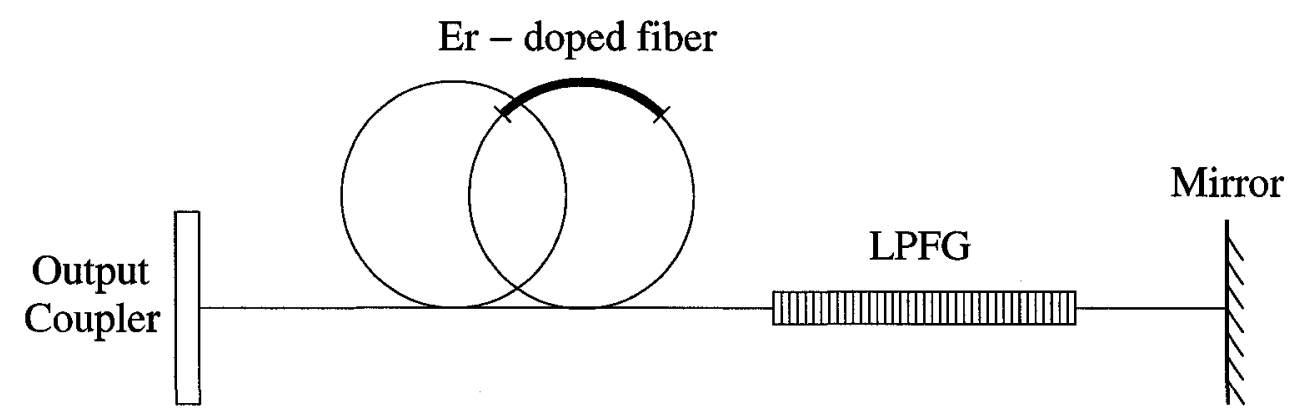

Figure 2.1: Passive mode-locking laser model containing a LPFG.

energy. However, the net gain per round trip for the pulse peak is higher than that for the pulse wings resulting in a pulse shaping effect [2]. Conventionally, mode-locked fiber lasers have been modeled using perturbations of the NLSE with the intention of capturing two key effects [16]. First, the balance between gain and loss resulting from the presence of the gain medium and the intensity discrimination. Second, the balance between nonlinearity and dispersion forming stable pulses [32]. The resulting equation is known as the CGLE, which is a non-Hamiltonian generalization of the NLSE [21]. In most studies of mode-locked lasers, the CGLE contains a phenomenological intensity discrimination term which leads to the stable development of mode-locked pulse trains. The resulting model is known as the master mode-locking equation $[9,23]$. However, in this study the CGLE is adapted for modeling the EDFA without any intensity discrimination term, as the intensity dependent loss is achieved through the nonlinear mode coupling equations of the LPFG [35].

Mathematically, the laser of Fig. 2.1, has two main set of equations. The first is the CGLE and the other is the nonlinear mode coupling equations of the LPFG. As the soliton-like dynamics arise from light circulating the cavity in a round trip fashion, the solution of one set of equations is used as the initial input to the other. For this to be possible, both set of equations must have the same normalization. The following sections are dedicated to introducing and normalizing these equations. 


\subsection{Fiber Equations}

In this section, a full derivation of the appropriate evolution equations for optical pulses in the fiber amplifier is presented. Understanding the nonlinear pulse propagation in the EDFA segment involves two steps. First, the NLSE is derived from Maxwell's equations. Then, the propagation behavior in the presence of a gain medium is presented through extending the NLSE to a normalized CGLE.

\subsubsection{Maxwell's Equations}

Maxwell's equations for electromagnetic radiation are the most accurate model, in classical physics, describing light. Using these equations optical systems can, in theory, be modeled exactly. However, as the complexity of these systems increase, e.g. mode-locked lasers, approximations are necessary, and analytical or numerical solutions are sought. For example, to describe nonlinear propagation in optical fibers, one or a set of coupled partial differential equations based on Maxwell's equations can be used, also known as NLSE. Maxwell's equations in SI units are given as [36]:

$$
\begin{aligned}
\nabla \times \mathbf{E} & =-\frac{\partial \mathbf{B}}{\partial t} \\
\nabla \times \mathbf{H} & =\mathbf{J}_{f}+\frac{\partial \mathbf{D}}{\partial t} \\
\nabla \cdot \mathbf{D} & =\rho_{f} \\
\nabla \cdot \mathbf{B} & =0
\end{aligned}
$$

where $\mathbf{E}$ and $\mathbf{H}$ denote the electric and magnetic field vectors and $\mathbf{D}$ and $\mathbf{B}$ denote the corresponding electric and magnetic flux densities. The current density vector $\mathbf{J}_{f}$ and the charge density $\rho_{f}$ are both set to zero due to the absence of free charge in glass fibers [37]. The electric and magnetic flux densities are related to their corresponding 
field vectors through the following relationships [16]:

$$
\begin{aligned}
& \mathbf{D}=\varepsilon_{0} \mathbf{E}+\mathbf{P} \\
& \mathbf{B}=\mu_{0} \mathbf{H}+\mathbf{M}
\end{aligned}
$$

where $\varepsilon_{0}$ and $\mu_{0}$ denote vacuum permittivity and permeability, respectively and $\mathbf{P}$ and $\mathbf{M}$ are the induced electric and magnetic polarizations. In optical fiber $\mathbf{M}=0$ as glass silica is a nonmagnetic medium $[16,37]$.

Light propagation in optical fiber can be described through the wave equation, which is obtained through taking the curl of Eq.(2.1), while substituting B with Eq.(2.6) and using Eqs.(2.2) and (2.5) [16]. This will result in a wave equation in terms of only the electric field and polarization given by:

$$
\nabla \times \nabla \times \mathbf{E}=-\frac{1}{c^{2}} \frac{\partial^{2} \mathbf{E}}{\partial t^{2}}-\mu_{0} \frac{\partial^{2} \mathbf{P}}{\partial t^{2}}
$$

where $c$ is the velocity of light in the vacuum. In linear media the induced polarization is proportional to the electric field. However, in optical fibers, the light intensity can be high, especially for ultrashort pulses, and higher order polarization terms have to be included [16]. Usually, quantum mechanical perturbation theory of the atomic wave function is used in describing the evolution of resonant induced polarization [38]. However, when the optical frequency is far from the medium resonance, the induced polarization can be written as a sum of linear and nonlinear contributions $[16,38]$ :

$$
\mathbf{P}=\mathbf{P}_{L}+\mathbf{P}_{N L}=\varepsilon_{0}\left(\chi^{(1)} \cdot \mathbf{E}+\chi^{(2)}: \mathbf{E E}+\chi^{(3)}: \mathbf{E E E}+\ldots\right)
$$

where $\chi^{(1)}$ is the linear susceptibility and $\chi^{(n)}$ is the nonlinear susceptibility, given that $n>1$ and $\chi^{(n)}: \mathbf{E E} \ldots \mathbf{E}_{n}$ is a tensor product. The real part of $\chi^{(1)}$ is related to the real part of the refractive index of the medium, while the imaginary part relates to 
the medium loss and gain. The real part of $\chi^{(2)}$ relates to second harmonic generation which is set to zero is glass fibers, due to inversion symmetry in silica [38]. Thus, all even terms in Eq.(2.8) are eliminated leaving $\chi^{(3)}$ as the only significant nonlinear term. Hence, the nonlinear induced polarization can be expressed as:

$$
\mathbf{P}_{N L}=\varepsilon_{0} \chi^{(3)}: \mathbf{E E E}
$$

where $\chi^{(3)}$ governs third order nonlinear effects, such as Kerr nonlinearity, Raman scattering, third harmonic generation and four-wave mixing [38].

\subsubsection{Nonlinear Pulse Propagation}

In this section, following the work shown in [16], a derivation of the basic equation governing optical pulse propagation in nonlinear dispersive fibers is presented. First, we start with Eq.(2.7), and using the following identity:

$$
\nabla \times \nabla \times \mathbf{E}=\nabla(\nabla \cdot \mathbf{E})-\nabla^{2} \mathbf{E}=-\nabla^{2} \mathbf{E}
$$

under the assumption that the refractive index is independent of the spatial coordinates within a local region [16], the wave equation can be expressed as:

$$
\nabla^{2} \mathbf{E}-\frac{1}{c^{2}} \frac{\partial^{2} \mathbf{E}}{\partial t^{2}}=\mu_{0} \frac{\partial^{2} \mathbf{P}_{L}}{\partial t^{2}}+\mu_{0} \frac{\partial^{2} \mathbf{P}_{N L}}{\partial t^{2}}
$$

Here several assumptions are made to simplify solving Eq.(2.11). First, it is reasonable to assume that the nonlinear induced polarization $\mathbf{P}_{N L}$ is a perturbation to $\mathbf{P}_{L}$, as the nonlinear changes in refractive index are less than $10^{-6}$ for silica. Second, in order for a scalar approach to be valid, the optical field is assumed to maintain its polarization as it propagates along the fiber length. Finally, a quasi-monochromatic field is assumed with a pulse spectrum centred at $\omega_{0}$, with spectral width $\Delta \omega$, such that $\Delta \omega / \omega_{0}<<1$. 
As a consequence, a slowly varying envelope approximation (SVEA) is used, and is valid over the optical telecommunication wavelength for pulses as short as 0.1 ps. This SVEA is applied to both electric field and induced polarizations as follows [16]:

$$
\begin{aligned}
\mathbf{E}(\mathbf{r}, t) & =\frac{1}{2} \hat{x}\left[E(\mathbf{r}, t) \exp \left(-i \omega_{0} t\right)+c . c .\right] \\
\mathbf{P}_{L}(\mathbf{r}, t) & =\frac{1}{2} \hat{x}\left[P_{L}(\mathbf{r}, t) \exp \left(-i \omega_{0} t\right)+c . c .\right] \\
\mathbf{P}_{N L}(\mathbf{r}, t) & =\frac{1}{2} \hat{x}\left[P_{N L}(\mathbf{r}, t) \exp \left(-i \omega_{0} t\right)+c . c .\right]
\end{aligned}
$$

where $E(\mathbf{r}, t)$ changes slowly with time, $\hat{x}$ is the polarization unit vector and c.c. denotes complex conjugate. Using this SVEA, the nonlinear response of the medium has to be instantaneous for Eq.(2.9) to hold. Consequently, light's interaction with optical phonons (Raman scattering) contribution to $\chi^{(3)}$ is neglected. Raman scattering will be incorporated later. Now, it is possible to express $\mathbf{P}_{N L}$ in terms of $E(\mathbf{r}, t)$. This is done through substituting Eq.(2.12) in Eq.(2.9), which is rewritten as:

$$
\mathbf{P}_{N L}=\varepsilon_{0} \chi^{(3)}: \frac{\hat{x}}{8}\left[E E E \exp \left(-i 3 \omega_{0} t\right)+3 E E \bar{E} \exp \left(-i \omega_{0} t\right)+c . c .\right]
$$

It is evident from Eq.(2.15) that $\mathbf{P}_{N L}$ has two terms oscillating at $\omega_{0}$ and $3 \omega_{0}$. The third harmonic requires phase matching and is usually neglected in optical fibers [16]. Using Eq.(2.14), $P_{N L}$ can be defined in terms of dielectric constants as follows:

$$
P_{N L} \approx \varepsilon_{0} \varepsilon_{N L} E(\mathbf{r}, t)
$$

with the nonlinear dielectric constant $\varepsilon_{N L}$ defined as $[16,38]$ :

$$
\varepsilon_{N L}=\frac{3}{4} \chi_{x x x x}^{(3)}|E(\mathbf{r}, t)|^{2}
$$

It is clear that $\varepsilon_{N L}$ is intensity dependent, which is a result of Kerr nonlinearity [38]. 
Following [16], the derivation of the wave equation for the SVEA of $E(\mathbf{r}, t)$ is more convenient in the Fourier domain. However, the intensity dependence of $\varepsilon_{N L}$ will make this impossible. This can be overcome through assuming a constant $\varepsilon_{N L}$ during propagation $[12,38]$. This is a reasonable approximation, given the fact that $P_{N L}<<P_{L}$. Now substituting Eqs.(2.12)-(2.14) in the original wave equation given in Eq.(2.11), results in the Fourier transfrom of $E(\mathbf{r}, t)$ which is defined as:

$$
\widetilde{E}\left(\mathbf{r}, \omega-\omega_{0}\right)=\int_{-\infty}^{\infty} E(\mathbf{r}, t) \exp \left[i\left(\omega-\omega_{0}\right) t\right] d t
$$

being a solution to the Helmholtz equation of the form:

$$
\nabla^{2} \widetilde{E}+\varepsilon(\omega) k_{0}^{2} \widetilde{E}=0
$$

where $k_{0}=\omega_{0} / c$ is the wavenumber and the dielectric constant $\varepsilon(\omega)$ is given by [16]:

$$
\varepsilon(\omega)=1+\widetilde{\chi}_{x x}^{(1)}(\omega)+\varepsilon_{N L}
$$

which also can be written in terms of refractive index $\widetilde{n}$ and absorption coefficient $\widetilde{\alpha}$ :

$$
\varepsilon(\omega)=\left(\widetilde{n}+\frac{i \widetilde{\alpha} c}{2 \omega}\right)^{2}
$$

It is clear that both $\widetilde{n}$ and $\widetilde{\alpha}$ are intensity dependent due to the intensity dependence (Kerr nonlinearity) of $\varepsilon_{N L}$ and thus can be separated into two parts as [16]:

$$
\begin{aligned}
& \widetilde{n}=n+n_{2}|E|^{2} \\
& \widetilde{\alpha}=\alpha+\alpha_{2}|E|^{2}
\end{aligned}
$$

where $n_{2}$ denotes the nonlinear refractive index and is used as measure of nonlinearity. 
Using Eq.(2.17) and Eqs.(2.22) and (2.23), $n_{2}$ and $\alpha_{2}$ can be written as:

$$
\begin{aligned}
n_{2} & =\frac{3}{8 n} \operatorname{Re}\left(\chi_{x x x x}^{(3)}\right) \\
\alpha_{2} & =\frac{3 \omega_{0}}{4 n c} \operatorname{Im}\left(\chi_{x x x x}^{(3)}\right)
\end{aligned}
$$

the effect of $\alpha_{2}$ (two-photon absorption coefficient) in silica fibers is small and thus can be neglected [38]. For silica fibers, the nonlinear refractive index, $n_{2}=2.6 \times$ $10^{-20} \mathrm{~m}^{2} / \mathrm{W}$ at $1.55 \mu \mathrm{m}$ [39]. In order to solve Eq.(2.19), the slowly varying part of the electric field $\widetilde{E}\left(\mathbf{r}, \omega-\omega_{0}\right)$ is separated into a product of a function with transversal dependence $F(x, y)$ and a function with longitudinal dependence $A(z)$ as shown [16]:

$$
\widetilde{E}\left(\mathbf{r}, \omega-\omega_{0}\right)=F(x, y) \widetilde{A}\left(z, \omega-\omega_{0}\right) \exp \left(i \beta_{0} z\right)
$$

where $\beta_{0}$ is the wavenumber. The Helmholtz equation is now transformed to two separate differential equations, one in the transversal dependent function and the other in the longitudinal dependent function:

$$
\begin{aligned}
\frac{\partial^{2} F}{\partial x^{2}}+\frac{\partial^{2} F}{\partial y^{2}}+\left[\varepsilon(\omega) k_{0}^{2}-\widetilde{\beta}^{2} F\right] & =0 \\
2 i \beta_{0} \frac{\partial \widetilde{A}}{\partial z}+\left(\widetilde{\beta}^{2}-\beta_{0}^{2}\right) \widetilde{A} & =0
\end{aligned}
$$

the modal distribution $F(x, y)$ will be required in calculating the LPFG coupling coefficients in the next section. Equation (2.27) can be solved using first-order perturbation theory [16], with the eigenvalue $\widetilde{\beta}$ becoming:

$$
\begin{gathered}
\widetilde{\beta}(\omega)=\beta(\omega)+\Delta \beta \\
\Delta \beta=k_{0} \frac{\iint_{-\infty}^{\infty} \Delta n|F(x, y)|^{2} d x d y}{\iint_{-\infty}^{\infty}|F(x, y)|^{2} d x d y}
\end{gathered}
$$


where $\Delta n$ is a small perturbation given below, assuming a constant $n_{2}$ in both core and cladding [16]:

$$
\Delta n=n_{2}|E|^{2}+\frac{i \widetilde{\alpha}}{2 k_{0}}
$$

Using Eq.(2.29) and substituting $\widetilde{\beta}^{2}-\beta_{0}^{2}$ by $2 \beta_{0}\left(\widetilde{\beta}-\beta_{0}\right)$, Eq. $(2.28)$ is rewritten as [16]:

$$
\frac{\partial \widetilde{A}}{\partial z}=i\left(\beta(\omega)+\Delta \beta-\beta_{0}\right) \widetilde{A}
$$

where no exact solution for $\beta(\omega)$ exists and a Taylor expansion around $\omega_{0}$ is necessary:

$$
\begin{gathered}
\beta(\omega)=\beta_{0}+\left(\omega-\omega_{0}\right) \beta_{1}+\frac{1}{2}\left(\omega-\omega_{0}\right)^{2} \beta_{2}+\frac{1}{6}\left(\omega-\omega_{0}\right)^{3} \beta_{3}+\ldots \\
\beta_{m}=\left(\frac{d^{m} \beta}{d \omega^{m}}\right)_{\omega=\omega_{0}}(m=1,2, \ldots)
\end{gathered}
$$

Here, terms with $m \geq 3$ can be neglected for SVEA, since $\Delta \omega / \omega_{0} \ll 1$. However, third order dispersion $(m=3)$ will be incorporated later on in the investigation. Substituting Eq.(2.33) with $m=1,2$ in Eq.(2.32) we obtain:

$$
\frac{\partial \widetilde{A}}{\partial z}=i\left(\left(\omega-\omega_{0}\right) \beta_{1}+\frac{1}{2}\left(\omega-\omega_{0}\right)^{2} \beta_{2}+\Delta \beta\right) \widetilde{A}
$$

at this stage it is possible to use an inverse Fourier transform on Eq.(2.34), while replacing $\left(\omega-\omega_{0}\right)$ with $i(\partial / \partial t)$ and $\left(\omega-\omega_{0}\right)^{2}$ with $-\left(\partial^{2} / \partial t^{2}\right)$. This results in:

$$
\frac{\partial A}{\partial z}=-\beta_{1} \frac{\partial A}{\partial t}-\frac{i \beta_{2}}{2} \frac{\partial^{2} A}{\partial t^{2}}+i \Delta \beta A
$$

where the term $\Delta \beta$ governs the effect of fiber loss and nonlinearity. It can be evaluated through substituting Eq.(2.31) in Eq.(2.30), with $\alpha_{2}=0$, resulting in $\Delta \beta$ being:

$$
\Delta \beta=\frac{i \alpha}{2}+\gamma|A|^{2}
$$


where the fiber nonlinear parameter $\gamma$ is defined as:

$$
\gamma=\frac{n_{2} \omega_{0}}{c A_{e f f}}
$$

where $A_{\text {eff }}$ is the effective core area and is defined by the overlap integral [39]:

$$
A_{e f f}=\frac{\left(\iint_{-\infty}^{\infty}|F(x, y)|^{2} d x d y\right)^{2}}{\iint_{-\infty}^{\infty}|F(x, y)|^{4} d x d y}
$$

Finally, substituting Eq.(2.36) in Eq.(2.35) results in the following nonlinear propagation equation also known as the NLSE:

$$
\frac{\partial A}{\partial z}+\beta_{1} \frac{\partial A}{\partial t}+\frac{i \beta_{2}}{2} \frac{\partial^{2} A}{\partial t^{2}}+\frac{\alpha}{2} A=i \gamma|A|^{2} A
$$

However, the laser model presented in Fig. 2.1 utilizes an erbium-doped fiber section and the NLSE does not include gain, perventing it from modeling propagation in EDFAs. The following section extends the NLSE to be able to handle amplifier gain.

\subsubsection{Erbium-Doped Fiber Amplifier}

Passive mode-locked fiber lasers incorporating erbium-doped fibers have emerged as simple ultrashort pulse sources [2,9]. As a result of minimal optical loss at $1550 \mathrm{~nm}$, the use of EDFAs in lightwave systems has gained momentum. Following the work done in [40], the NLSE, which is useful for describing nonlinear pulse propagation in undoped fiber, will be extended here to include gain resulting from dopants.

Stimulated emission is the main light amplification mechanism in EDFAs. In general, an optical pump excites dopant electrons to higher energy levels creating a population inversion. This work only considers the two energy levels participating in light-induced transitions as shown in Fig. 2.2. The gain $g$ would be calculated as 


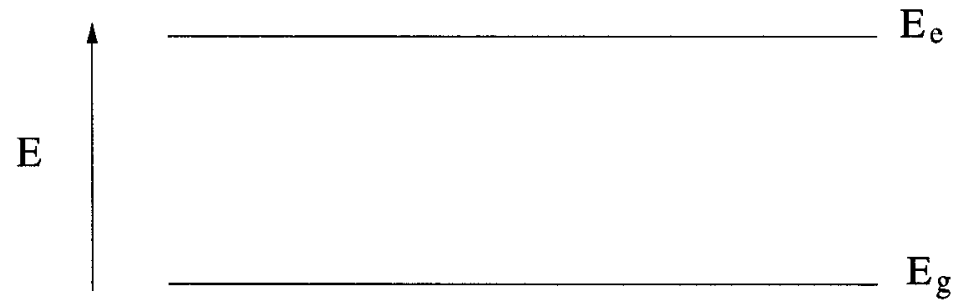

Figure 2.2: EDFA two energy level model.

$\sigma\left(N_{1}-N_{2}\right)$, where $\sigma$ is the transition cross section and $N_{1}$ and $N_{2}$ are the atomic densities in the ground energy state $E_{g}$ and the excited energy state $E_{e}$ respectively. The system in Fig. 2.2 is governed by Maxwell-Bloch equations [1,41]. The induced polarization of Eq.(2.8) has to accommodate a third term $\mathbf{P}_{d}(\mathbf{r}, t)$ as:

$$
\mathbf{P}=\mathbf{P}_{L}+\mathbf{P}_{N L}+\mathbf{P}_{d}
$$

where $\mathbf{P}_{d}$ relates to the dopant contribution and is expressed in the SVEA as:

$$
\mathbf{P}_{d}(\mathbf{r}, t)=\frac{1}{2} \hat{x}\left[P_{d}(\mathbf{r}, t) \exp \left(-i \omega_{0} t\right)+c . c .\right]
$$

where the slowly varying part $P_{d}(\mathbf{r}, t)$ results from solving the Bloch equations [40]:

$$
\begin{aligned}
\frac{\partial P}{\partial t} & =-\frac{P}{T_{2}}-i\left(\omega_{a}-\omega_{0}\right) P-\frac{i \mu^{2}}{\hbar} E W \\
\frac{\partial W}{\partial t} & =\frac{W_{0}-W}{T_{1}}+\frac{1}{\hbar} \operatorname{Im}\left(E^{*} P\right)
\end{aligned}
$$

where $E$ and $P$ denote the SVEA of both electric field and induced polarization due to dopants respectively. The term $\omega_{a}$ is the atomic transition frequency, $\mu$ is the dipole momentum, $\hbar$ is Planck's constant over $2 \pi$ and the population inversion density $W=N_{1}-N_{2}$ has an initial value $W_{0}$. Also $T_{1}$ is the dopant dependent fluorescence time, which is usually in the range $(0.1 \mu s$ to $10 \mathrm{~ms})$ and $T_{2}$ is the dipole relaxation 
time, which is $\sim 0.1 \mathrm{ps}[42]$. The introduction of $\mathbf{P}_{d}$ incorporated the nonlinear effects, as for dispersive effects a dopant susceptibility is defined in the Fourier domain:

$$
\widetilde{P}(\mathbf{r}, \omega)=\varepsilon_{0} \chi_{d}(\mathbf{r}, \omega) \widetilde{E}(\mathbf{r}, \omega)
$$

To obtain an expression for $\chi_{d}$ Eq.(2.42) is transferred to the Fourier domain:

$$
\left(\omega-\omega_{0}\right) \widetilde{P}=-i \frac{\widetilde{P}}{T_{2}}+\left(\omega_{a}-\omega_{0}\right) \widetilde{P}+\frac{\mu^{2} W}{\hbar} \widetilde{E}
$$

then solving for $\widetilde{P}$ in terms of $\widetilde{E}$ we obtain:

$$
\widetilde{P}=\frac{\mu^{2} T_{2} W(\mathbf{r})}{\hbar\left[T_{2}\left(\omega-\omega_{a}\right)+i\right]} \widetilde{E}
$$

Using Eq.(2.44) and Eq.(2.46) the dopant susceptibility $\chi_{d}$ is given by:

$$
\chi_{d}(\mathbf{r}, \omega)=\frac{\sigma W(\mathbf{r}) n_{0} c / \omega_{0}}{\left(\omega-\omega_{a}\right) T_{2}+i}
$$

where $n_{0}$ is the background linear refractive index. The transition cross section $\sigma$ is:

$$
\sigma=\frac{\mu^{2} \omega_{0} T_{2}}{\varepsilon_{0} n_{0} \hbar c}
$$

Following [42], the Helmholtz equation and Eq.(2.28) remain valid provided that $\chi_{d}$ is added to the refractive index perturbation of Eq.(2.31) in the core region:

$$
\Delta n=n_{2}|E|^{2}+\frac{i \widetilde{\alpha}}{2 k_{0}}+\frac{\chi_{d}}{2 n_{0}}
$$

this will affect the $\Delta \beta$ in Eq.(2.30). Also, both $\beta$ and $\Delta \beta$ must be expanded using a Taylor-series around $\omega-\omega_{a}=\left(\omega-\omega_{0}\right)+\left(\omega_{0}-\omega_{a}\right)[40,42]$. However, assuming the atomic transition frequency $\omega_{a}$ equals the centre of the pulse spectrum $\omega_{0}$, the 
derivation of the propagation equation is simplified considerably. The formulation of $\beta$ holds and $\Delta \beta$ can be obtained by substituting Eq.(2.49) in Eq.(2.30) as follows:

$$
\Delta \beta=\frac{i \alpha}{2}+\gamma|A|^{2}+\left(\frac{1}{2}\right)\left[\frac{1}{\left(\omega-\omega_{0}\right) T_{2}+i}\right] \frac{\sigma \iint_{-\infty}^{\infty} W(\mathbf{r}, t)|F(x, y)|^{2} d x d y}{\iint_{-\infty}^{\infty}|F(x, y)|^{2} d x d y}
$$

It is useful to define a gain $g_{0}$ along the $z$ axis as [40]:

$$
g_{0}(z, t)=\frac{\sigma \iint_{-\infty}^{\infty} W(\mathbf{r}, t)|F(x, y)|^{2} d x d y}{\iint_{-\infty}^{\infty}|F(x, y)|^{2} d x d y}
$$

thus $\Delta \beta$ can be rewritten in a simpler format using Eq.(2.51) as:

$$
\Delta \beta=\frac{i \alpha}{2}+\gamma|A|^{2}+\left(\frac{g_{0}}{2}\right)\left[\frac{1}{\left(\omega-\omega_{0}\right) T_{2}+i}\right]
$$

It is clear that $\Delta \beta$ has become frequency dependent after introducing dopant susceptibility $\chi_{d}$. Using Taylor-series expansion $\Delta \beta$ becomes:

$$
\Delta \beta=\frac{i \alpha}{2}+\gamma|A|^{2}+\left(\frac{g_{0}}{2}\right)\left(-i+T_{2}\left(\omega-\omega_{0}\right)+i T_{2}^{2}\left(\omega-\omega_{0}\right)^{2}+\ldots\right)
$$

and truncating the previous equation at the cubic term as done earlier (neglecting third order dispersion), $\Delta \beta$ can now be substituted in Eq.(2.34) obtaining:

$$
\frac{\partial \widetilde{A}}{\partial z}=\left[i\left(\omega-\omega_{0}\right)\left(\beta_{1}+\frac{g_{0} T_{2}}{2}\right)+\frac{i}{2}\left(\omega-\omega_{0}\right)^{2}\left(\beta_{2}+i g_{0} T_{2}^{2}\right)-\frac{\alpha}{2}+i \gamma|A|^{2}+\frac{g_{0}}{2}\right] \widetilde{A}
$$

at this stage it is possible to use inverse Fourier transform on Eq.(2.54), while replacing $\left(\omega-\omega_{0}\right)$ with $i(\partial / \partial t)$ and $\left(\omega-\omega_{0}\right)^{2}$ with $-\left(\partial^{2} / \partial t^{2}\right)$. The resulting equation is:

$$
\frac{\partial A}{\partial z}=-\beta_{1}^{\mathrm{eff}} \frac{\partial A}{\partial t}-\frac{i}{2} \beta_{2}^{\mathrm{eff}} \frac{\partial^{2} A}{\partial t^{2}}-\frac{\alpha}{2} A+i \gamma|A|^{2} A+\frac{g_{0}}{2} A
$$

with the two effective propagation constants $\beta_{1}^{\text {eff }}=\beta_{1}+g_{0} T_{2} / 2$ and $\beta_{2}^{\text {eff }}=\beta_{2}+i g_{0} T_{2}^{2}$. 
It is worth noting that both $\beta_{1}^{\text {eff }}$ and $\beta_{2}^{\text {eff }}$ are complex and vary along the $z$ axis. The fiber group velocity $v_{g}=1 / \beta_{1}^{\text {eff }}$ is changed due to the dopants, but in practice this affect is negligible [40]. However, this is not the case for $\beta_{2}$, also known as the group velocity dispersion (GVD), as an imaginary part $i g_{0} T_{2}^{2}$ is added due to the parabolic gain. This is physically related to the fiber gain bandwidth [42]. Eq.(2.55) is in the form of a generalized NLSE, with the pulse envelope moving with a group velocity $v_{g}$. For the purposes of investigating mode-locked lasers, it useful to define a frame of reference for the mode-locked pulse (local time) moving at $v_{g}[16]$. This can be done through the following standard Galilean transformation:

$$
T=t-\beta_{1}^{\mathrm{eff}} z
$$

The generalized NLSE can thus be expressed in the form of a CGLE:

$$
i \frac{\partial A}{\partial z}+i \frac{\alpha}{2} A-\frac{1}{2} \beta_{2} \frac{\partial^{2} A}{\partial T^{2}}=i \frac{g_{0}}{2}\left(1+T_{2}^{2} \frac{\partial^{2}}{\partial T^{2}}\right) A-\gamma|A|^{2} A
$$

The gain provided by the EDFA given in Eq.(2.51) can not be evaluated exactly as it depends on $x, y, z$ and the mode profile $|F(x, y)|^{2}$. However, assuming both mode and dopant intensities are uniform over the doped region and that spontaneous emission does not occur over the pulse duration ( $T_{1}$ is longer than pulse width) the saturable gain model can be found using Eq.(2.43) and is expressed as [43]:

$$
G(z)=\frac{g_{0}}{1+\frac{\|A(z)\|^{2}}{E_{s a t}}}
$$

where $\|A(z)\|^{2}=\int_{-\infty}^{\infty}|A(z, T)|^{2} d T$ denotes the pulse energy and $E_{s a t}$ is the saturation energy. In this investigation the saturated gain model adapted in Eq.(2.58) is that presented in $[32,43]$. It is also useful to define the full width at half maximum 
(FWHM) of the fiber amplifier $\Omega$, which is inversely proportional to the dipole relaxation time [42], and can also be expressed in terms of a wavelength spread $\Delta \lambda$ [32]:

$$
\Omega=\frac{1}{T_{2}}=\frac{2 \pi c \Delta \lambda}{\lambda_{0}^{2}}
$$

Nonlinear pulse propagation in a gain saturable EDFA is governed by Eq.(2.57) and Eq.(2.58), which must be solved together in a self-consistant manner. The following section presents the normalization and parameters of the CGLE.

\subsubsection{Fiber Parameters}

An interesting consequence of fiber nonlinearities is optical solitons, which can be supported when there is anomalous dispersion $\left(\beta_{2}<0\right)$. Solitons in optical fiber are nonlinear pulses that propagate nearly dispersion-free for long distances and undergo elastic collisions [44]. Solitons maintain their shape as they propagate due to the interplay between dispersion and nonlinearity. It is known that the NLSE has soliton solutions [16]. Nonlinear pulse propagation in fiber amplifiers is governed by the CGLE, which does not only balance dispersion and nonlinearity, but also loss and gain.

As a consequence, the CGLE is not integrable and does not support solitons from a strictly mathematical point of view [42]. However, it does support solitary-waves, which also maintain their shape on propagation $[25,42,45]$. Thus, it is convenient to normalize the CGLE with dimensionless soliton units as conventionally done with the NLSE [16]. Introducing the following normalized variables [32,42]:

$$
U=\frac{A}{\sqrt{P_{0}}} \quad \xi=\frac{z}{L_{D}} \quad \tau=\frac{T}{T_{0} / 1.76}
$$

where $P_{0}$ is the field peak power, $T_{0}$ is the FWHM of an arbitrary reference pulse width and $L_{D}=\left(T_{0} / 1.76\right)^{2} /\left|\beta_{2}\right|$ denotes the dispersion length. In addition the following 
normalized constant definitions are adapted for simplification:

$$
\begin{aligned}
N^{2} & =\frac{\gamma P_{0}}{\left|\beta_{2}\right|}\left(\frac{T_{0}}{1.76}\right)^{2} \\
\Gamma & =\frac{\alpha}{2} L_{D} \\
G_{0} & =\frac{g_{0}}{2} L_{D} \\
\tau_{\Omega} & =\frac{1}{\Omega^{2}}\left(\frac{1.76}{T_{0}}\right)^{2}
\end{aligned}
$$

thus the CGLE can now be expressed in a normalized form as $[32,46]$ :

$$
i \frac{\partial U}{\partial \xi}+i \Gamma U-\frac{1}{2} \operatorname{sgn}\left(\beta_{2}\right) \frac{\partial^{2} U}{\partial \tau^{2}}=i G(\xi)\left(1+\tau_{\Omega} \frac{\partial^{2}}{\partial \tau^{2}}\right) U-N^{2}|U|^{2} U
$$

where the SVEA of the electric field $U$, with the amplitude normalized by the peak field power $P_{0}$, propagates through fiber with loss $\Gamma$, second order dispersion $\beta_{2}$ and a nonlinear parameter $\gamma$. The variable $\tau$ denotes the local time in the rest frame of the mode-locked pulse normalized by $T_{0} / 1.76[35,47]$. The variable $\xi$ is the distance the pulse travels in the cavity normalized by the dispersion length $L_{D}$. The term $\operatorname{sgn}\left(\beta_{2}\right)=+1$ or -1 depending on whether $\beta_{2}$ is positive or negative. In general $\beta_{2}$ is negative to support soliton solutions. The parameter $N$ is the soliton order of the corresponding NLSE $[16,32]$. For undoped fibers $G(\xi)=0$, however, for the EDFA used in this model, the saturable gain follows Eq.(2.58) and is given by [2]:

$$
G(\xi)=\frac{2 G_{0}}{1+\frac{\|U(\xi)\|^{2}}{E_{\text {sat }}}}
$$

where $G_{0}$ is the normalized saturated gain (due to laser pump) given in Eq.(2.63). The term $E_{\text {sat }}$ denotes the normalized saturation energy and $\tau_{\Omega}=\left(1 / \Omega^{2}\right)\left(1.76 / T_{0}\right)^{2}$ is the normalized gain bandwidth where $\Omega$ denotes the FWHM bandwidth of the laser gain given by Eq.(2.59). The expression $\|U(\xi)\|^{2}=\int_{-\infty}^{\infty}|U(\xi, \tau)|^{2} d \tau$ is the normalized 
energy of the current mode-locked pulse. It is worth noting that $\tau_{\Omega}$ is the parameter that controls the resulting pulse width of the mode-locked pulse, as it governs the gain bandwidth of the EDFA $[2,9,47]$. Based on this normalization the single-soliton solution of the corresponding NLSE has a peak power given by:

$$
P_{0}=\frac{\lambda_{0} A_{e f f}}{2 \pi n_{2} L_{D}}
$$

where the wavelength of operation is $\lambda_{0}=1550 \mathrm{~nm}$, the effective cross-sectional area of the fiber is $A_{\text {eff }}=60 \mu \mathrm{m}^{2}$ and the nonlinear refractive index is given by $n_{2}=2.6 \times 10^{-16} \mathrm{~cm}^{2} / \mathrm{W}[32]$. The average cavity dispersion is assumed to be anomalous to support solitary-wave solutions $D=-\left(2 \pi c / \lambda_{0}^{2}\right) \beta_{2}=12 \mathrm{ps} / \mathrm{km} . \mathrm{nm}$ [47]. The parameter $N$ is normalized to 1 for fundamental soliton-like formation [16]. Using the above parameters and definitions and assuming the FWHM of the reference pulse width $T_{0}=200 \mathrm{fs}$, the dispersion length is calculated to be $L_{D}=0.84370 \mathrm{~m}$, thus resulting in the dimensionless parameter $\Gamma=1.94 \times 10^{-5}$ for an attenuation of 0.2 $\mathrm{dB} / \mathrm{km}$ and $G_{0}=1-4.5$ for an amplification range of 5-26 dB which is physically realizable in a $10 \mathrm{~m}$ EDFA pumped at $1.48 \mu \mathrm{m}[1,48]$. The normalized gain parameters in Eq.(2.66) follow those in $[32,47]$, with the dimensionless term $\tau_{\Omega}=0.1$ corresponding to a gain bandwidth of $25 \mathrm{~nm}$ and $E_{\text {sat }}=1$. The normalized EDFA length $\xi=1$.

It is worth noting that the fiber section of the laser model in Fig. 2.1 modeled by Eq.(2.65) is an approximation of three connected fiber segments, with only one being an EDFA. The length of the fiber is $1-10 \mathrm{~m}$ with a $10-30 \mathrm{~cm}$ of EDFA. A more accurate model would involve propagating the laser pulse through three different segments, two of which are modeled using the conventional NLSE and the other modeled using Eqs. (2.65) and (2.66). Although the different dispersion and effective cross-sectional areas between EDFA and undoped fibers [32] can cause errors, numerical results indicate incorporating the effect of all three fiber sections is an accurate approximation [49]. 


\subsection{LPFG Equations}

This section introduces the nonlinear mode coupling dynamics of the LPFG and its governing equations. A LPFG is a fiber optical structure with a periodically varying refractive index. The period of a LPFG is usually in the order of a millimeter, which considerably exceeds the wavelength of light in the fiber. As a consequence of the large period, LPFGs are easily manufactured through ultraviolet radiation or even mechanical means [50]. The LPFG couples two copropagating modes [12]. When considering a single mode optical fiber, the LPFG couples the fundamental core mode to higher-order modes propagating in the cladding [35,51,52]. The choice of the grating period can be made so a phase matching condition for copropagating modes is satisfied. Analytically, the phase matching condition can be expressed as:

$$
\beta_{\text {core }}-\beta_{\text {clad }}^{n}=\frac{2 \pi}{\Lambda}
$$

where $\Lambda$ is the grating period, $\beta_{\text {core }}$ and $\beta_{\text {clad }}^{n}$ denote the propagation constant of the core and $n^{\text {th }}$ cladding mode. A schematic of a LPFG with phase matching between the guided core mode and forward propagating cladding mode is shown in Fig. 2.3(a). The transmission spectrum of such gratings exhibit dips at wavelengths corresponding to resonances with different cladding modes [51]. For example, Fig. 2.3 (b) shows a transmission spectrum (in decibels) calculated for a typical LPFG. The simulation was performed using OptiGrating as part of the Optiwave software package [53]. The grating is written in a standard single mode fiber with a core and cladding radius of 4 $\mu m$ and $75 \mu m$ respectively. Further, the refractive index is assumed to be 1.48 in the core section and 1.475 in the cladding section. The length of the grating is set to 25 $\mathrm{mm}$, with a peak induced index change of $3.6 \times 10^{-4}$ and a uniform profile. The grating period is $579 \mu \mathrm{m}$ and was chosen to yield coupling at $1550 \mathrm{~nm}$ between the core 


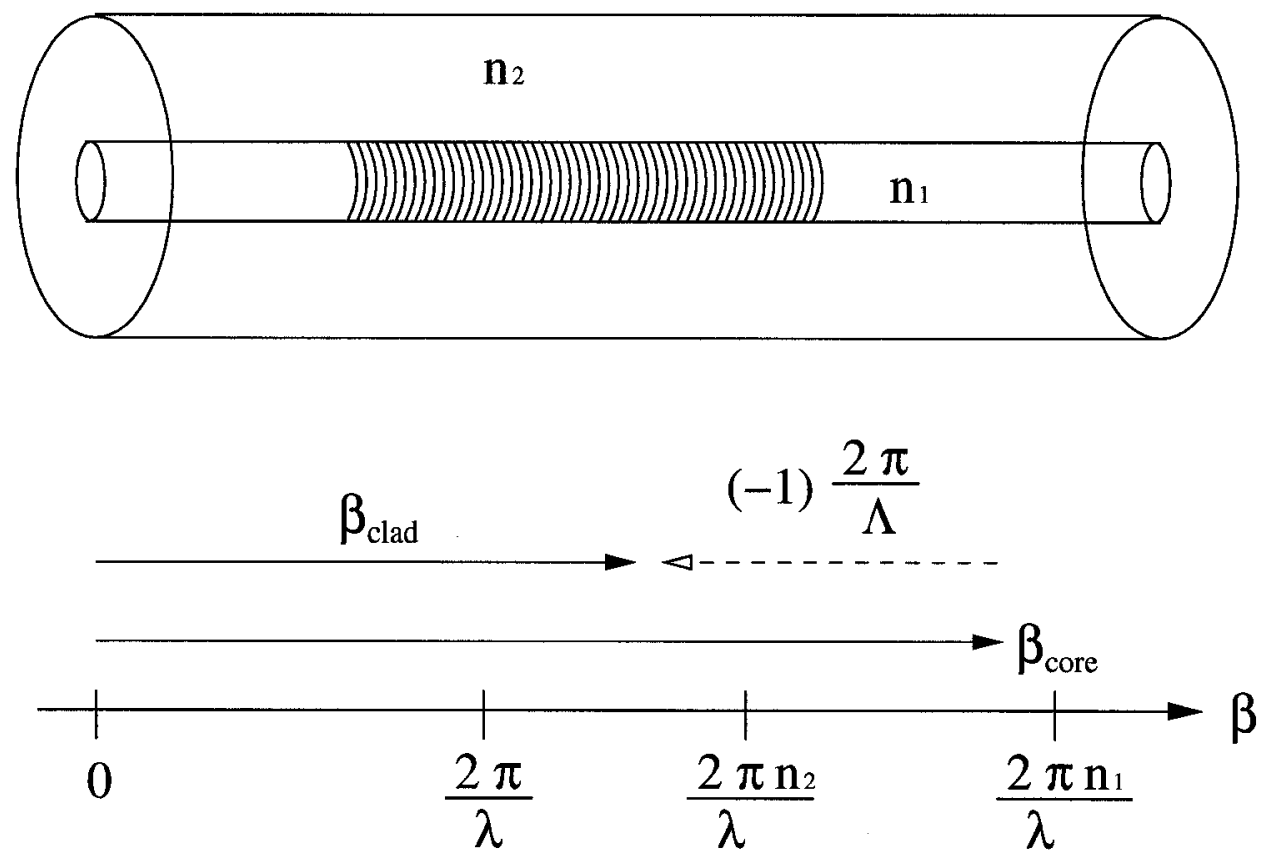

(a)

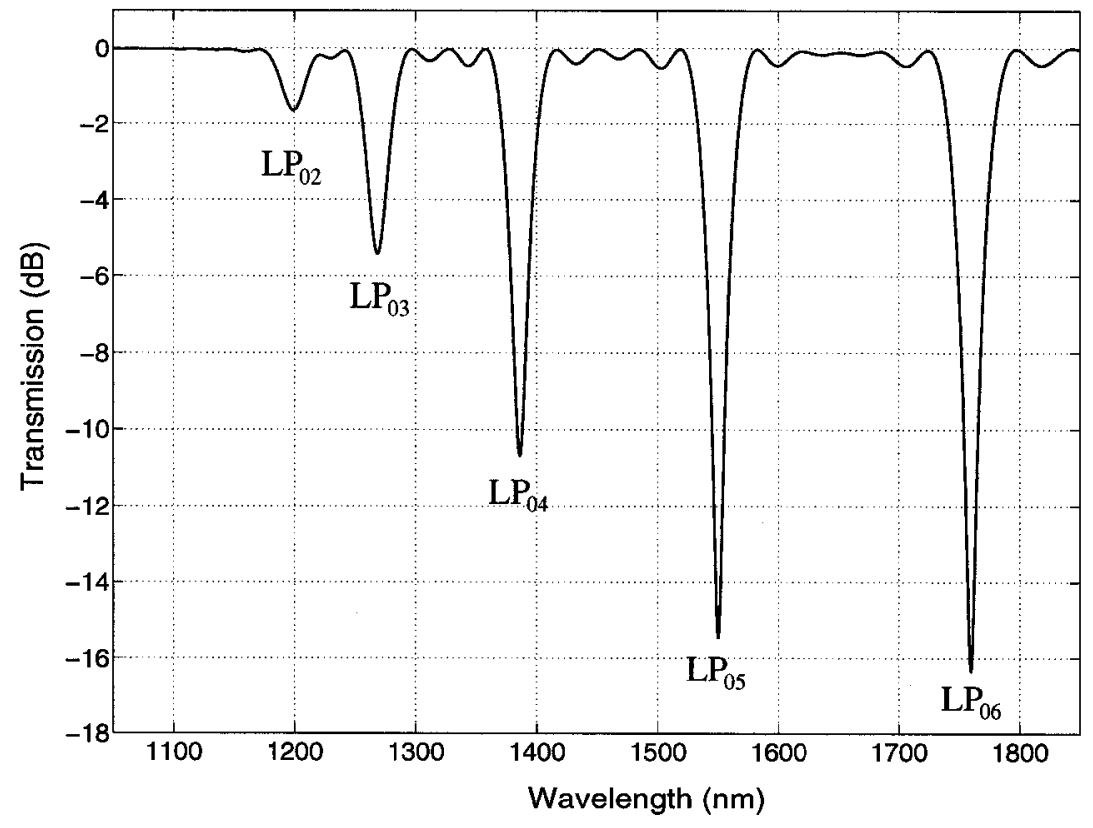

(b)

Figure 2.3: Coupling of the core mode into copropagating cladding mode in a 25 mm LPFG written in a single mode fiber (a) schematic. (b) transmission spectrum. 
mode and the $5^{\text {th }}$ copropagating cladding mode. The simulation only considered coupling between linearly polarized (LP) modes. The dips appearing in the transmission spectrum of Fig. 2.3 (b) correspond to resonances between core and cladding modes of order $2,3,4,5$ and 6 . Normally, energy is transferred periodically between the core and cladding modes of a LPFG as a result of the resonant and linear mode-coupling interaction [12]. However, introducing nonlinearity in the LPFG shifts the propagation constant of each mode via self-phase and cross-phase modulation and hence detunes the mode-coupling interaction [54]. Thus, by carefully choosing the grating period, a resonant coupling between the copropagating core and cladding modes can be achieved, causing the low intensity portions of the pulse to be transferred to the cladding and attenuated, while the high intensity portion is detuned from resonance by nonlinearity [32]. This pulse-shaping introduced by the nonlinear mode-coupling dynamics of the LPFG is the principle mode-locking mechanism.

\subsubsection{Nonlinear Mode Coupling}

Nonlinear optical devices usually exploit second order $\chi^{(2)}$ or third order $\chi^{(3)}$ nonlinearities. However, due to the inversion symmetry imposed in optical fibers the effect of $\chi^{(2)}$ is eliminated. Consequently, nonlinear fiber devices utilize the much weaker third order nonlinearity, which result in an intensity dependent refractive index, such as LPFGs written in highly nonlinear fibers. The nonlinear coupled mode equations of the grating is asymptotically derived from Maxwell's equations in [35]. The derivation is similar to that of the NLSE presented in [16], with the same quasi-monochromatic wave approximation. In addition, a paraxial and rotating wave approximations are required and justified [35]. The derivation starts with the wave equation, with the 


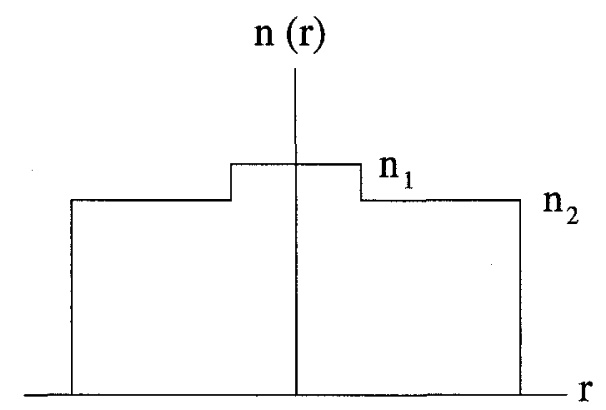

Figure 2.4: Transverse refractive index profile for a single mode fiber with core refractive index $n_{1}$ and cladding refractive index $n_{2}$.

index of refraction modified to account for the grating's periodicity as [35]:

$$
n(x, y, z)=n_{0}(x, y)\left[1+\Delta n_{\mathrm{UV}} \cos \left(\frac{2 \pi}{\Lambda} Z\right)\right]
$$

where $n_{0}(x, y)$ accounts for the transverse index change as shown in Fig. 2.4 and $\Delta n_{\mathrm{UV}}$ is the ultraviolet-induced index change in the longitudinal direction, which is usually in the order of $10^{-4}$. Following [35], it is convenient to assume a single dominant Fourier mode component for a sinusoidal grating periodicity. Further, we assume a single mode fiber, with the LPFG coupling the fundamental core mode to higher order cladding modes. The strength of this coupling was found to depend on the order of the cladding mode [51]. The structure of the guided modes of the fiber can be calculated from the eigenfunctions of Eq.(2.27). These solutions can be expressed in terms of LP modes $\phi_{n m}$ where $n, m$ are the azimuthal and radial mode numbers respectively [12]. Here, we consider only coupling between radially symmetric modes with $m=0$ [35]. Fig. 2.5 shows the normalized eigenfunctions of the first fundamental core mode and the first three radially symmetric cladding modes. The eigenfunctions are normalized according to [32]:

$$
\left(\phi_{n}, \phi_{n}\right)_{r}=\int_{0}^{\infty} \phi_{n} \phi_{n} r d r=1
$$



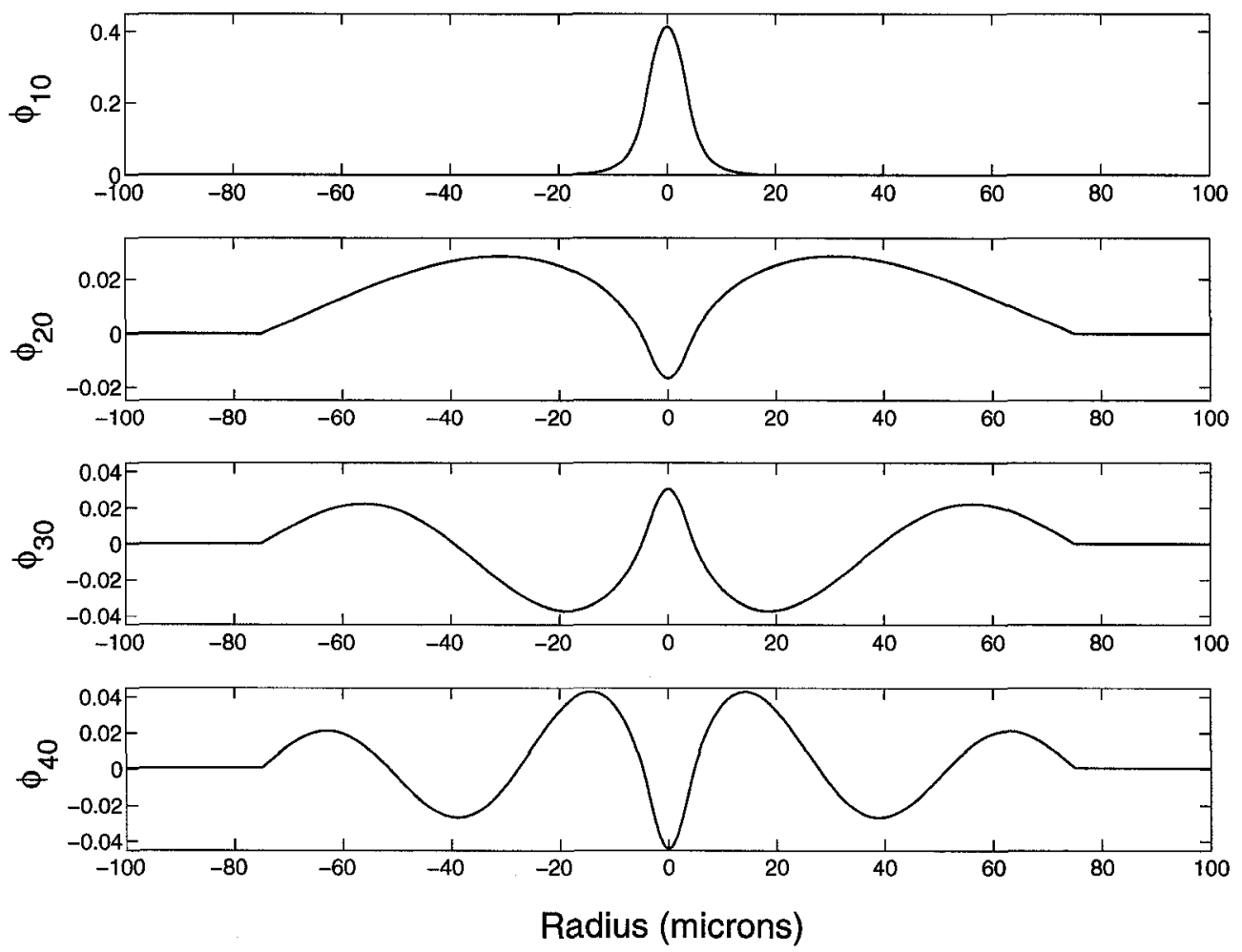

Figure 2.5: Normalized modal profile of the radially symmetric modes $(m=0)$. The fundamental core mode is denoted $\phi_{10}$, while the cladding modes are denoted $\phi_{2-4,0}$.

The solutions in Fig. 2.5 were obtained by the commercial LP mode solver provided by OptiGrating as part of the Optiwave software package [53]. The modal profiles are used in calculating coupling coefficients, which in turn are used in the nonlinear mode coupling equations. The profiles in Fig. 2.5 are obtained for a LPFG written in a standard single mode fiber SMF-28 [55]. The length of the LPFG was set to $50 \mathrm{~mm}$, while the core and cladding radius was assumed to be $4 \mu \mathrm{m}$ and $75 \mu \mathrm{m}$ respectively. The core index is $n_{1}=1.48$ and the cladding index $n_{2}=1.475$ resulting in a refractive index difference of $0.34 \%$ and only one core mode supported $\phi_{10}$ at a wavelength of $1550 \mathrm{~nm}$. Also, the depth of the induced index modulation was set to $\Delta n_{\mathrm{UV}}=8 \times 10^{-4}[32,35]$. 
The equations governing the nonlinear coupling between the copropagating core $(i=1)$ and cladding $(j)$ modes are derived by Kutz et al. $[32,35]$ :

$$
\begin{aligned}
& i \frac{\partial U}{\partial \xi_{G}}+\left(c_{11}|U|^{2}+2 c_{1 j}|V|^{2}\right) U+d_{1 j} V-\frac{\Delta}{2} U=0 \\
& i \frac{\partial V}{\partial \xi_{G}}+\left(c_{j j}|V|^{2}+2 c_{1 j}|U|^{2}\right) V+d_{1 j} U+\frac{\Delta}{2} V=0
\end{aligned}
$$

where $U$ and $V$ are the SVEA of the electric field in the core and cladding respectively, $c_{i j}$ and $d_{i j}$ denote the linear and nonlinear coupling coefficients respectively and the parameter $\Delta$ measures the detuning from the resonant coupling [32,35]. Using the amplitude normalization in Eq.(2.65), the normalized coupling coefficients are:

$$
\begin{gathered}
c_{1 j}=\frac{4 \pi n_{2}^{g}}{\lambda_{0} A_{e f f}} \frac{\left(\left|\phi_{10}\right|^{2},\left|\phi_{j 0}\right|^{2}\right)_{r}}{\left(\left|\phi_{10}\right|^{2},\left|\phi_{10}\right|^{2}\right)_{r}} L_{D} P_{0} \\
d_{1 j}=\frac{\Delta n_{\mathrm{UV}} \pi}{2 \lambda_{0}}\left(n_{0}^{2}(r) \phi_{10}, \phi_{j 0}\right)_{r} L_{D}
\end{gathered}
$$

with $n_{2}^{g}$ being the nonlinear refractive index of the LPFG section, $(A, B)_{r}$ is defined in Eq. (2.70), $n_{0}(r)$ is shown in Fig. 2.4 and the resonant detuning parameter $\Delta$ is [35]:

$$
\Delta=\left[\left(\frac{2 \pi}{\Lambda}\right)-\frac{\left(\beta_{1}-\beta_{j}\right)}{2 k}\right] L_{D}
$$

In Eqs.(2.71) and (2.72) the effect of second order dispersion has been neglected. The coefficient $d_{1 j}$ is proportional to the ultraviolet index modulation, which directly affects the linear coupling strength. The nonlinear coefficients $c_{j j}$ and $c_{1 j}$ measure self-phase and cross-phase modulation respectively [35]. The analysis of nonlinear pulse propagation in the LPFG assumes no difference in the group velocity dispersion between the core and cladding; this is justified provided the LPFG is short. In contrast, for longer LPFG the group velocity mismatch can cause temporal walkoffs [35]. Further, the ultraviolet index modulation is assumed to preserve radial 
symmetry [35]. The radially dependent intensity is incorporated in the coefficients $c_{i j}$ and $d_{i j}$. Further, when $c_{11}=c_{j j}$ the analysis is similar to the nonlinear coherent coupler [54]. Although nonlinearity does not allow complete periodic energy transfer from core to cladding, it allows partial intensity dependent and nonperiodic energy transfer causing intensity discrimination. In this case, the low intensity linear parts of the pulse resonates with the cladding mode, while the high intensity peak field is detuned from resonance and transmitted efficiently. As the coupling coefficients do not only depend on the overlap integral but also on the normalized LPFG length and nonlinear refractive index ration $n_{2}^{g} / n_{2}$. Hence, obtaining $c_{11}=c_{j j}$ can be done through material choice and appropriate mode selection based on the grating period.

Following the work in $[32,35]$, we will only consider coupling between the core mode and the third cladding mode $n=4$. In principle, coupling could be between the core mode and any cladding mode. However, this will result in different modal profiles, overlap integrals and coupling coefficients. This is illustrated in Fig. 2.6 where the values of the linear and nonlinear coupling coefficients as a function of the $n^{\text {th }}$ radially symmetric cladding mode are plotted. The linear coupling response is maximum at $n=10$, while for $n=20$ the linear coupling is minimized relative to the nonlinear coupling strength. For pulse shaping it is desirable to maximize the ratio of the nonlinear coupling coefficients to the linear coefficient allowing more flexibility in LPFG design. However, coupling to higher order cladding modes increases the value of $n_{2}^{g}$ to hundreds of times that of conventional single mode fibers.

\subsubsection{Pulse Shaping}

The use of a LPFG for nonlinear optical switching and pulse reshaping was first reported in $[35,56]$. Through introducing nonlinearity in the LPFG and by carefully choosing the grating period, a resonant coupling between the copropagating core and cladding modes can be achieved, causing the low intensity portions of the pulse to be 


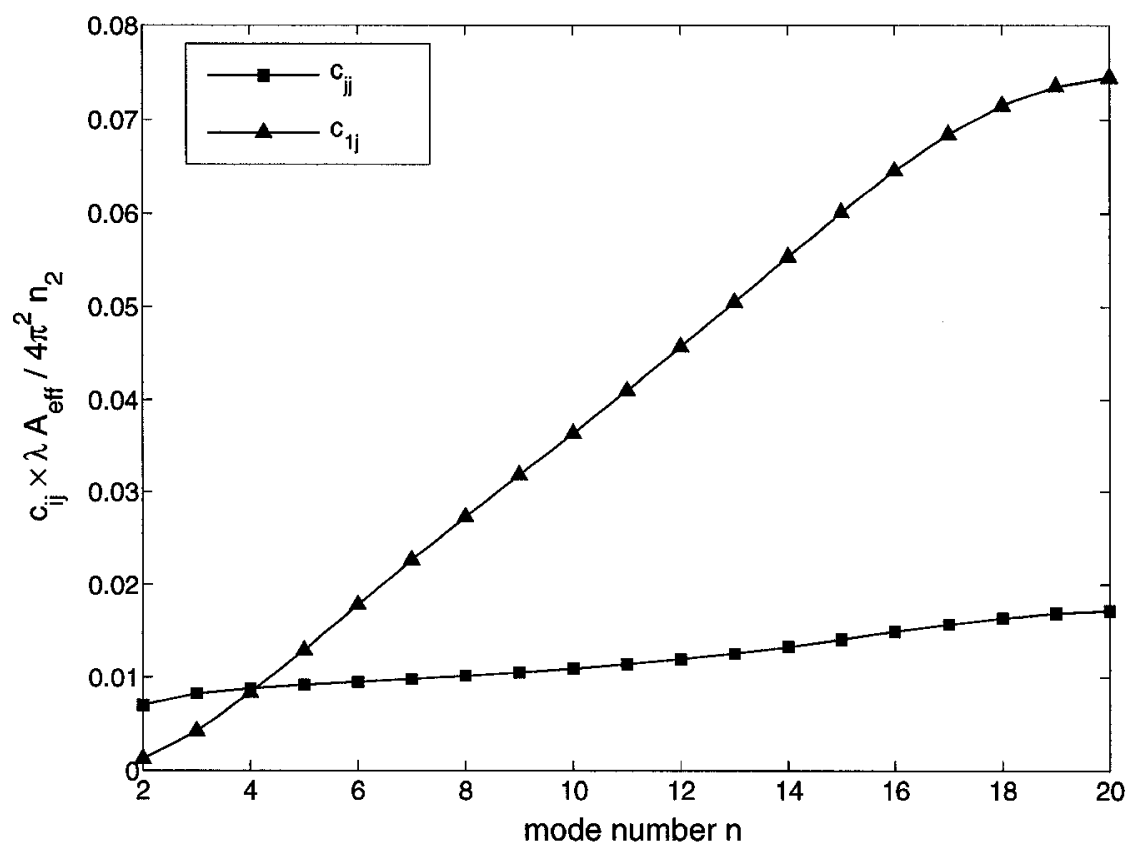

(a)

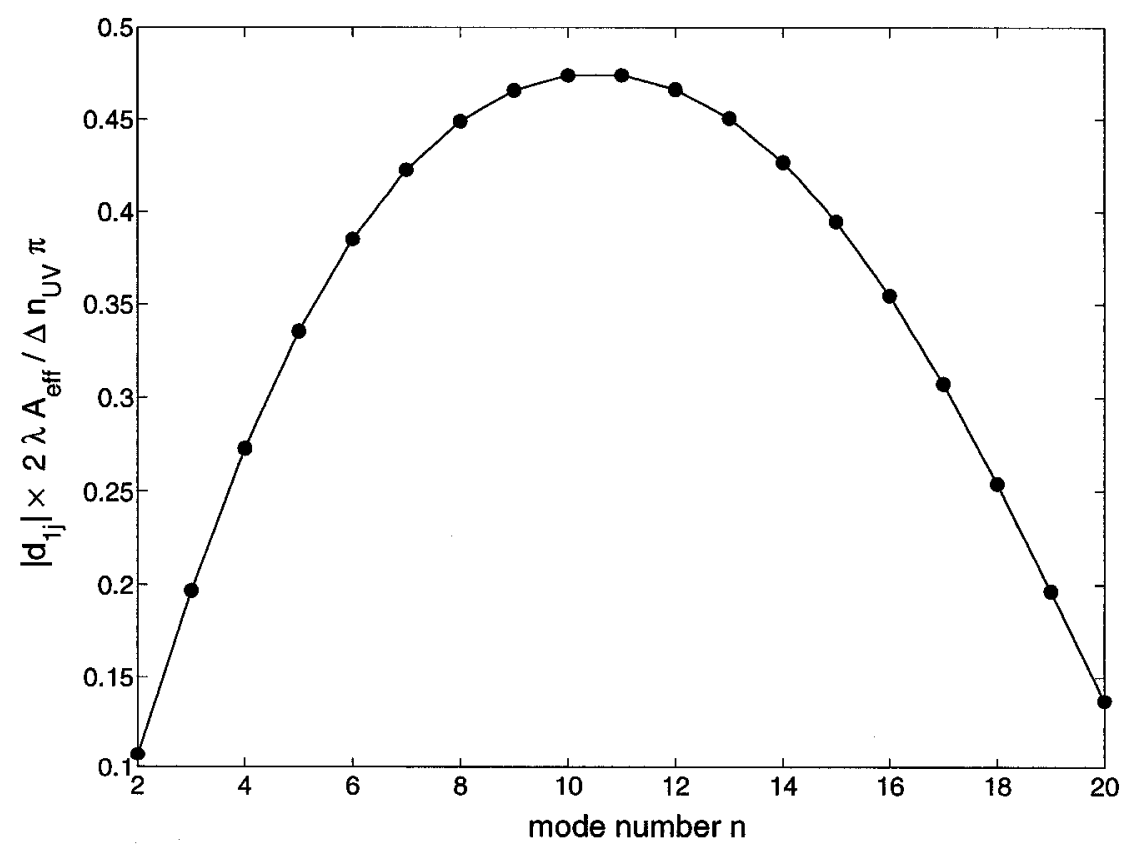

(b)

Figure 2.6: LPFG coupling coefficients as a function of the $n^{\text {th }}$ radially symmetric cladding mode for $j=1-20$ (a) linear $d_{1 j}$. (b) nonlinear $c_{1 j}$ and $c_{j j}$. 
transferred to the cladding and attenuated resulting in the appearance of resonance loss in the transmission spectrum, while the high intensity portion is detuned from resonance by nonlinearity. This process forms an intensity discrimination, through which a stable mode-locked pulse is attained. The pulse shaping mechanism of the LPFG can be demonstrated through simulating Eqs.(2.71) and (2.71) using MATLAB [33] (see Chapter 3). To illustrate pulse shaping, following the numerical investigation by Kutz et al. [32,35], a LPFG is assumed to have a normalized length of $\xi_{G}=0.7$, corresponding to a physical length of $60 \mathrm{~cm}$ with coupling occurring between the core mode and third order cladding mode. The radius of the core and cladding are $4 \mu \mathrm{m}$ and $75 \mu \mathrm{m}$ respectively. The core index is $n_{1}=1.486$, while the cladding index $n_{2}=1.45$. The normalized coupling coefficients are $d_{14}=2, c_{11}=c_{44}=0.3$, $c_{14}=0.5$ and $\Delta=0$ for resonant coupling [35]. Fig. 2.7 shows the initial and final profile resulting from propagating a Gaussian pulse with an amplitude of 4 units in the core through the LPFG. The cladding is assumed to be cleared initially i.e. no light is launched into the cladding. The profile evolution of the same Gaussian pulse as it propagates down the grating length is shown in Fig. 2.8. The results show pulse shaping, while energy is exchanged to the cladding. However, the pulse peak value is maintained. The results reported in Fig. 2.8 and 2.7 are in agreement with the experimental data obtained in [35]. However, the work done in [57] provided better agreement with Kutz's experimental results, as it analyzed nonlinear propagation in the LPFG using discrete coupled mode theory.

To efficiently couple the core mode to the third order cladding mode a nonlinear refractive index within the LPFG is about 20 times larger than conventional optical fiber. This high nonlinear index of refraction problem can be solved through writing the LPFG in a photonic crystal fiber (PCF) or chalcogenide fiber. PCFs confine light through the photonic bandgap mechanism and can operate single mode at a wide range of wavelengths with tailorable dispersion, nonlinearity and large mode 


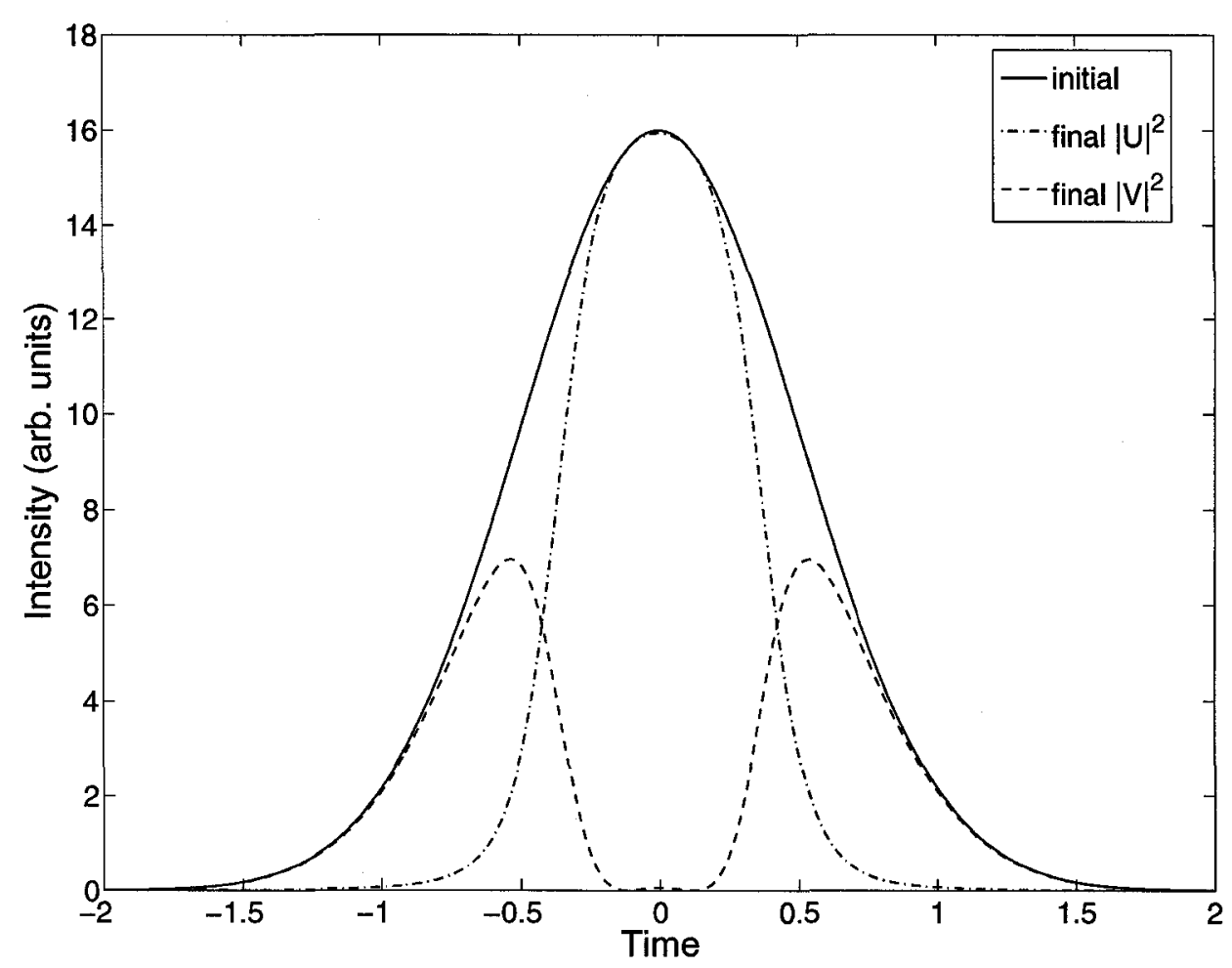

Figure 2.7: Initial and final pulse profile resulting from propagating a Gaussian pulse in a LPFG. The simulation parameters are $d_{14}=2, c_{11}=c_{44}=0.3, c_{14}=0.5$, $\Delta=0$ and $\xi_{G}=0.7$ within a computational domain of $T \in[-5,5]$.

field diameter [58]. Highly nonlinear PCFs have recently been employed in laser optics [59]. Further, fabrication techniques for writing a LPFG in a PCF using a $\mathrm{CO}_{2}$ laser has been reported in [60], while the application of a LPFG in a PCF has been recently used for biochemical sensing [61]. The other viable solution is writing the LPFG in a highly nonlinear $\mathrm{As}_{2} \mathrm{Se}_{3}$ based glass fiber, also known as chalcogenide fiber [62]. These type of fibers were reported to have the highest Kerr nonlinearity $n_{2}$ relative to other types of fiber. The use of chalcogenide fiber in all optical switching has been reported in [62,63], with a nonlinear refractive index $n_{2}$ reaching $200 \times$ conventional silica fibers. Recently, higher nonlinearity has been achieved with $n_{2}$ 


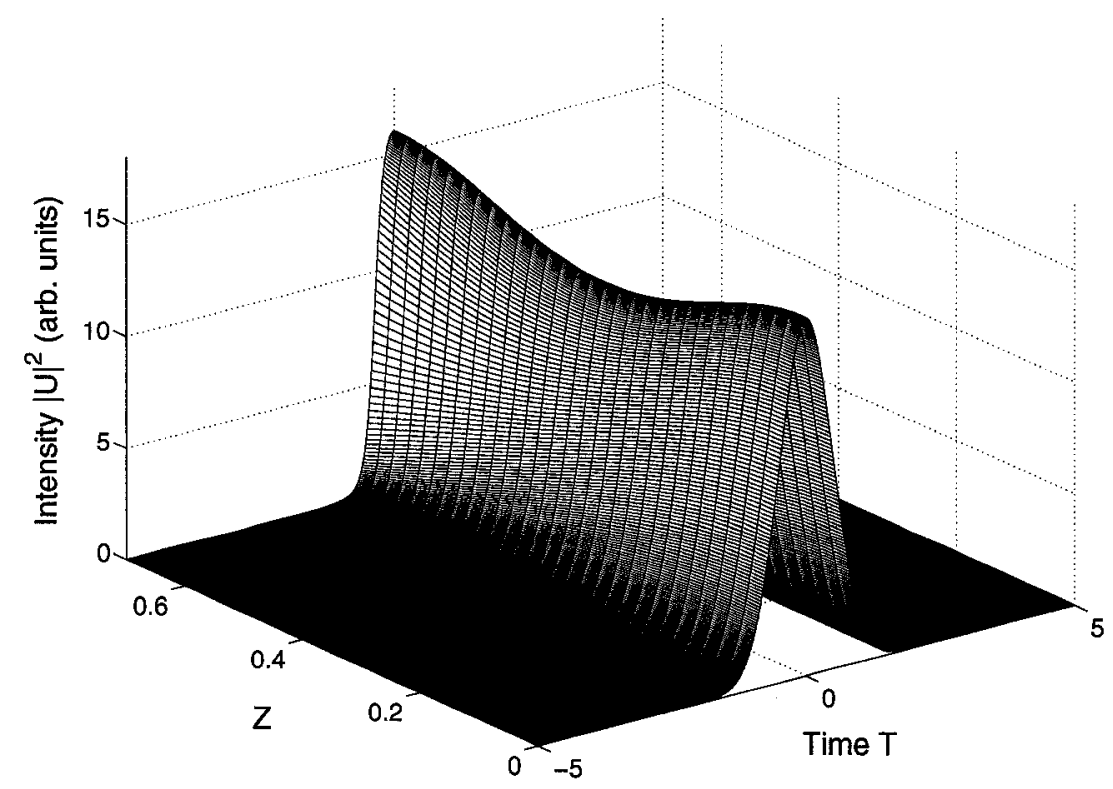

(a)

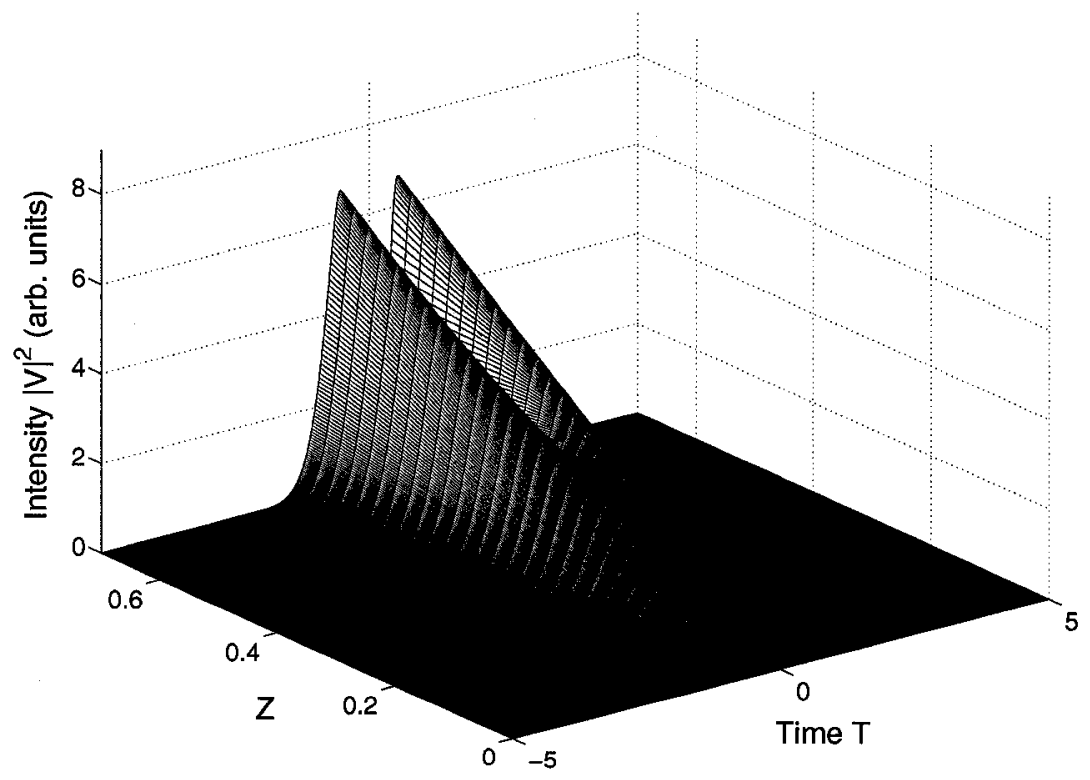

(b)

Figure 2.8: Evolution profile of a Gaussian pulse through a LPFG. The simulation parameters are $d_{14}=2, c_{11}=c_{44}=0.3, c_{14}=0.5, \Delta=0$ and $\xi_{G}=0.7$ within a computational domain of $T \in[-5,5]$ (a) core profile. (b) cladding profile. 
on the order of $400 \times$ silica [64,65]. Further, the first demonstration of a LPFG written in a single mode chalcogenide fiber has been recently reported in [66], where a threaded rod is pressed against a short $\mathrm{As}_{2} \mathrm{Se}_{3}$ fiber writing a LPFG. 


\section{Chapter 3}

\section{Numerical Methods and Implementation}

Over the past decade considerable effort has been put into solving nonlinear partial differential equations, similar to Eqs.(2.39) and (2.65) numerically and analytically. The inverse scattering transform is currently the standard analytical technique employed in obtaining the soliton solution of the NLSE [67]. However, the analytical methods are only valid for certain cases and we usually resolve to numerical methods for obtaining a full problem solution. The typical numerical methods used in solving these type of equations can be either finite difference method (FDM) [37,68] or split step Fourier method (SSFM) [69]. In this investigation variations of the SSFM will be implemented rather than FDM as it is orders of magnitude slower while achieving the same accuracy [70]. This is due to the availability of highly efficient fast-Fouriertransform (FFT) algorithm implementations, which are employed extensively by any SSFM. In this chapter, the basic SSFM is introduced before presenting two different variations, specifically, the symmetric and predictor-corrector SSFM. The need to explore these numerical techniques arise from the wide range of nonlinear dynamics exhibited by this type of laser [46], hence demanding highly robust and numerically stable algorithms. Further, an object oriented programming (OOP) approach is adapted in coding the numerical algorithms used in solving the mode-locked laser model. The merits and demerits of this approach will be discussed. 


\subsection{Split-Step Fourier Method}

The numerical simulation performed is based on solving the fiber and grating equations with variations of the SSFM [16]. Conventionally, the SSFM has been applied to the NLSE $[16,71]$. In this work, the solitary-wave operation of the passively modelocked laser is modeled using the CGLE, with a gain model governed by Eq.(2.66) and the LPFG Eqs.(2.71) and (2.72). In this section, the SSFM is applied to both EDFA and LPFG governing equations.

\subsubsection{Fiber Equation}

The SSFM is based on splitting the CGLE into two separate operators, the difference linear operator $\hat{D}$ that accounts for second order dispersion, absorption and saturable gain in the EDFA, i.e. the linear part of the CGLE, and the nonlinear operator $\hat{N}$ that accounts for the nonlinearity induced during ultrashort pulse propagation. To explain the idea behind this method it is useful to rewrite Eq. (2.65) as [16]:

$$
\frac{\partial U}{\partial \xi}=(\hat{D}+\hat{N}) U
$$

where both linear and nonlinear parts of the CGLE are given by:

$$
\begin{aligned}
& \hat{D}=-\Gamma-\frac{i}{2} \operatorname{sgn}\left(\beta_{2}\right) \frac{\partial^{2}}{\partial \tau^{2}}+G(\xi)+G(\xi) \tau_{\Omega} \frac{\partial^{2}}{\partial \tau^{2}} \\
& \hat{N}=i N^{2}|U|^{2}
\end{aligned}
$$

Usually, both linear and nonlinear effects act together along the propagation distance. However, the SSFM approximation assumes that dispersion, absorption and gain act together, while nonlinearity acts separately over a short fiber distances $h$. When the pulse propagates from $\xi$ to $\xi+h$ two steps are performed, the first step nonlinearity acts alone and $\hat{D}=0$, while the second step linear effects act alone and $\hat{N}=0$, 
mathematically the normalized SVEA of the electric field can be expressed as [16]:

$$
U(\xi+h, \tau)=\exp (h \hat{D}) \exp (h \hat{N}) U(\xi, \tau)
$$

The dispersion operator is computed in the frequency domain, while the nonlinear operator is computed in the time domain. The transition from one domain to another is done numerically using FFT algorithms [72]. Thus Eq. (3.4) can be written as:

$$
U(\xi+h, \tau)=F_{T}^{-1} \exp (h \hat{D}(i \omega)) F_{T} \exp (h \hat{N}) U(\xi, \tau)
$$

with $F_{T}$ and $F_{T}^{-1}$ denoting Fourier transform and its inverse respectively. The calculation of $\hat{D}(i \omega)$ is straightforward through replacing the differential operator $\partial / \partial \tau$ with $i \omega$, where $\omega$ is the frequency in the Fourier domain. The accuracy of the SSFM can be improved by including the effect of nonlinearity in the middle of the propagation segment from $\xi$ to $\xi+h$ rather than at the boundary [16]. Due to the symmetric nature of the exponential operator this method was named symmetric split-step Fourier (S-SSFM). Thus the normalized SVEA can be expressed as [16]:

$$
U(\xi+h, \tau)=\exp \left(\frac{h}{2} \hat{D}\right) \exp \left(\int_{\xi}^{\xi+h} \hat{N}\left(\xi^{\prime}\right) d \xi^{\prime}\right) \exp \left(\frac{h}{2} \hat{D}\right) U(\xi, \tau)
$$

In Eq. (3.6), when the step size is small enough, the integral in the middle section can be approximated by $\exp (h \hat{N})$, similar to Eq. (3.4). However, a more accurate approach would involve implementing a trapezoidal rule for the midpoint integral:

$$
\int_{\xi}^{\xi+h} \hat{N}\left(\xi^{\prime}\right) d \xi^{\prime} \approx \frac{h}{2}[\hat{N}(\xi)+\hat{N}(\xi+h)]
$$

where the integration in Eq. (3.7) is not straightforward as the $\hat{N}(\xi+h)$ is unknown in the midsegment at $\xi+h / 2$. The solution to this problem relies on an iterative 


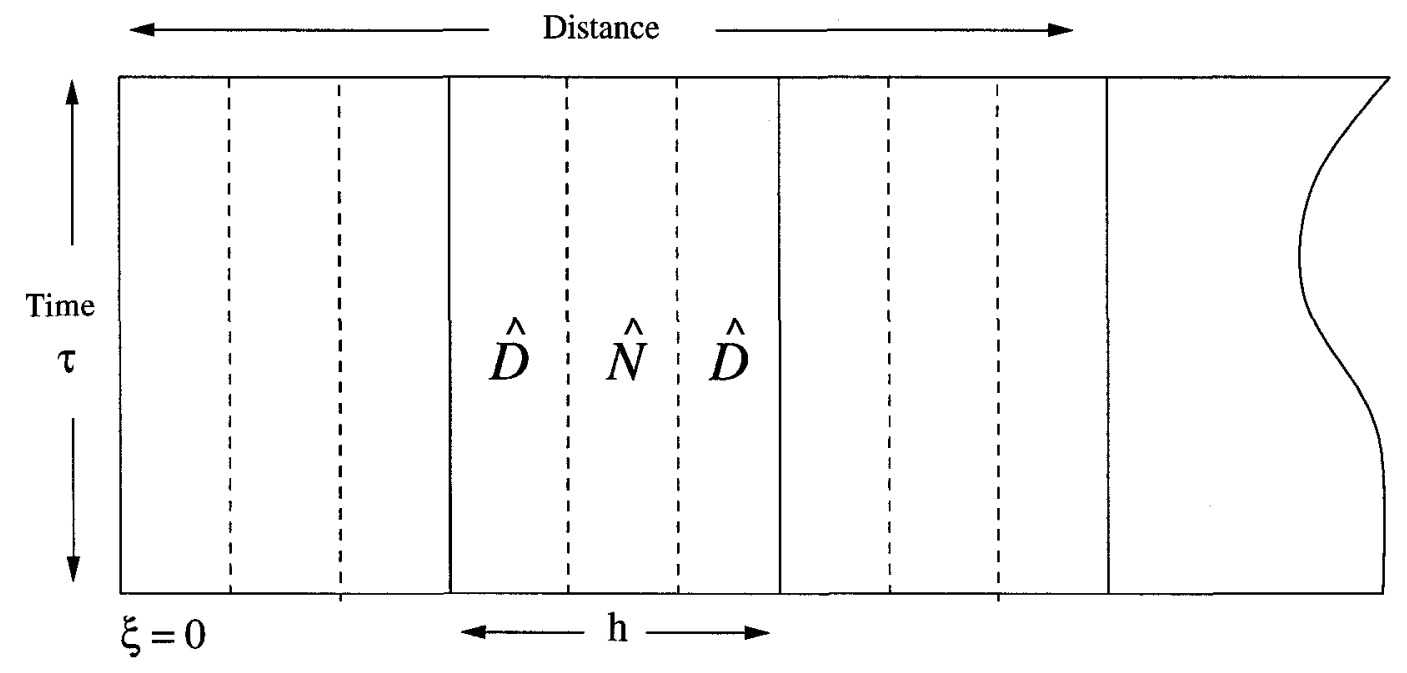

Figure 3.1: Illustration of the S-SSFM. The nonlinear operator $\hat{N}$ is evaluated in the midpoint between the linear operators $\hat{D}$ within the segment $\xi$ to $\xi+h$.

approach which initially starts with replacing $\hat{N}(\xi+h)$ by $\hat{N}(\xi)$. Fig. 3.1 shows a schematic illustrating the S-SSFM. The fiber length is divided into segments of width $h$, while the temporal widow is composed of a number of points. A more detail survey of the variations of optimized SSFM algorithms are reported in [73].

It is clear that SSFM uses functional evaluations at $\xi$ and $\xi+h$. However, it does not retain information for use in future evaluations. If we divide the propagation length into mesh points $j$, and the approximate solution is available at $\xi_{0}, \xi_{1}, \xi_{2} \ldots$ $\xi_{j}$, the accuracy of the approximation at $\xi_{j+1}$ can be improved through utilizing a multistep method including explicit Adams-Bashforth and implicit Adams-Moulton. This combination of explicit and implicit methods is known as the predictor-corrector (PC-SSFM) [71]. First the predicted normalized SVEA can be expressed as:

$$
\begin{array}{r}
\overline{U\left(\xi_{j+1}, \tau\right)}=\exp \left(\frac{h}{2} \hat{D}\right) \exp \left[h \left(A_{j} \hat{N}_{j}+A_{j-1} \hat{N}_{j-1}+\ldots\right.\right. \\
\left.\left.+A_{j-n+1} \hat{N}_{j-n+1}\right)\right] \exp \left(\frac{h}{2} \hat{D}\right) U\left(\xi_{j}, \tau\right)
\end{array}
$$


where the estimated $\overline{U\left(\xi_{j+1}, \tau\right)}$ is used in calculating the new $\overline{\hat{N}}_{j+1}$ according to Eq. (3.3) and the correction step is given by [71]:

$$
\begin{array}{r}
U\left(\xi_{j+1}, \tau\right)=\exp \left(\frac{h}{2} \hat{D}\right) \exp \left[h \left(C_{j+1} \overline{\hat{N}}_{j+1}+C_{j} \hat{N}_{j}+\ldots\right.\right. \\
\left.\left.+C_{j-n+1} \hat{N}_{j-n+1}\right)\right] \exp \left(\frac{h}{2} \hat{D}\right) U\left(\xi_{j}, \tau\right)
\end{array}
$$

where the coefficients $\mathrm{A}$ and $\mathrm{C}$ are dependent on the order number of predictorcorrector method $n$ as reported in [71].The PC-SSFM was found to be about 2.8 to 5.5 faster than the conventional S-SSFM [71]. For the purposes of this investigation the number of time points was chosen as a power of 2 to speed the FFT operations [72], specifically it was set to 2048, while the step size $h$ was decreased until a numerically convergent solution is obtained. In this work, the reported results and laser dynamics are confirmed in all three variations of the SSFM.

\subsubsection{LPFG Equation}

The simulation of the nonlinear coupled mode equations of the grating is straightforward as there is no dispersive term, thus Eqs. (2.71) and (2.72) are rewritten as:

$$
\begin{aligned}
& \frac{\partial U}{\partial \xi_{G}}=\hat{N}_{U} U \\
& \frac{\partial V}{\partial \xi_{G}}=\hat{N}_{V} V
\end{aligned}
$$

where $\hat{N}_{U}$ and $\hat{N}_{V}$ define the nonlinear grating operators for both core and cladding respectively, which are deduced from Eqs. (2.71) and (2.72) as:

$$
\begin{aligned}
& \hat{N}_{U}=i\left[\left(c_{11}|U|^{2}+2 c_{1 j}|V|^{2}\right)+d_{1 j} \frac{V}{U}-\frac{\Delta}{2}\right] \\
& \hat{N}_{V}=i\left[\left(c_{j j}|V|^{2}+2 c_{1 j}|U|^{2}\right)+d_{1 j} \frac{U}{V}+\frac{\Delta}{2}\right]
\end{aligned}
$$


The normalized SVEA of the electric field in the core and cladding propagating from $\xi_{G}$ to $\xi_{G}+h_{G}$ can be expressed in the following form:

$$
\begin{aligned}
& U\left(\xi_{G}+h_{G}, \tau\right)=e^{h_{G} \hat{N}_{U}} U(\xi, \tau) \\
& V\left(\xi_{G}+h_{G}, \tau\right)=e^{h_{G} \hat{N}_{V}} V(\xi, \tau)
\end{aligned}
$$

It is worth noting that the cladding fields are cleared as it reflects from the mirror shown in Fig. 2.1. The absence of the time derivative term in Eqs. (2.71) and (2.72), eliminates the need to perform FFT. Thus, the computational cost of solving the grating equations is less than that for solving the CGLE with its gain model.

\subsection{Object Oriented Programming}

Traditionally, procedural programming languages, such as Fortran, have been used in scientific and numerical computing [72]. Unfortunately, programs written in these languages are not easily extendable to meet new specifications brought about by either the development of programming concepts or the extension of the physical system or phenomena at hand $[74,75]$. In recent years, the use of object oriented programming (OOP) $[76,77]$ concepts in scientific computing has gained momentum $[34,78,79]$. For example, in computational electromagnetics, OOP concepts became vital to developing complex code used for hybrid time-domain numerical analysis [78]. Also, extendable and reusable OOP $\mathrm{C}++$ code utilizing inheritance, polymorphism and encapsulation has been developed for integrated optics modelling [79].

In procedural programming, data and functions are separate entities. Usually, data is predefined and function calls follow after. However, in OOP both are meaningfully integrated to form objects related to the problem domain [78]. As a consequence, OOP enables dynamic memory allocation at any stage of computing with a 
specific life time, thus, ensuring maximum generality and enhancing performance [74]. In the context of OOP an object is an instance of a class encapsulating both data and functions [76]. Attributes of a class are associated with a specific visibility such as private, public or protected [77]. Usually, data are defined as private to eliminate conflicting definitions [74], while most functions are defined as public forming a class interface [76,77]. The use of OOP for scientific computing enhances code development, maintenance, flexibility, portability and memory efficiency $[74,78]$. However, object interfaces which usually reside between the abstraction layer and the numerical subroutines hinders program performance as we shall demonstrate [80].

Initially, the laser modeled was programmed in MATLAB [33]. However, as high nonlinear effects require smaller step sizes, the interpreted program proved inefficient due to the increasingly high computational time. The choice was made to attempt programming in a non-interpreted OOP language such as $\mathrm{C}++$ [34].

The OOP design process involves developing objects representing concepts or entities from the problem domain [76]. In this work, a class for both complex numbers and vectors were developed. Here, a vector is defined as a dynamically allocated array of complex numbers. A simplified unified modeling language (UML) class diagram is shown in Fig. 3.2. The implementation of the SSFM methods lends itself to OOP encapsulation with separate nonlinear and dispersion operators in Eqs. (3.2) and (3.3), referred to as Nop and Dop respectively, allowing straightforward inclusion of the high order effects experienced at ultrashort pulse generation (see Chapter 5). The classes are relatively simple with the basic add, subtract, divide and multiply operators overloaded, which are deligated from vector to complex. The remaining sections of the program involve static methods which utilize both complex numbers and vectors. To assess the performance of the OOP model, Callgrind/KCachegrind a UNIX based software profiling and visualization tool [81], was used to find the functional blocks with the highest computational time. The results are shown in Fig. 3.3. 


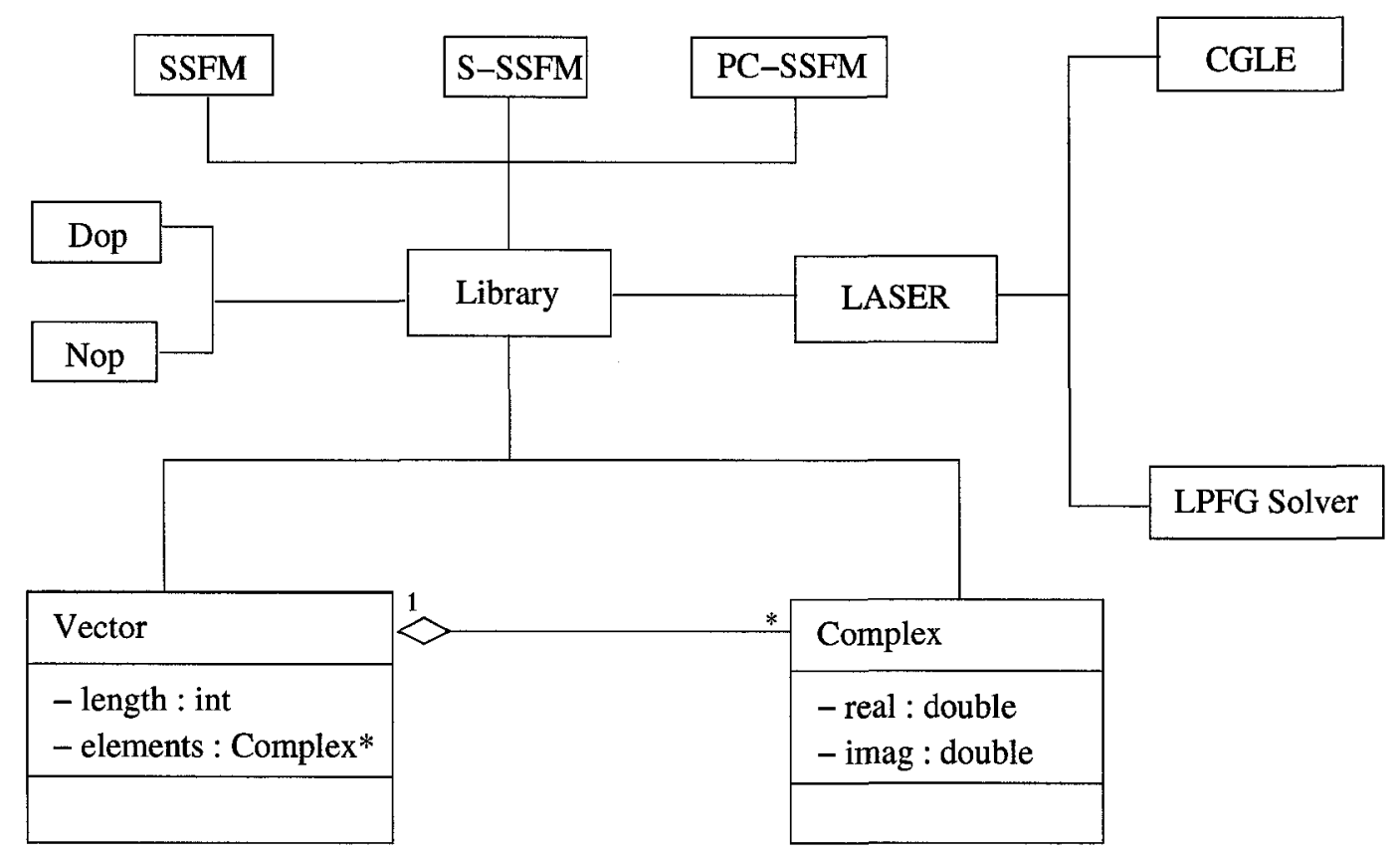

Figure 3.2: Simplified UML class diagram for the laser system implementation.

The simulation was started with low amplitude random light input and was allowed to circulate the laser cavity for 80 passes before producing pulses at a normalized saturated gain of $G_{0}=1.0$ with $\tau_{R}=0.0, \delta=0.0$ and $s=0.0$ [46]. The actual output of this simulation is reported in the next chapter, however, for now the focus is on implementation and performance. The profiling tool output shows that solving the CGLE requires more central processing unit (CPU) time than solving the grating equations. This is expected as the SSFM used in solving the CGLE requires a large number of FFT and inverse FFT (IFFT) operations. It is worth noting that, in Fig. 3.3 the vector constructor has a significant CPU usage. This is attributed to the overloaded operators used by the vector class [34]. OOP allows operators to be overloaded and declared with a keyword [74]. These operators implicitly call class copy constructors when pointer types are defined in class attributes $[78,82]$ as the case with our vector class. The total CPU time used was $334.47 \mathrm{~s}$ for 80 passes. 


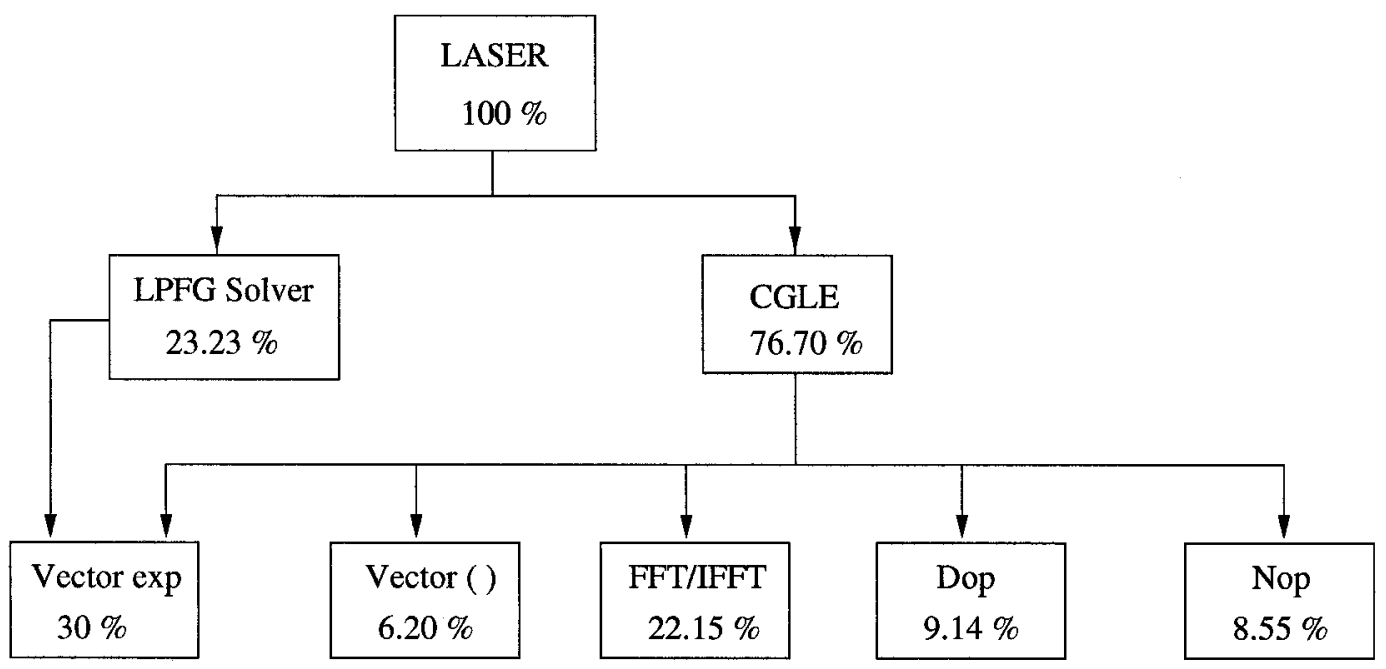

Figure 3.3: Program functional blocks with the highest computational time. The Callgrind/KCachegrind output.

In Fig. 3.2, the UML class diagram shows that class complex has both real and imaginary attributes set in private visibility. This design decision is common in OOP as data is hidden from class users as oppose to functionality, which form the class public interface [78]. As a consequence, to access or change these private attributes, for example real and imaginary, require separate functions known as accessors and mutators [74]. These are designed to ensure that instances of private variables are never used directly; instead, access is only done through these functions [77]. Although this ensures data security from invalid usage, it adds overhead to the computational time as these functions are called everytime access to complex numbers is required. A tradeoff exists between the level of data security and program efficiency. The same previous simulation was performed with both real and imaginary attributes being set to public visibility, hence eliminating the need for accessors and mutators. The total CPU time used in this new experiment was $296.09 \mathrm{~s}$, thus providing a 11.4 $\%$ efficiency improvement. These findings confirm with [80], where object interfaces which usually reside between abstraction layers and numerical subroutines hinder 
program performance. Thus, decreasing the level of encapsulation, reduces the computational time. This problem is experienced in all OOP languages used in numerical computations. Among all OOP languages, $\mathrm{C}++$ has attractive features for scientific computing [34]. Through optimized compilers C++ can be as efficient as its Fortran counterparts [83], however, new extentions to Fortran such as Fortran 95+ have included OOP features [84]. In essence, OOP concepts can be used efficiently to implement extendable and reusable $\mathrm{C}++$ code for scientific computing, permitting the rapid development and debugging of well-designed and compact code. However, an overhead is added from abstraction layers between object interfaces and numerical subroutines. A tradeoff exists between the level of data security and efficiency.

It is worth noting that inheritance, one of OOP main features, has not been utilized in our implementation. Inheritance is a method by which new classes (objects) are created based on older classes (objects). The new object, known as the child object, inherits attributes and behavior of the existing parent object [82]. In the laser model at hand, Eq. (2.65) is an approximation of three connected fiber segments, with one being an EDFA [32]. A more accurate simulation would involve propagating the laser pulse through three different segments. In a possible OOP implementation of such a system, inheritance could be used by setting a fiber parent class modeling normal fiber and child class modeling an EDFA. The child class would thus extend the parents behavior through fiber gain, hence encouraging code reuse. 


\section{Chapter 4}

\section{Laser Dynamics}

Instabilities in mode-locked laser pulse generation hinder its functionality, especially for critical applications where accuracy is vital. Hence, stability analysis plays an increasingly important role in laser design and modeling. Instability in a nonlinear optical ring cavity was first shown by Ikeda [85]. Using iterative methods and linear stability analysis, Ikeda showed that light circulating in a bulk cavity ring exhibits period doubling and eventual chaotic output. The operation of mode-locked lasers, essentially, involves high energy pulses actively or passively circulating in a nonlinear cavity. Thus, the dynamics seen by Ikeda could also be expected in mode-locked lasers. Although ultrashort mode-locked lasers normally generate soliton-like pulses, which are inherently stable, they have been found to exhibit period doubling bifurcation route to chaos in both theory and experiment [21-23].

In this chapter, the wide range of nonlinear dynamics exhibited by the laser shown in Fig. 2.1 are numerically investigated through solving the fiber Eq.(2.65), with the gain model governed by Eq.(2.66) and the LPFG Eqs. (2.71) and (2.72) in a selfconsistant manner. First, passive mode-locking and self-starting is studied under both Gaussian and random light inputs. Second, the laser dynamics are presented through plotting the increasing normalized gain against the pulse energy, so obtaining bifurcation diagrams. Finally, each of the dynamics observed is investigated separately. 


\subsection{Passive Mode-Locking}

Passive mode-locking is an all optical process, which does not involve any active modulation and is able of producing ultrashort optical pulses. It utilizes an intensity dependent nonlinear device to perform pulse shaping. In this laser system the LPFG acts like a saturable absorber causing this pulse shaping effect. The pulse width and the associated spectral broadening are limited by either the gain bandwidth or the LPFG transmission bandwidth [32]. To demonstrated stable mode-locking, a low intensity Gaussian light pulse is launched to start-off the laser mode-locking mechanism. The parameters used in this numerical investigation are physically reasonable and follow [32], with the resonant coupling occurring between the core mode and the third cladding mode $\left(d_{14}=12, c_{11}=c_{44}=0.2, c_{14}=20\right.$ and $\left.\Delta=0\right)$. The normalized length of the fiber is set to $\xi=1$, while the normalized grating length is set to $\xi_{G}=0.1$. The gain parameters are $\tau_{\Omega}=0.1$ corresponding to a gain bandwidth of $\Omega=25 \mathrm{~nm}$ and $E_{\text {sat }}=1$. The fiber loss is assumed to be $0.2 \mathrm{~dB} / \mathrm{km}$ and the output coupler is $5 \%$. In the simulation reported in Fig. 4.2, at a normalized gain $G_{0}=1.2$, the cavity light mode-locks forming a stable pulse after 15 round trips. Using the normalization in Chapter 2, the mode-locked pulse has a FWHM of $68.4 \mathrm{fs}$ and a peak power of $0.67 \mathrm{~kW}$. Both pulse width and peak power can be supported in EDFA cavities [86,87]. In Fig. 4.1 (a), the mode-locked pulse intensity is shown after 50 cavity round trips, while in Fig. 4.1 (b), the energy of the mode-locked pulse is plotted against the cavity round trips. Further, Fig. 4.2 (a) and (b), show the intensity profile of the mode-locked pulse with the cavity passes for both core and cladding respectively. The cladding modes are completley lost after each pass, as they do not couple back into the laser cavity. The pulse energy increases linearly as in Fig. 4.1 (b), before a slight fluctuation followed by a stable energy value. Increasing the gain will result in larger energy fluctuations leading to a diverse set of dynamics. 


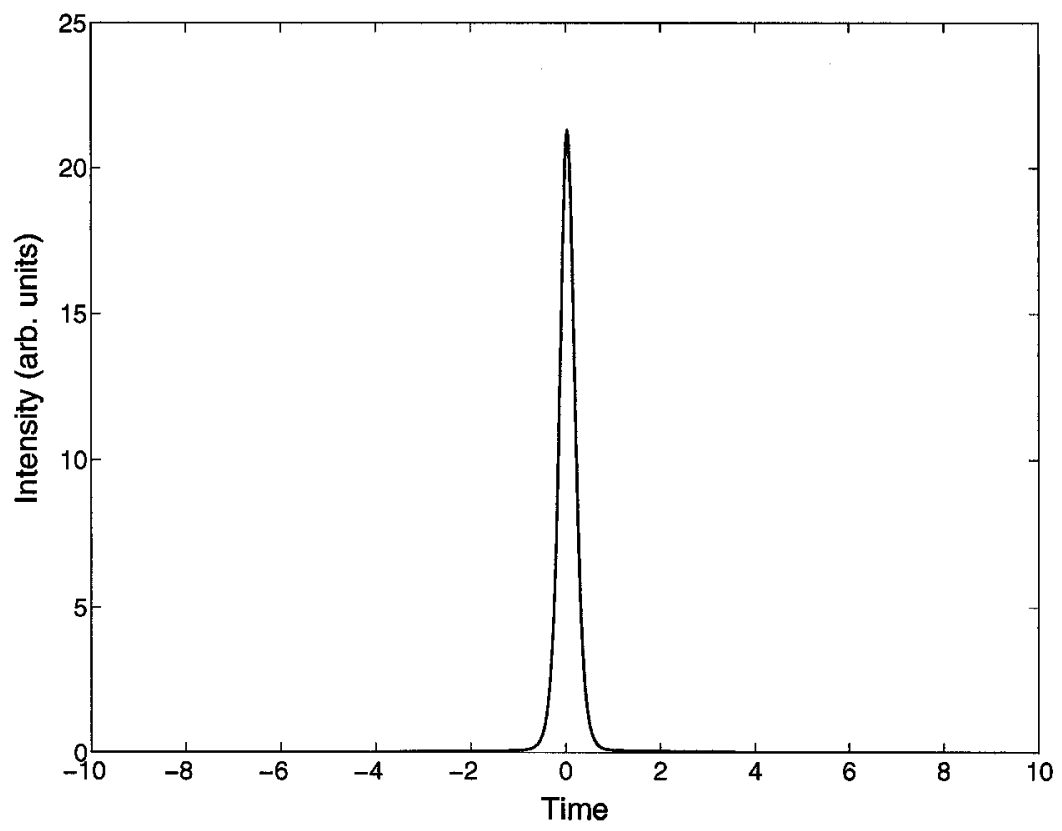

(a)

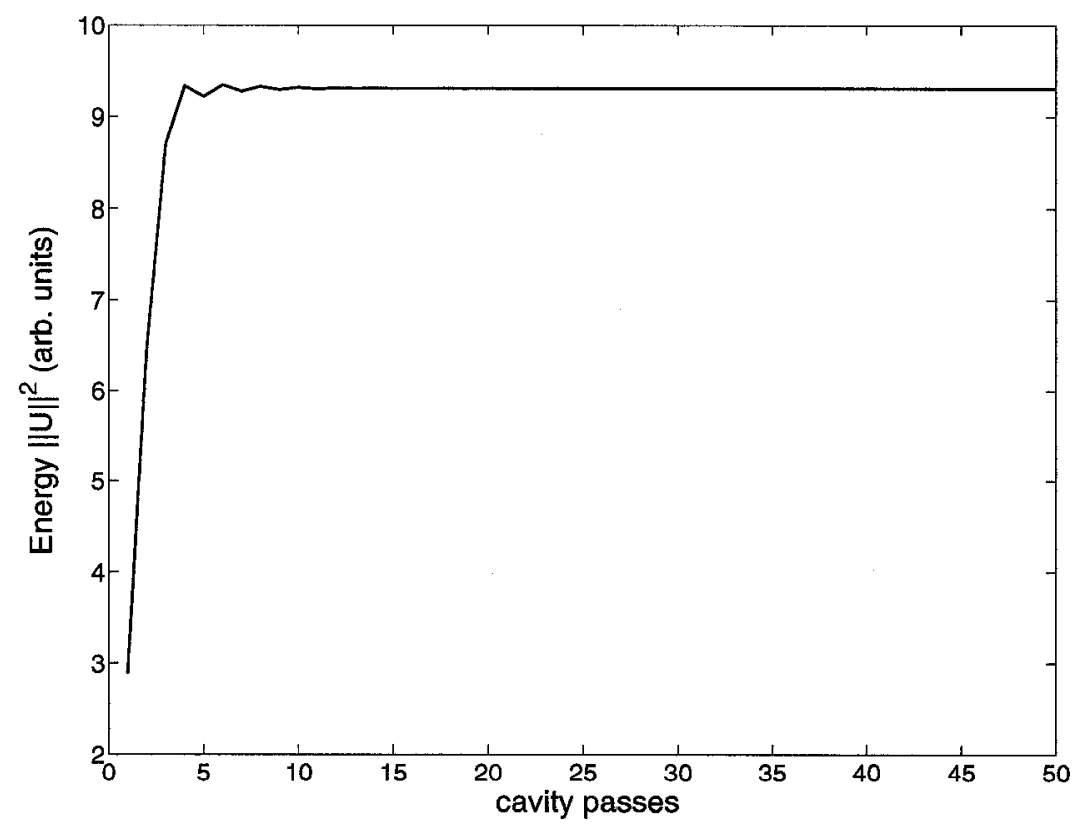

(b)

Figure 4.1: Stable mode-locked pulse dynamics for a low intensity Gaussian input pulse at $G_{0}=1.2$ (a) intensity of mode-locked pulse after 50 round trips. (b) energy of the mode-locked Gaussian input pulse as a function of cavity round trips. 


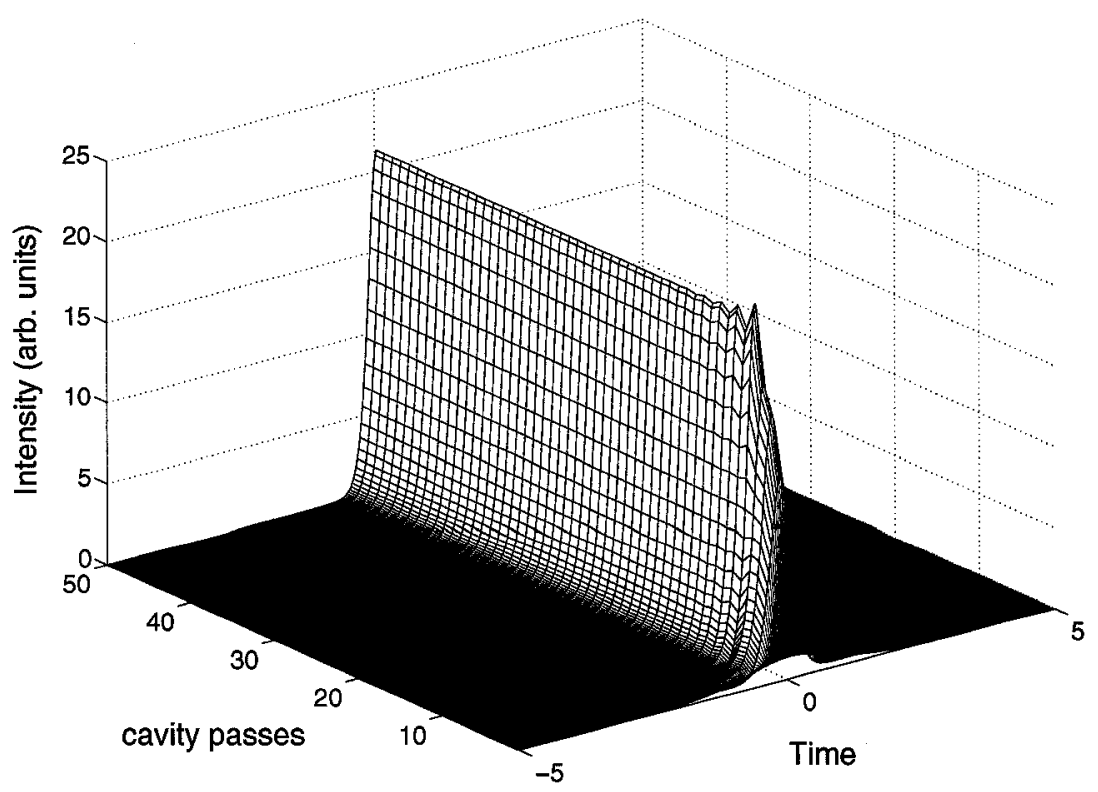

(a)

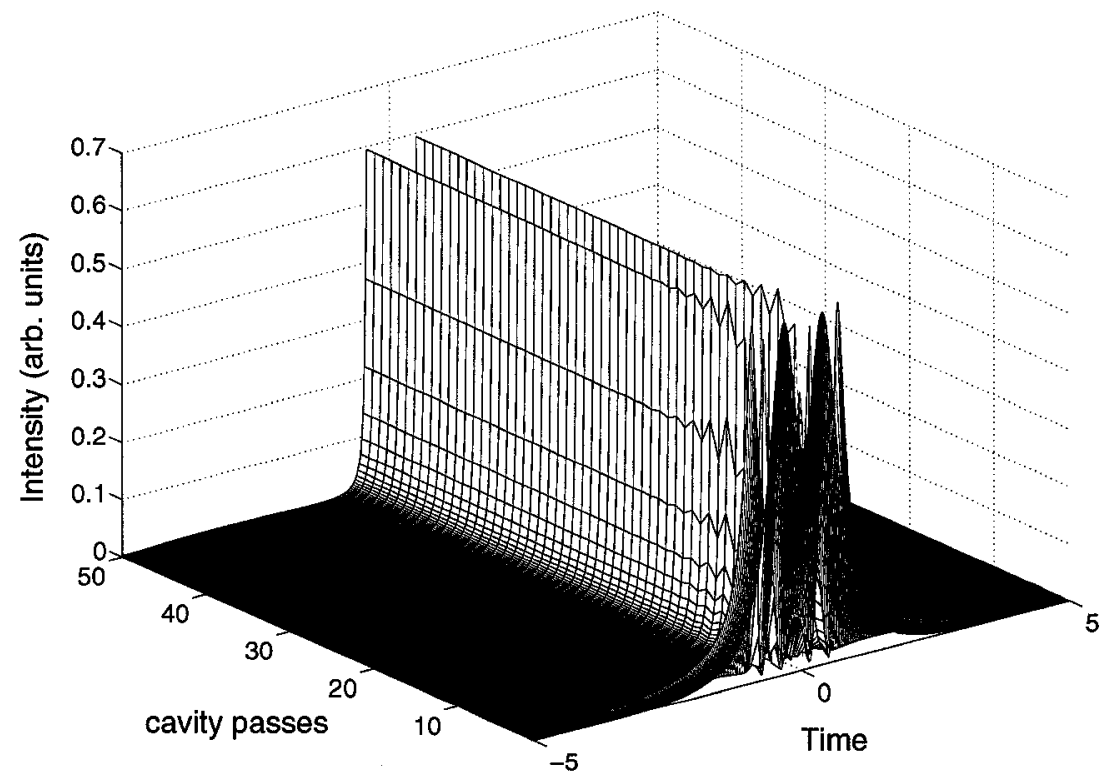

(b)

Figure 4.2: Intensity profile of stable mode-locked pulse as a function of cavity round trips for a low intensity Gaussian input pulse at $G_{0}=1.2$ (a) core profile. (b) cladding profile as an output of the LPFG. 
The previous simulation involved an artificial stimulation in the laser cavity manifested in the Gaussian input pulse. However, in practice, the possibilities of selfstarting is important. Thus, the next section explores the possibility of soliton selfstarting in the laser cavity.

\subsection{Autosoliton Generation}

In the previous section, the simulation involved a seeded Gaussian light pulse to start-off the mode-locking mechanism in the fiber laser cavity. However, self-starting soliton operation of mode-locked lasers has been reported $[88,89]$. To investigate the possibility of self-starting a low amplitude random light input was launched initiating a soliton at $G_{0}=1.2$. These types of solitons are usually referred to as autosolitons [19], which are unique solitary pulses forming in the laser cavity regardless of the initial input pulse width or peak power [20]. Autosolitons are soliton-like pulses supported by the CGLE, as oppose to the conventional solitons supported by the NLSE [90]. Hence, the EDFA can support autosolitons with width and peak power controlled by the amplifier gain and bandwidth [42]. In Fig. 4.3 (a), the mode-locked pulse intensity is shown after 200 cavity round trips. A standard sech $^{2}$ soliton pulse with the same peak value is also plotted. It is clear that the formed pulse is not an exact sech $^{2}$ soliton, but a soliton-like solution. Also, in Fig. 4.3 (b), the energy of the modelocked pulse is plotted against the cavity round trips. In Fig. 4.4 (a) and (b) the intensity profile and contour of the mode-locked pulse with the cavity passes is shown respectively. The laser cavity light requires 80 round trips to mode-lock producing stable pulses. From the plot in Fig. 4.3 (b), we observe two quantized energy levels. The first level assumes a value of 1.668 arbitrary units and occurs between the cavity round trips 15-37, while the other assumes a value of 9.3 arbitrary units and occurs at cavity round trips $>63$. This quantization can be attributed to the LPFG dynamics. 


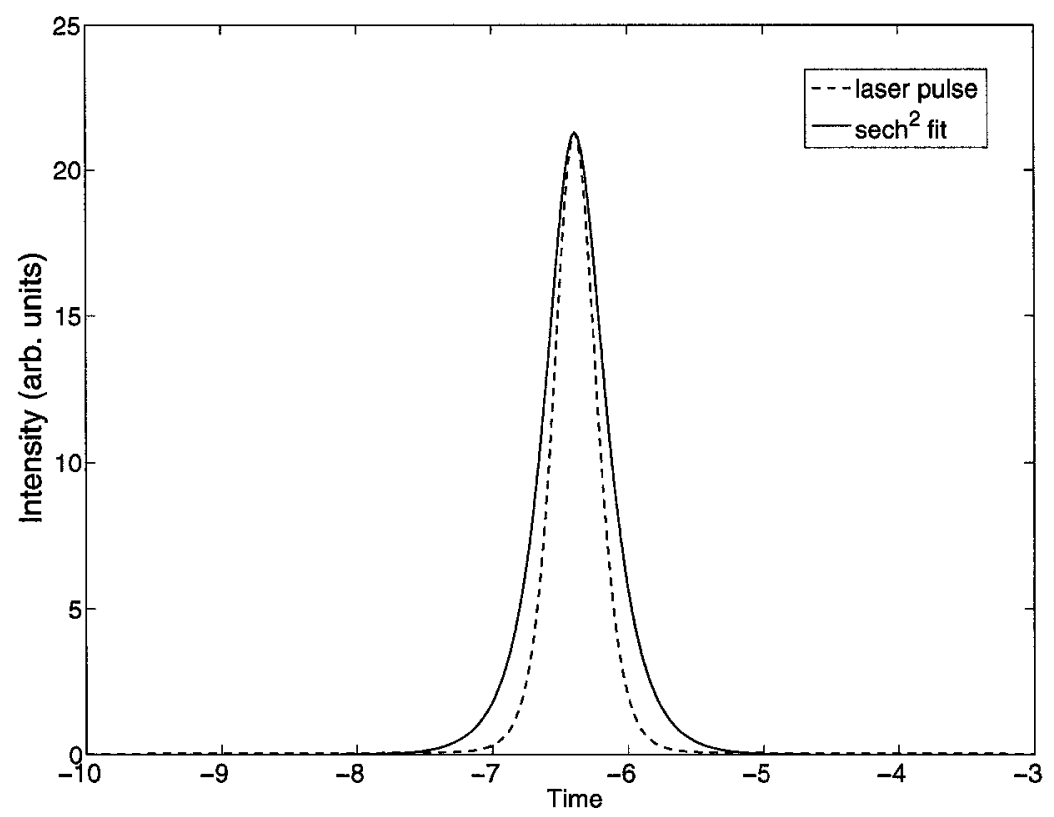

(a)

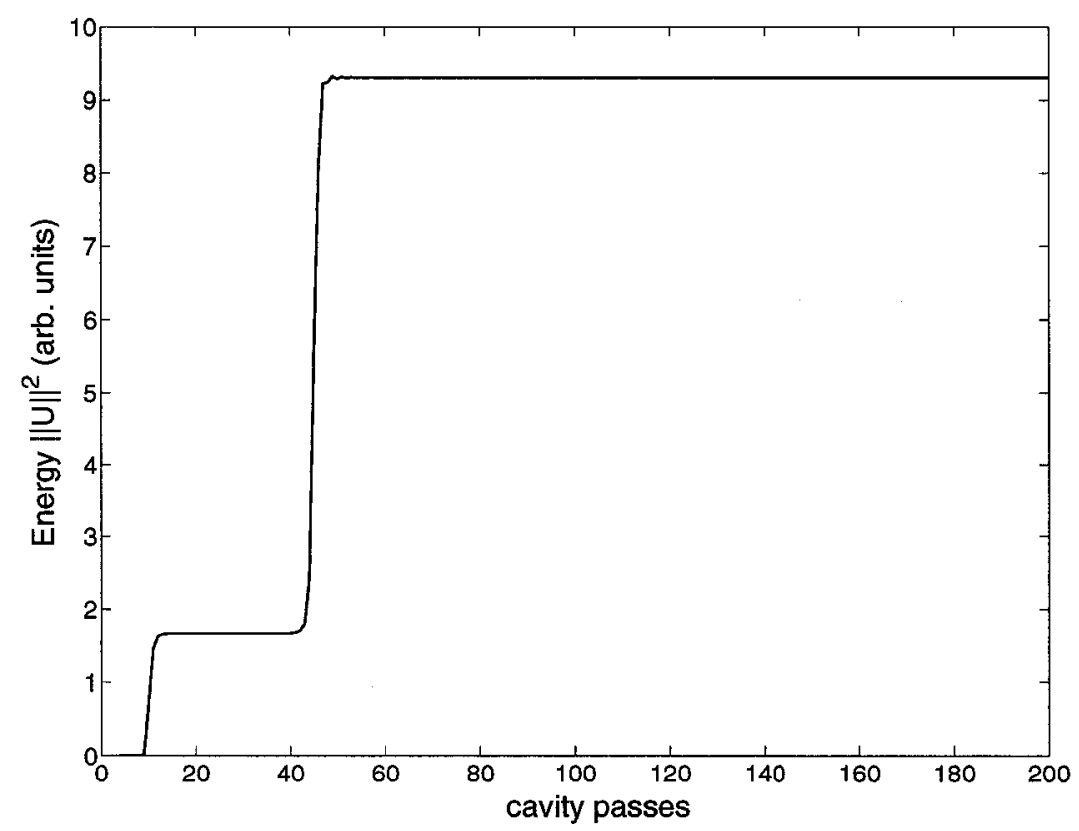

(b)

Figure 4.3: Stable mode-locked pulse dynamics for a low intensity random input pulse at $G_{0}=1.2$ (a) pulse intensity after 200 round trips with an exact soliton solution plotted. (b) energy of the pulse as a function of cavity round trips. 


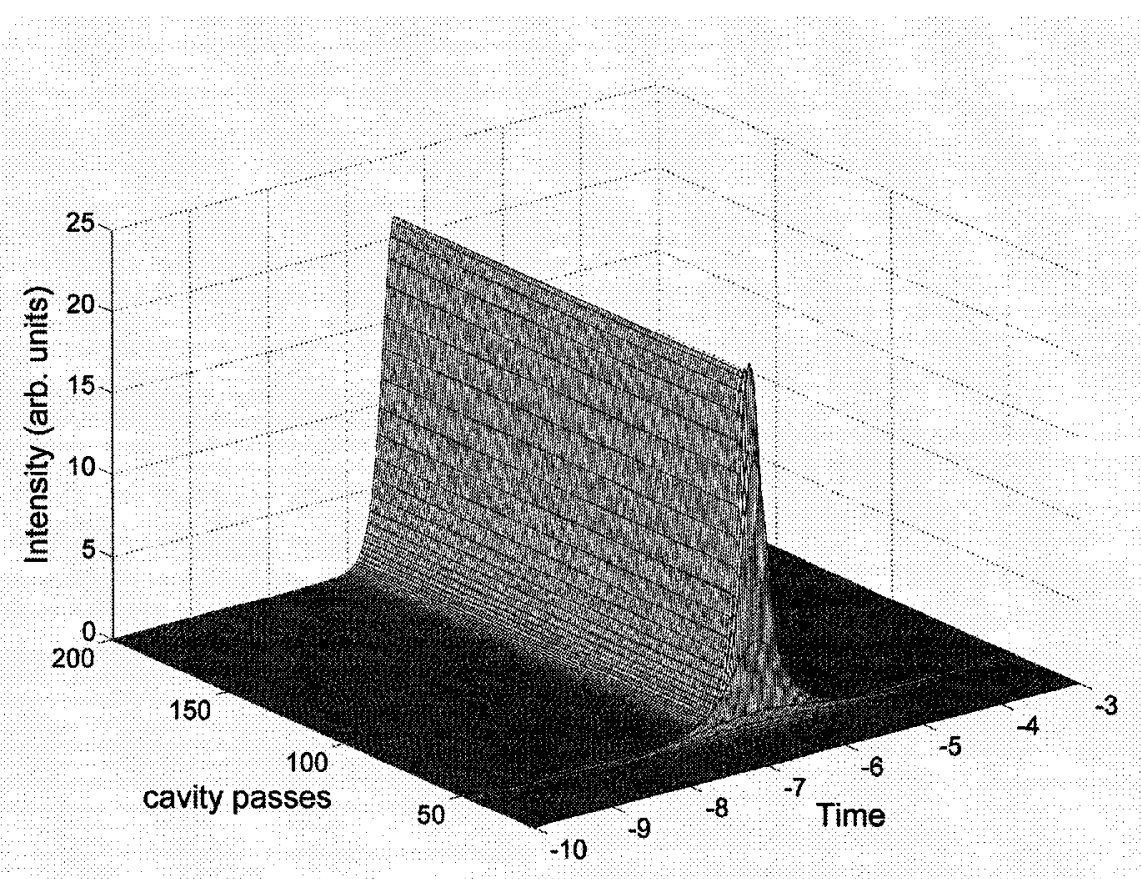

(a)

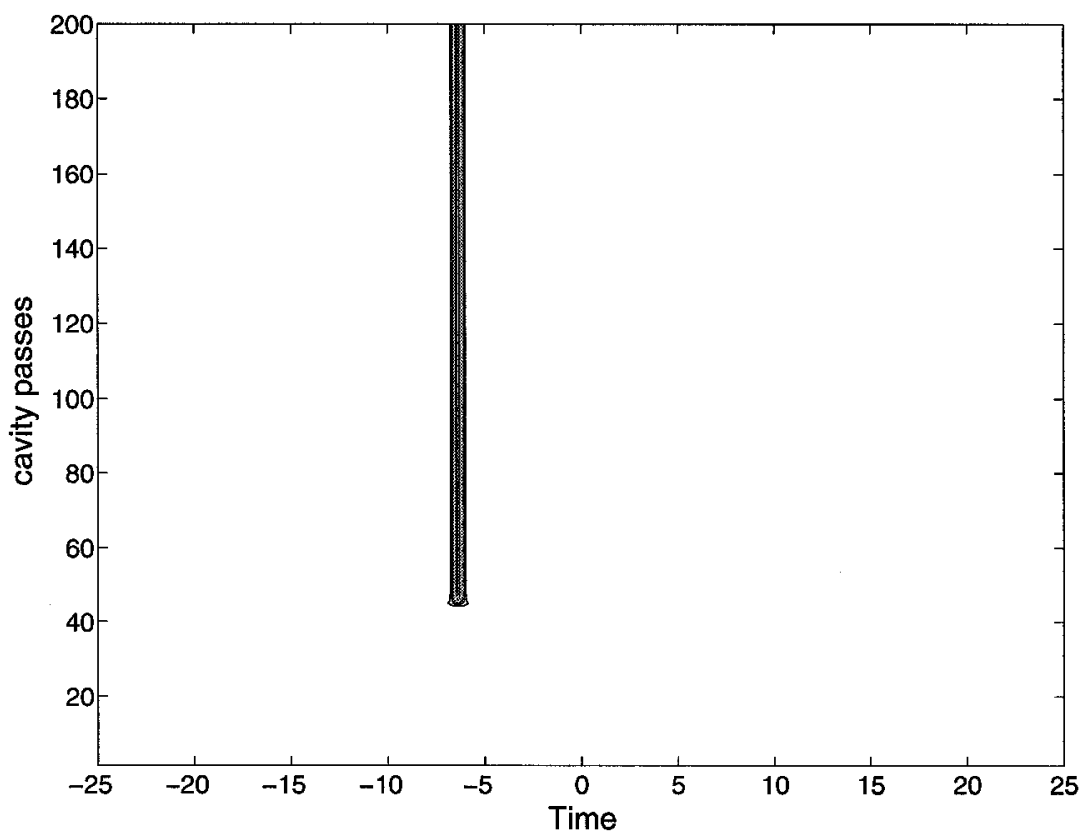

(b)

Figure 4.4: Self-starting autosoliton with a low intensity random light input pulse at $G_{0}=1.2$, with output intensity (a) profile. (b) contour. 
The LPFG filters any perturbation, resulting from cavity noise, to the mode-locked pulse in the first stable energy level, before the perturbation becomes too large to couple to the cladding. At this point, the EDFA amplifies the pulse resulting in an energy jump. Relating the energy quantization seen in Fig. 4.3 (b), to an autosoliton order may prove to be difficult, as the inverse scattering method is not applicable to the CGLE [39]. However, trial and error methods have been developed to find analytical expressions for an autosoliton order [91]. Unfortunately, for the model at hand, the CGLE is not the only element affecting the solitary-wave solution, and the nonlinear mode coupling equations of the LPFG need to be incorporated.

Initially the laser is trapped in a continuous wave $(\mathrm{cw})$ state $[88,91]$, before it mode-locks as a consequence of a perturbation. This perturbation can be a result of an external excitation, as the case with the Gaussian seeded input, or a result of the intrinsic noise in the cavity. In the second simulation the random light input is uniformly distributed over the time window, allowing this intrinsic noise perturbation. In fact, the work done in [88], formulates the self-staring time and shows that the process of self-starting of a passively mode-locking laser has a dynamical significance of a noise-activated crossing of an entropic barrier. However, the dynamics of the model were found to be very sensitive to the input power level and shape [88]. To examine the wide range of dynamics, two different noise input powers will be used, corresponding to low and high amplified spontaneous emission (ASE) in the EDFA [1]. The normalized average input power was chosen to be $10^{-11}$ arbitrary units and $10^{-3}$ arbitrary units corresponding to low and high ASE respectively. The choice of these values was determined from the normalizations presented in Chapter 2 and the experimental data for both forward and backward ASE in EDFAs [1]. The previous simulations involved only one cavity gain value at $G_{0}=1.2$. To investigate the wide range of laser dynamics, it is essential to explore higher gain values collectively. 


\subsection{Coexisting Attractors}

Soliton operation of passively mode-locked lasers has been conventionally modeled using the modified NLSE also known as the CGLE, which incorporates fiber nonlinearity, dispersion, loss and amplifier gain [2,9]. Laser models based on the CGLE were found to exhibit multisoliton formation and energy quantization [45]. These dynamics were reported in fiber lasers, such as the figure of eight laser [24], and the NPR laser [25]. In addition, the coexistence of multiple attractors, a general trend in dissipative driven nonlinear systems [26], has also been observed in modelocked lasers. For example, a $\mathrm{CO}_{2}$ laser with modulated losses have shown to exhibit multiple attractors for the same set of system parameters [27]. A generalized multistability analysis of erbium-doped fiber lasers with modulated losses based on rate equations also show coexisting attractors for photon density [28]. Experimental work on erbium-doped fiber laser with a sinusoidal pump modulation also shows the presence of coexisting attractors [29,30]. To investigate the coexisting attractors of the laser system in Fig. 2.1, all parameters were fixed, while the normalized saturated gain $G_{0}$ was increased from 1 to 4.5 for each level of ASE. Further, the laser dynamics are presented through plotting the pulse energy against the linearly increasing gain, so obtaining bifurcation diagrams. The following section is dedicated to this purpose.

\subsubsection{Bifurcation Diagrams}

Bifurcation diagrams are useful means of summarizing the complex behavior of a nonlinear system $[92,93]$. When plotting the pulse energy against the linearly increasing normalized saturated gain $G_{0}$ we obtain a bifurcation diagram for the laser model as shown in Fig. 4.5 and 4.6. At each gain value the laser model was simulated for 200 cavity passes to guarantee convergence, out of which the last 20 passes, namely from 180-200, were used in calculating the pulse energy. 


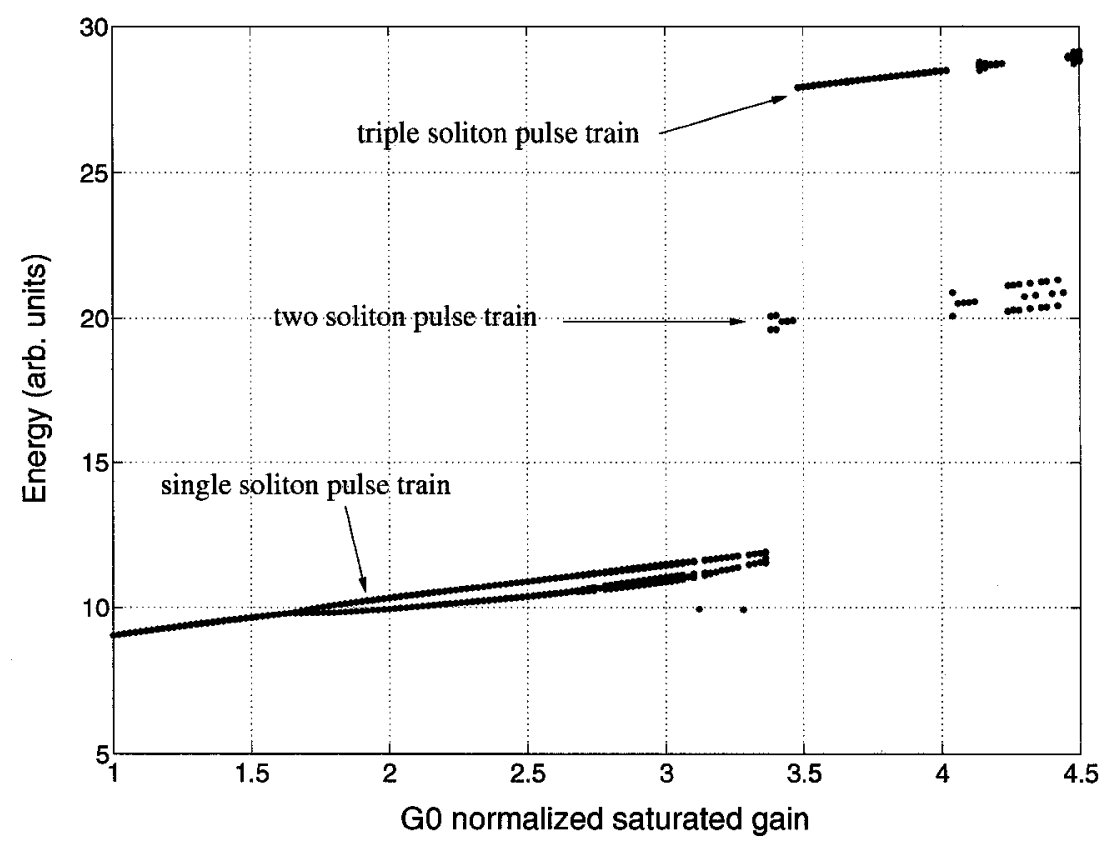

(a)

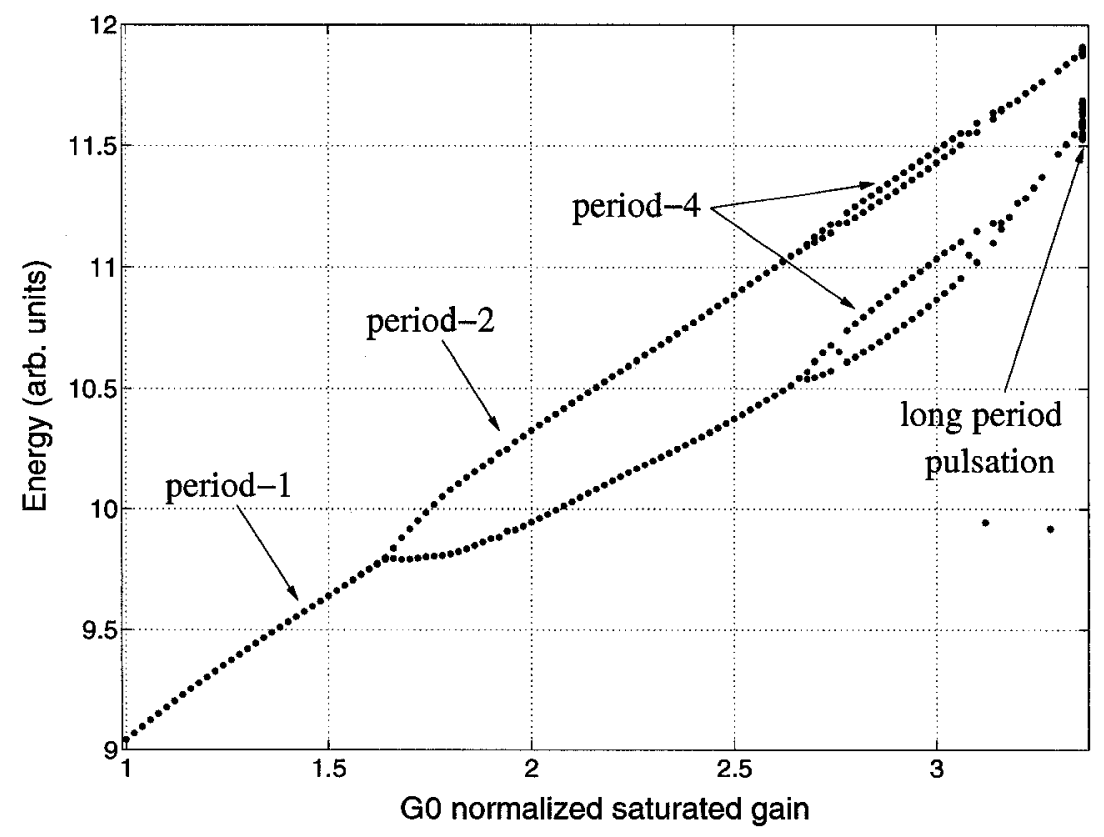

(b)

Figure 4.5: Bifurcation diagram of pulse energy $E$ plotted against the saturated gain for low ASE input (a) $G_{0}=1$ to 4.5 (b) expanded view for $G_{0}=1$ to 3.7 


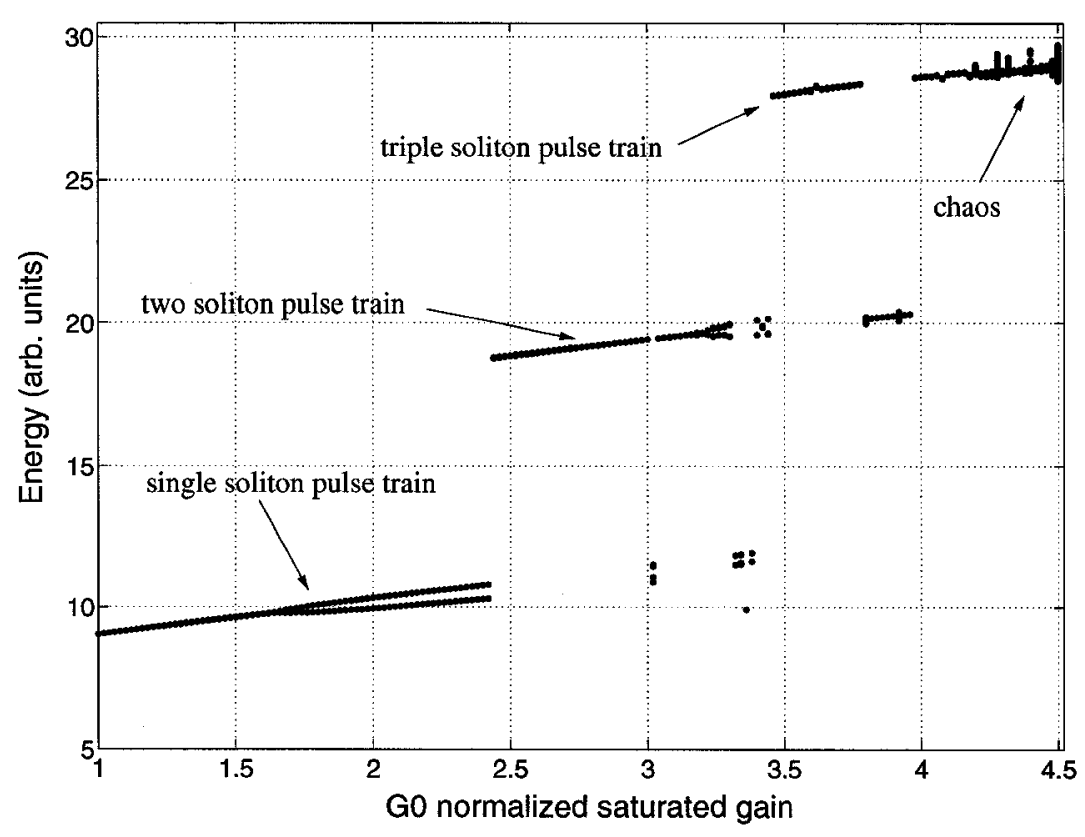

(a)

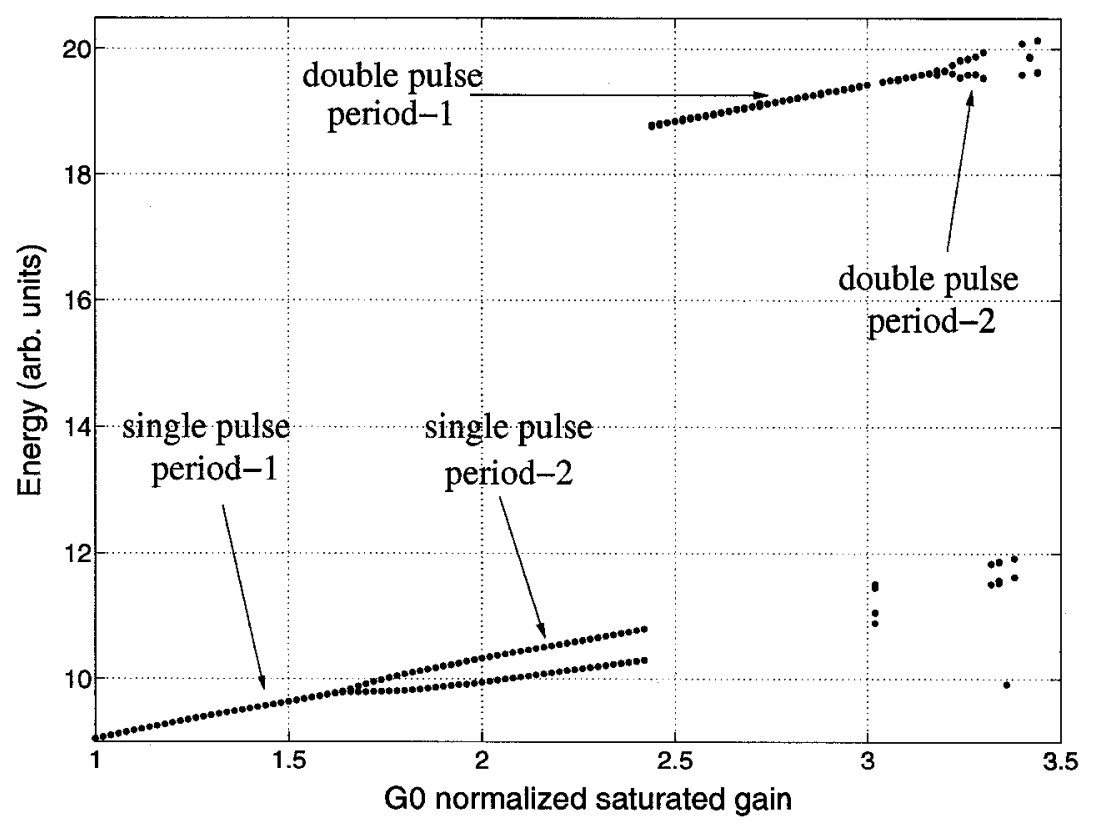

(b)

Figure 4.6: Bifurcation diagram of pulse energy $E$ plotted against the saturated gain for high ASE input (a) $G_{0}=1$ to 4.5 (b) expanded view for $G_{0}=1$ to 3.5 


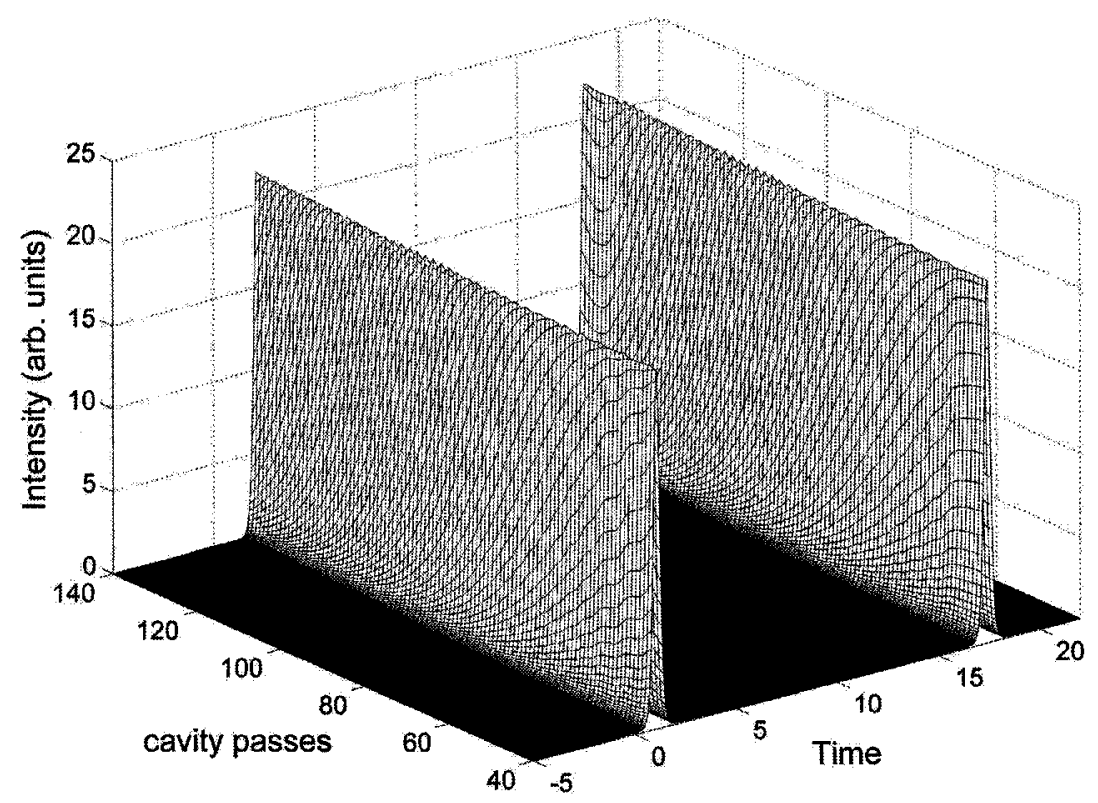

(a)

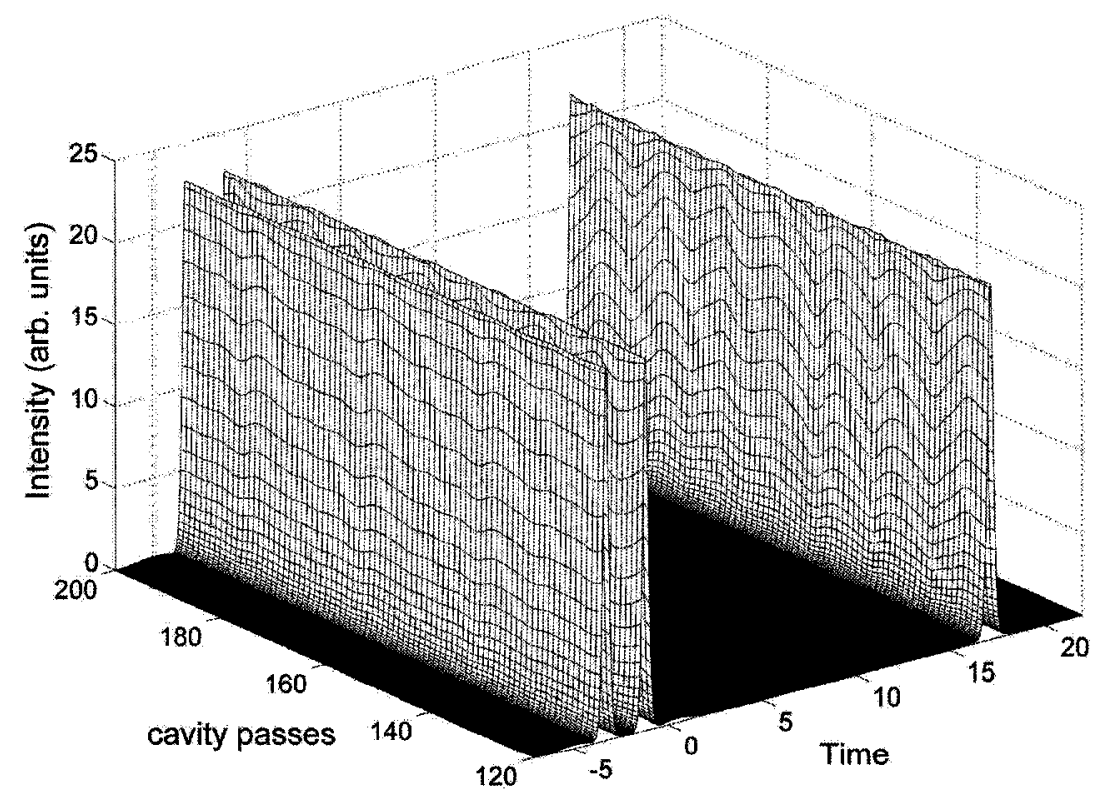

(b)

Figure 4.7: Soliton bound states (a) double soliton pulse train at $G_{0}=2.58$ with high ASE input. (b) triple soliton pulse train at $G_{0}=3.58$ with low ASE input. 
The bifurcation diagram shows possible coexisting attractors triggered by the formation of multisolitons in the laser cavity. As a consequence of the increasing pump power, the soliton pulse width reduces and peak power increases. However, the saturation energy of the erbium-doped model forms what is known as a "peak-powerlimiting" effect, which limits the maximum achievable soliton peak power $[25,45]$. The soliton pulse then divides into two trains forming bound states. In Fig. 4.5(a) and Fig. 4.6(a), three coexisting attractors are shown for low and high ASE inputs respectively. Each of the attractors relates to a distinct energy level, triggered by the formation of bound states. The three attractors correspond to single, two and three soliton pulse trains, with a normalized average energy of approximately 10, 20 and 30 arbitrary units respectively. The wide range of nonlinear dynamics from stability to bifurcation, long period pulsation and eventually chaos are present. However, it is worth noting that chaos is more likely to appear in attractors with higher average energy, as could be expected. In Fig. 4.5(b) and Fig. 4.6(b) an expanded view of the bifurcation diagrams are displayed corresponding to low and high ASE inputs respectively. An intensity propagation profile of the single soliton pulse train is reported in Fig. 4.4. The output intensity profile of two and three soliton pulse trains are shown in Fig. 4.7(a) and (b) respectively. The location of the soliton pulse trains is random, as it relates to the location of the random perturbations in the input noise. However, the closer the pulse trains are the more likely nonlinear energy transfer occurs between them, increasing the likelihood of instability and chaos. The following sections investigate the dynamics present in each attractor separately.

\subsubsection{Period Doubling}

The simulations in Fig. 4.5 and 4.6 indicate both single and bound soliton pulse trains exhibiting period doubling bifurcations. The propagation profiles of a single and bound soliton pulse train at $G_{0}=2.4$ and 3.4, respectively for low ASE are shown 
in Fig. 4.8. In Fig. 4.4, at the cavity gain of $G_{0}=1.2$, with low ASE input, the soliton pulse is stable with a uniform pulse energy and no bifurcation is observed. In other words, the laser outputs the same pulse shape and energy each round trip. However, as the pump power is increased to $G_{0}=2.4$, the peak intensity of the pulse train starts to fluctuate, alternating between two energy values as shown in Fig. 4.8(a). The two distinct energy values can also be seen in the bifurcation diagram of Fig. 4.5(b). In effect, period doubling is where the solitary pulse shape is repeated every other cavity round trip. Period four bifurcation can also be seen in Fig. 4.5(b). As the pump power is further increased to $G_{0}=3.4$, bound soliton states are formed, which also exhibit period doubling bifurcation. Fig. 4.8(b) shows the two soliton pulse trains simultaneously alternating between two distinct energy values.

\subsubsection{Long Period Pulsation}

In addition to period doubling bifurcation and chaos, the laser dynamics exhibit long period pulsation, which is observed in other lasers [21]. Fig. 4.9(a) and (b), shows the intensity profile and contour of long period pulsation at $G_{0}=3.36$, with low ASE input. Long period pulsation occurs when the soliton train has a period much greater than the cavity round trip time [21]. Whereas period doubling bifurcation is an example of short period pulsation, as the period is comparable to the cavity round trip time. It is worth noting that long period pulsation appears at the point prior to the excitation of the second coexisting attractor. In our example, the single soliton pulse train has a period of 18 round trip times. Observing the propagation profile shown in Fig. 4.9, it is possible to identify a slowly varying energy envelope with a period of 18 round trips. Long period pulsations have also been observed numerically and experimentally in passive mode-locked lasers [21]. The following section investigates laser chaos, which occurs at attractors with high average energy. 


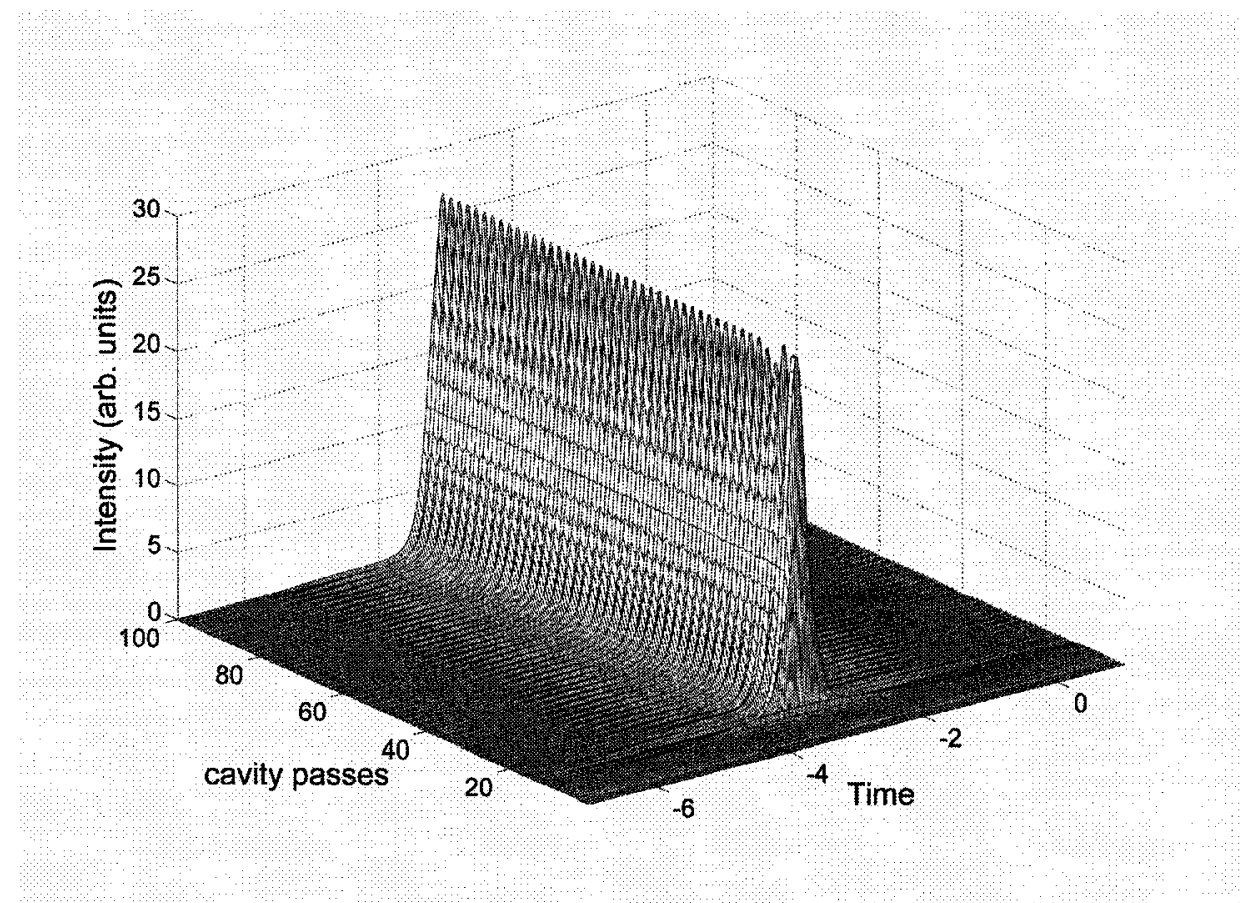

(a)

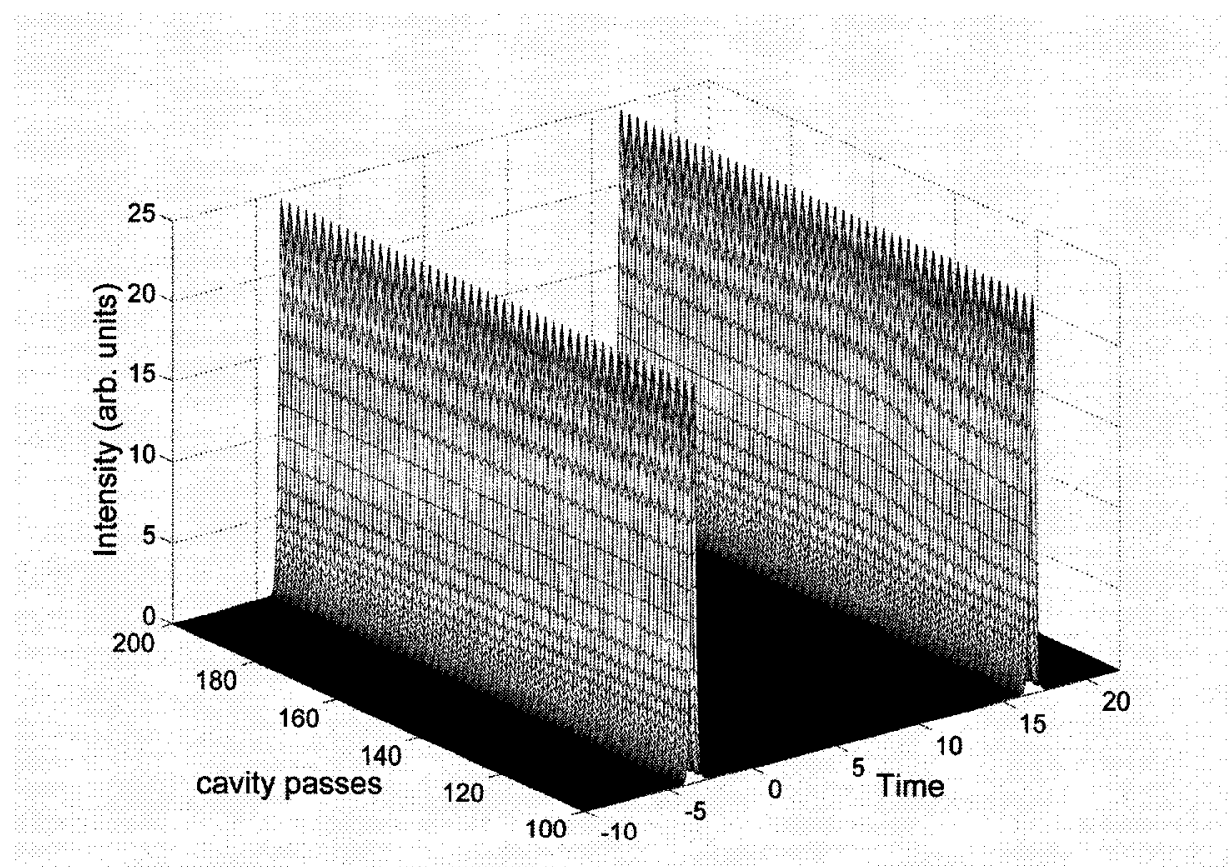

(b)

Figure 4.8: Period doubling of single and bound soliton pulse trains for low ASE input (a) period-2 single soliton pulsation at $G_{0}=2.4$ (b) period-2 double soliton pulsation at $G_{0}=3.4$ 


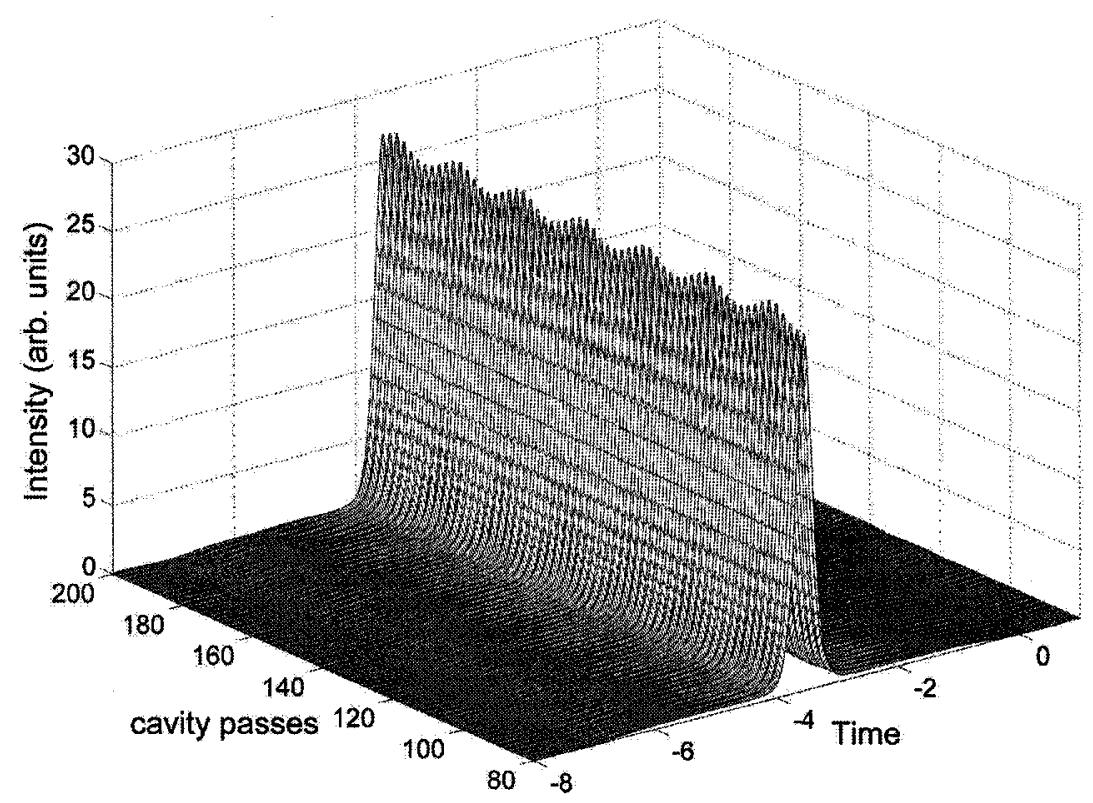

(a)

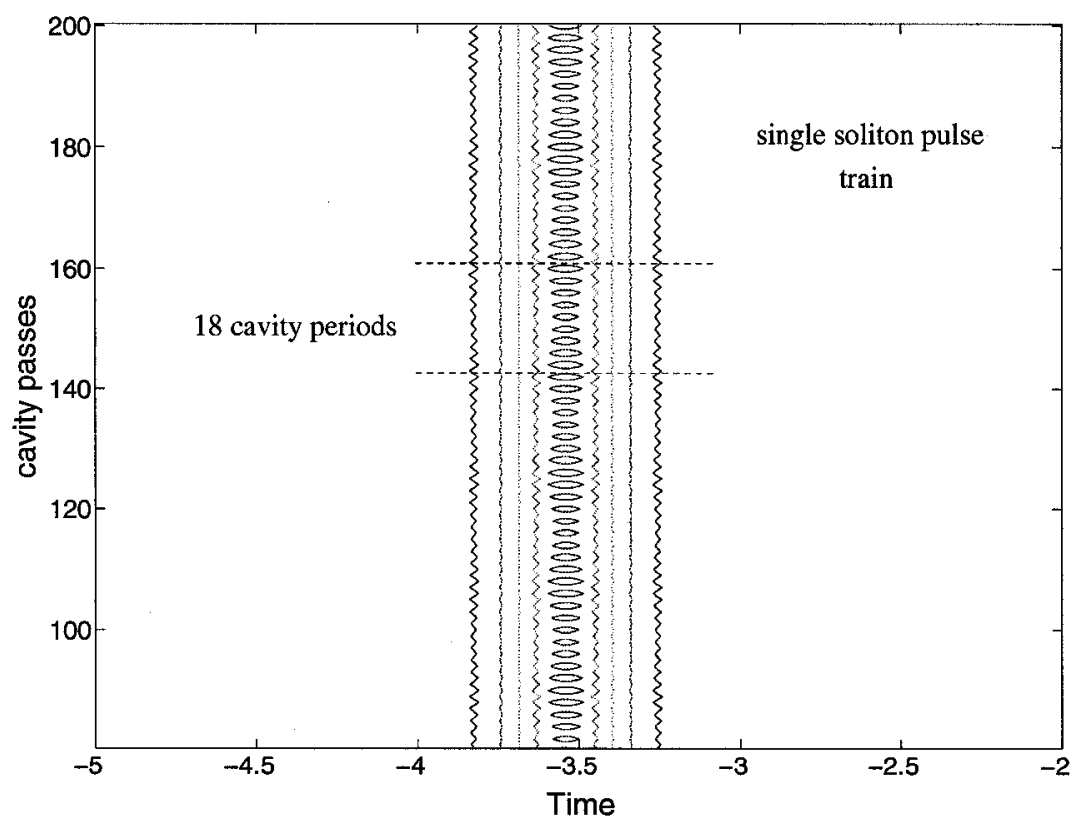

(b)

Figure 4.9: Long period pulsation at $G_{0}=3.36$ with low ASE input, with output intensity (a) profile. (b) contour. 


\subsubsection{Chaos Dynamics}

Solitons circulating the laser cavity undergo nonlinear mapping [21], which results in period doubling bifurcation and deterministic chaos [22]. The chaotic behavior experienced in these type of lasers are of great interest in theory and application $[27,29]$, and is also a source of laser instability at high pulse energies, which can be undesirable in some applications. For example, as the normalized saturated gain is further increased to $G_{0}=4.5$, with high ASE input, the triple soliton pulse train experiences deterministic chaos. In Fig. 4.10(a) and (b), the propagation profile and contour of this chaotic pulsation is shown respectively. This can also be seen in the bifurcation diagram of Fig. 4.6(a). The diagram in Fig. 4.11, where the pulse energy is plotted against the cavity round trips, shows high irregularity and chaotic fluctuations of the pulse energy, which experiences nonperiodic bursts of instability.

Erbium doped fiber lasers exhibit self-pulsation, a phenomena attributed to physical phenomena such as ion-ion interaction in erbium clusters or destabilization of relaxation oscillations [94]. It was found that self-pulsation leads to optical chaos in the laser output, following a period-doubling or a quasi-periodic route [95]. Optical chaos has been observed in erbium doped fiber lasers mode-locked using the NPR technique [22]. Although laser chaos is a source of instabilities, it has shown promise in applications for secure communications [96]. Chaos can be defined as deterministic behavior in a stochastic system, and exhibits the property of synchronization [92,97]. Two chaotic systems can be synchronized in a master-slave configuration and would produce the same output. This is a consequence of the deterministic nature of chaos. In a possible encryption system an information signal would have a chaotic signal, from the master, added to it, given the appearance of randomness, before it is transmitted. The receiver (slave) side would involve an identical chaotic system that is synchronized through the transmission. The original data is then obtained through 


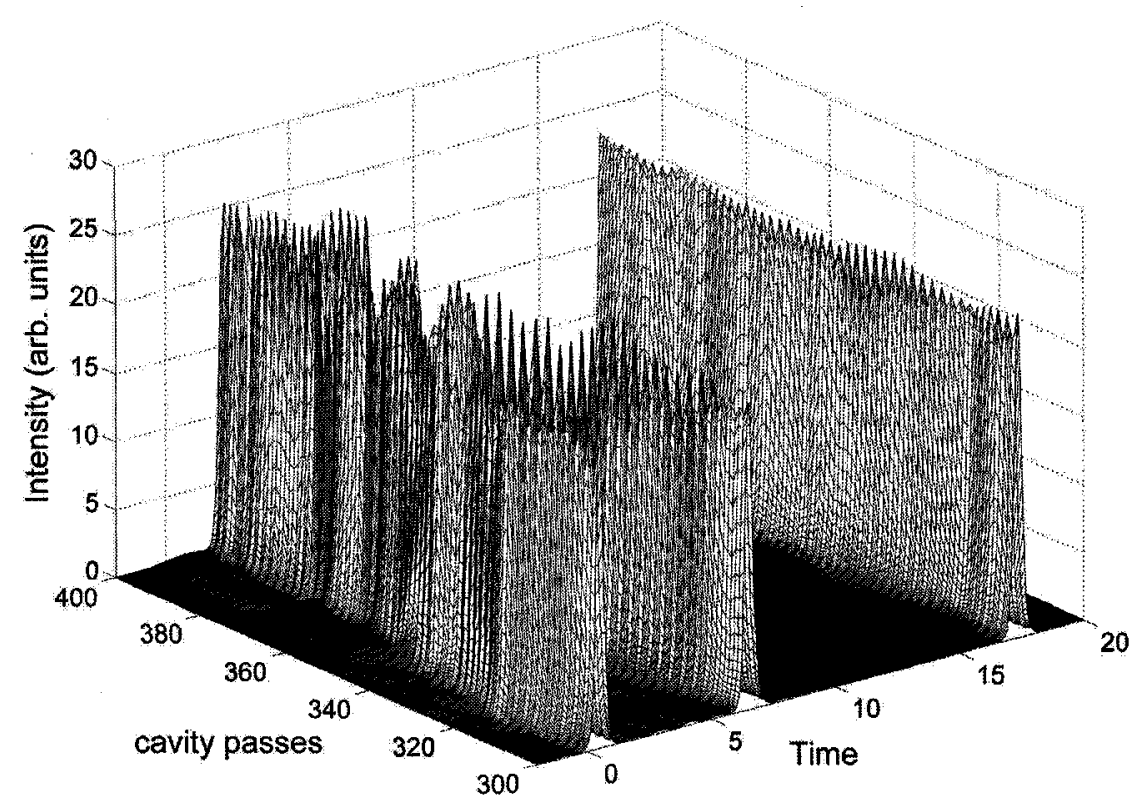

(a)

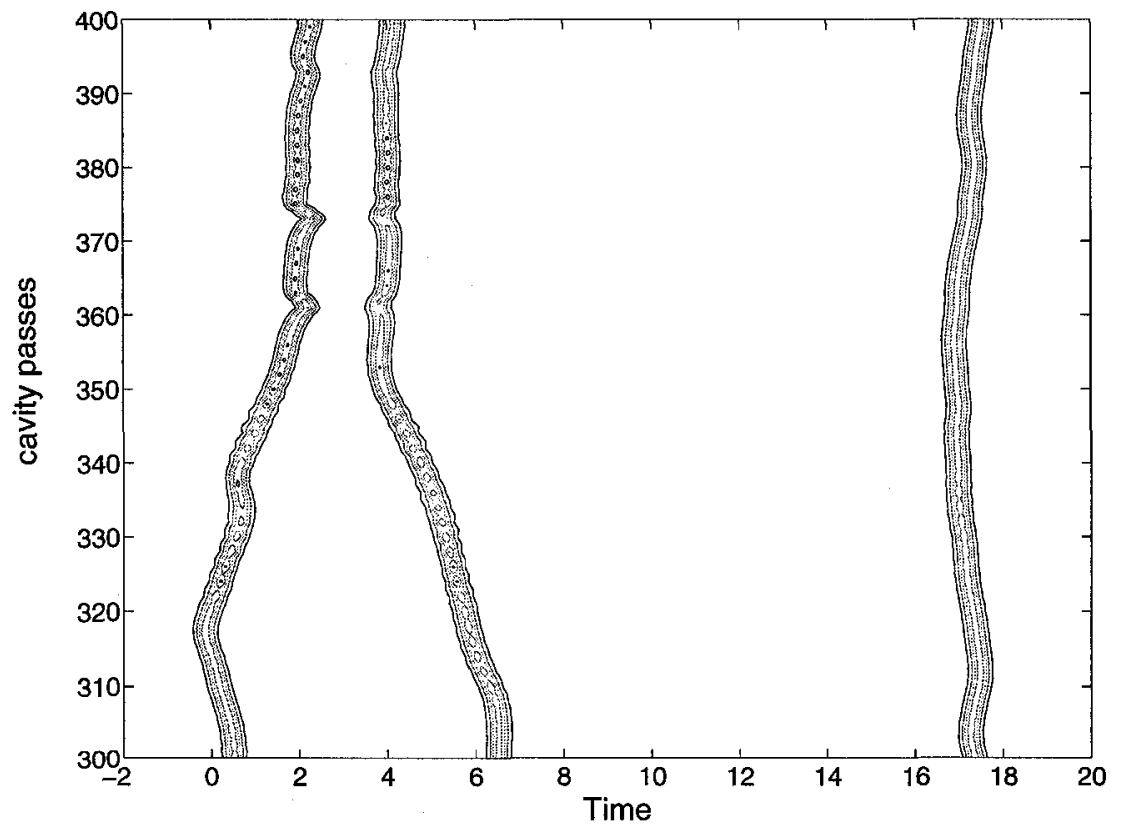

(b)

Figure 4.10: Chaotic pulsation for a triple soliton pulse train at $G_{0}=4.5$ with high ASE input (a) intensity profile. (b) intensity contour. 


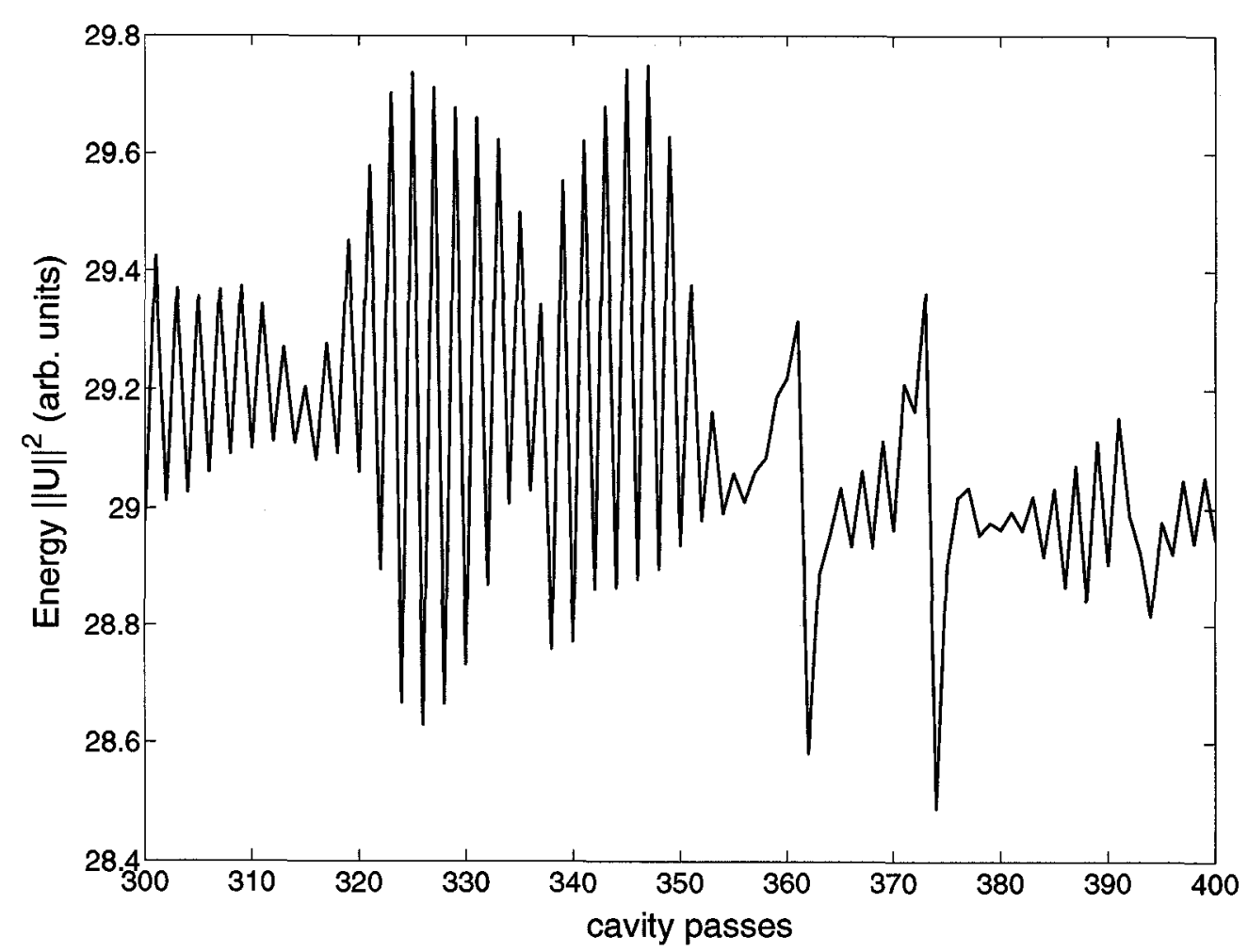

Figure 4.11: Pulse energy versus the cavity passes at $G_{0}=4.5$ with high ASE input.

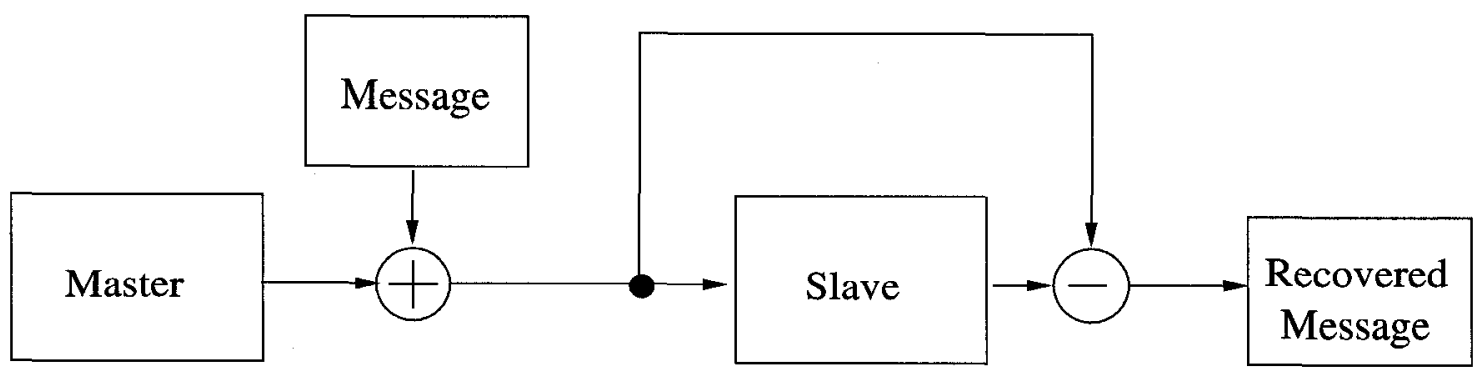

Figure 4.12: Chaotic secure communication block diagram. 
subtracting the transmitted signal form the regenerated chaos. An illustration of this process is shown in Fig. 4.12. Experimentally; there have been several successful attempts at employing chaotic fiber lasers for secure optical communications $[98,99]$. In one of these setups, a signal is masked with chaos through injecting it in the EDFA. The output signal is then transmitted through a $35 \mathrm{~km}$ fiber link, before it is injected in an identical EDFA at the receiver end for synchronization. The bit rate for this secure transmission has been found to $250 \mathrm{Mb} / \mathrm{s}$ [98] and even beyond $1 \mathrm{~Gb} / \mathrm{s}$ [99].

\subsection{Dynamics of the LPFG}

To further investigate the coexisting attractors, we explore the dynamics of the LPFG, which performs pulse shaping. So far we have observed the pulse at a fixed point in the cavity, namely the output coupler. This is an example of what is known as pulse macroevolution [21]. However, in the following investigation we look at the pulse evolution within the cavity, particularly in the core and cladding of the LPFG prior to the point it enters the EDFA.

The plot in Figs. 4.13 (a), (b) and Fig. 4.14 (a) show the light energy in the core $\|U\|^{2}$ and the light energy in the cladding $\|V\|^{2}$ plotted against the laser passes for three different gain $G_{0}$ values, each corresponding to one of the three attractors for low ASE input, respectively. Initially, the energy in the core and cladding are negligible. As the laser passes increase, the pulse shaping mechanism induced by the LPFG performs intensity discrimination, allowing the low intensity portions of the pulse to be coupled to the cladding and attenuated, while the high intensity portion is transmitted efficiently in the core [32]. After mode-locking the core light energy stabilizes at a high value, while the cladding energy becomes sufficiently low, as less light is coupled out to the cladding. In Figs. 4.13 (a), (b) and Fig. 4.14 (a), observing $\|U\|^{2}$ we can distinctly see the average normalized energy of about 10, 20 


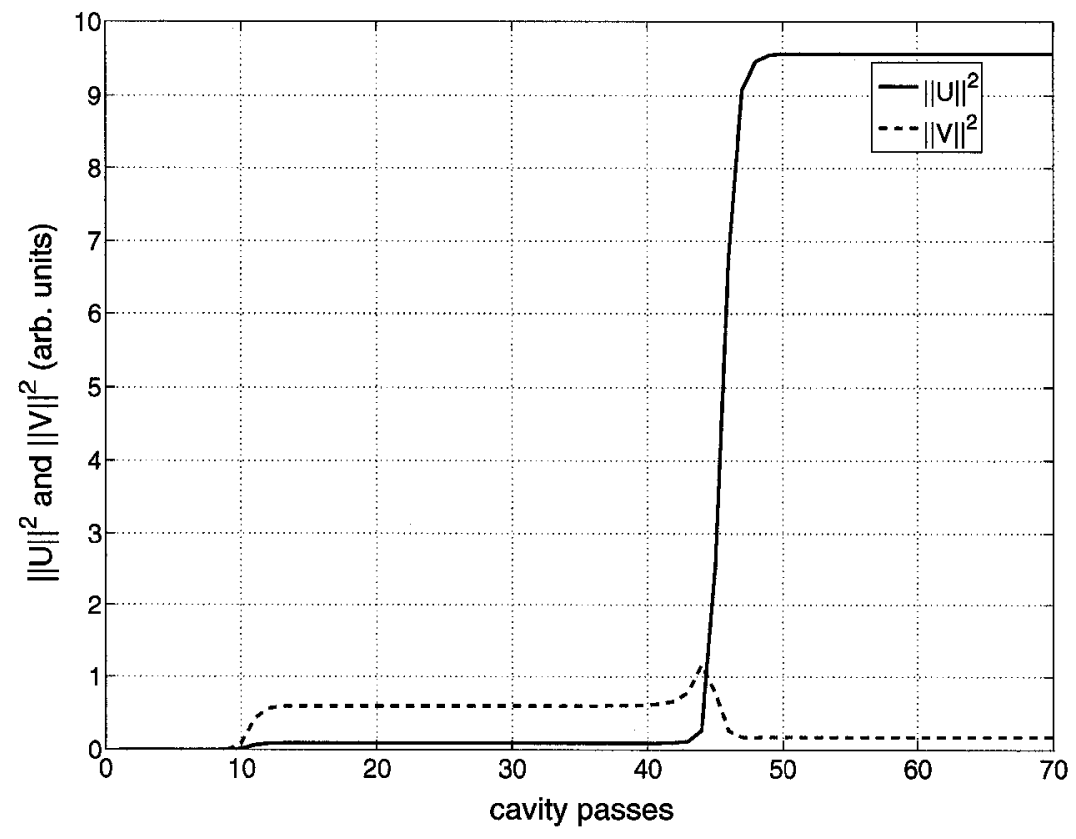

(a)

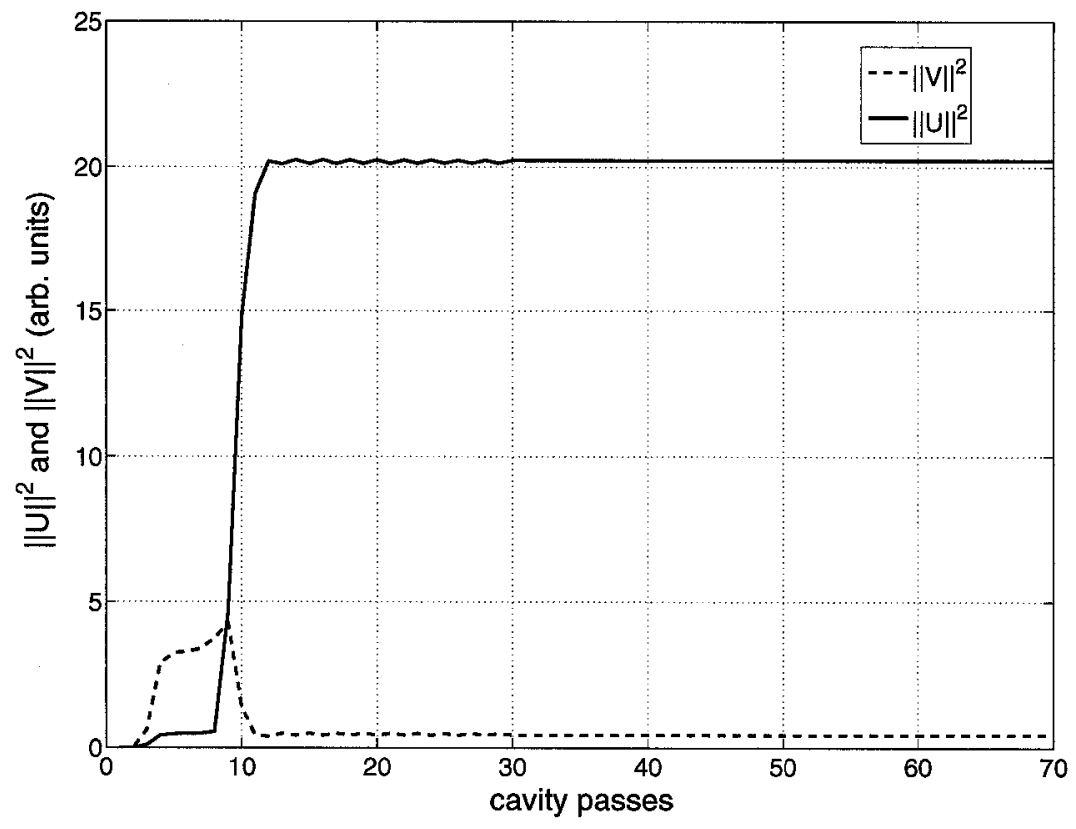

(b)

Figure 4.13: LPFG Dynamics with cladding light energy $\|V\|^{2}$ and core light energy $\|U\|^{2}$ in the LPFG plotted against laser cavity passes with low ASE input for (a) $G_{0}=$ 1.2 (b) $G_{0}=3.42$ 


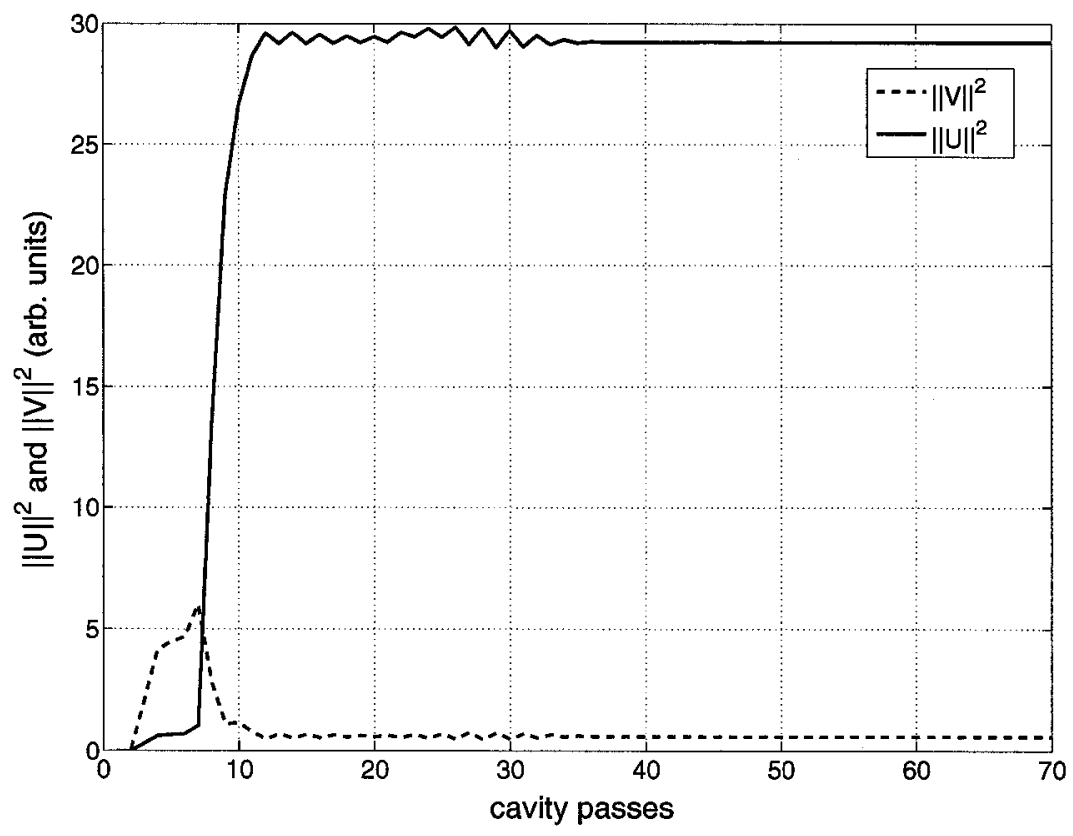

(a)

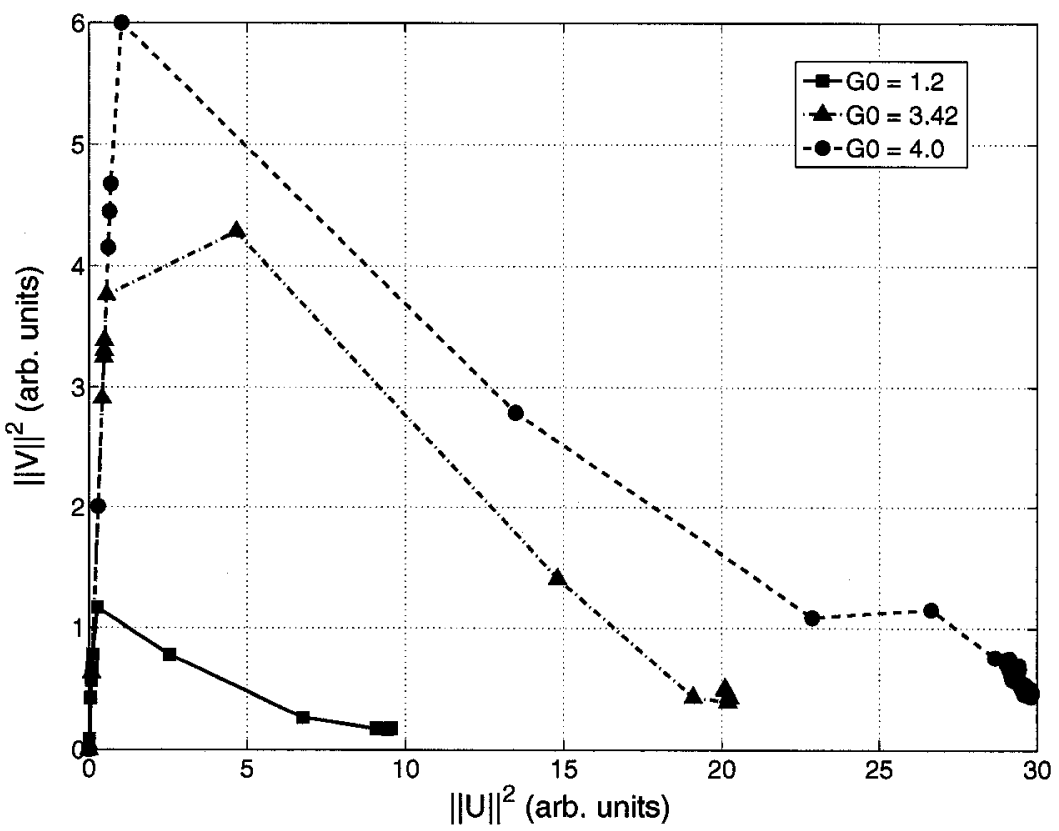

(b)

Figure 4.14: LPFG Dynamics: (a) cladding light energy $\|V\|^{2}$ and core light energy $\|U\|^{2}$ in the LPFG plotted against laser cavity passes with low ASE input for $G_{0}=$ 4.0 (b) $\|U\|^{2}$ plotted against $\|V\|^{2}$ for three gain values $G_{0}=1.2,3.42$ and 4.0 
and 30 arbitrary units corresponding to each coexisting attractor. As the gain $G_{0}$ increases the laser system requires less number of passes to mode-lock. It has been shown in [88], that the buildup time does not scale with the inverse of the cavity gain power, however, it is exponentially related. The authors show that the process of self-starting of a passively mode-locking laser has a dynamical significance of a noise-activated crossing of an entropic barrier. The plot in Fig. 4.14 (b) , shows $\|V\|^{2}$ plotted against $\|U\|^{2}$ for the same three different gain $G_{0}$ values. Initially, all core and cladding energy relationships are the same for all gain values, namely linear increase in the amount of light coupled into the cladding verses that which is coupled into the core. However, the nonlinear mode-coupling dynamics of LPFG force the system to mode-lock with a fixed amount of light coupled into the cladding each pass. For higher gain values, the linear segment of the $\|V\|^{2}$ versus $\|U\|^{2}$ relationship is longer, meaning that more light is coupled into the cladding prior to mode-locking. This is expected as more soliton pulse trains are formed. In Fig. 6 (d), we can also distinctly see the average normalized energy of about 10,20 and 30 arbitrary units, corresponding to each coexisting attractor. A detailed study of the theoretical and experimental aspects of the nonlinear propagation in the LPFG was performed in [35].

\subsection{Soliton Sidebands}

A characteristic feature of the instabilities of mode-locked lasers whose fundamental means of operation is based on soliton-like pulse shaping, is the emergence of one or more discrete spectral lines in the wings of the soliton spectrum. These spectral sidebands have been reported in figure of eight lasers, NPR technique lasers and semiconductor lasers [2]. The presence of these sidebands shifts energy to the pulse wings, which affect stable mode-locking operation. Based on the numerical model, we further investigate the soliton features in each of the period doubling stages. At a 


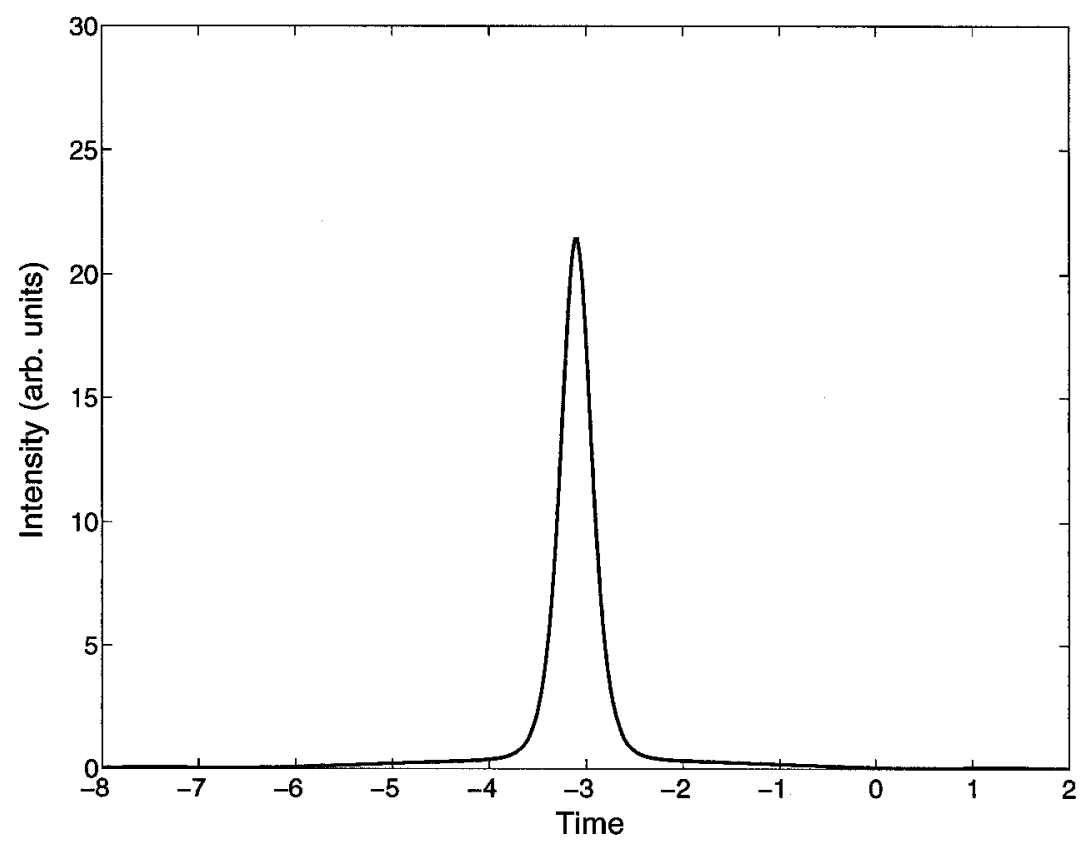

(a)

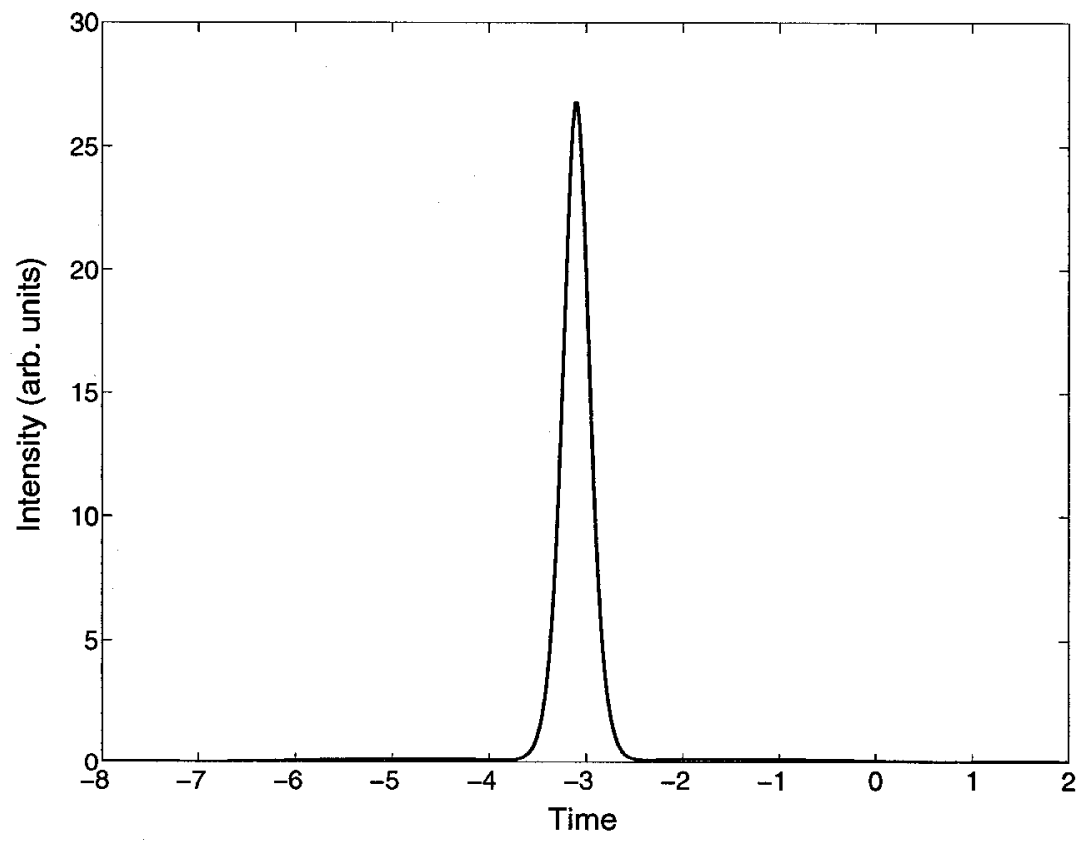

(b)

Figure 4.15: Period doubling temporal soliton pulse at $G_{0}=2.4$ with low ASE input (a) round-1. (b) round-2. 


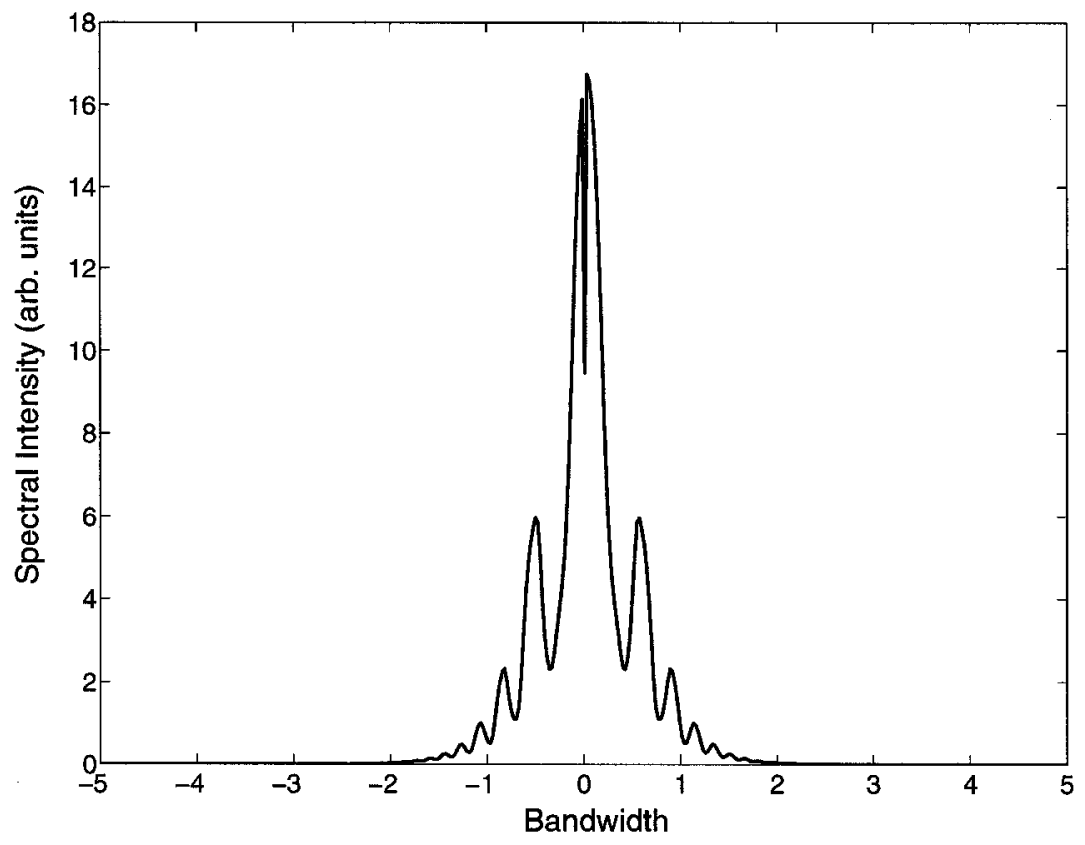

(a)

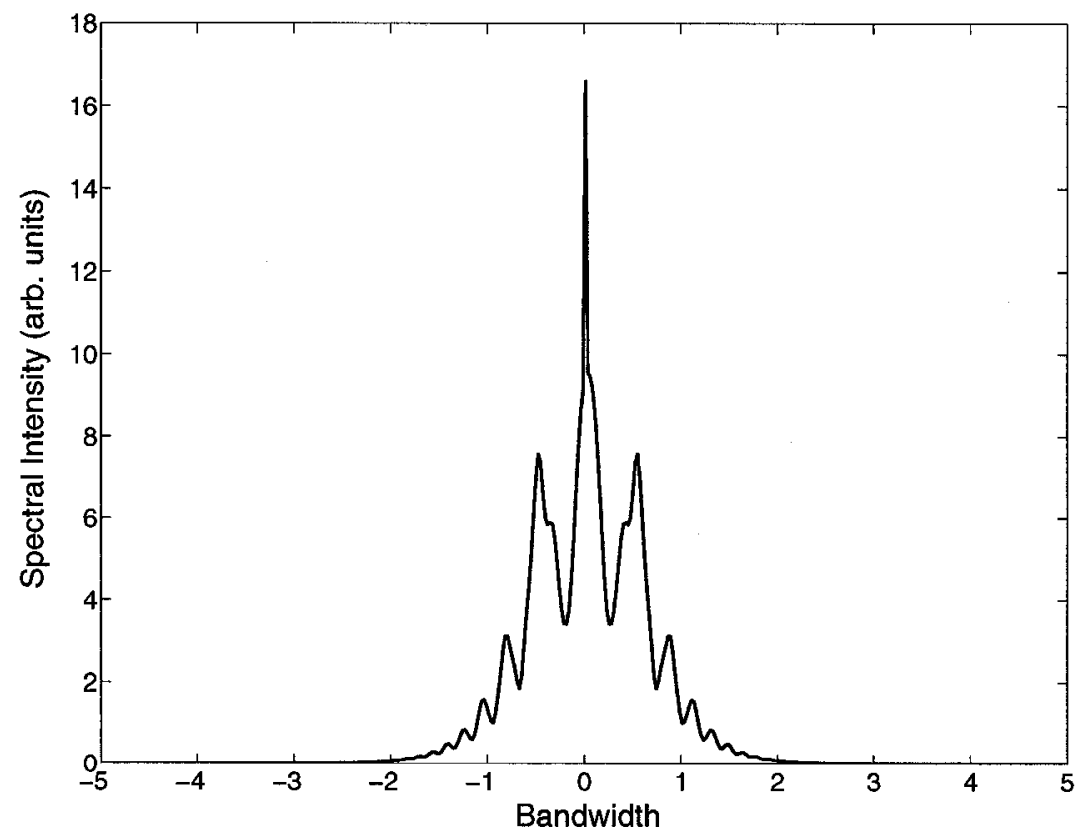

(b)

Figure 4.16: Period doubling spectral soliton pulse at $G_{0}=2.4$ with low ASE input (a) round-1. (b) round-2. 
normalized saturated gain $G_{0}=2.4$, with low ASE input, we plot both temporal and spectral soliton after each round trip as shown in Fig. 4.15 and Fig. 4.16, respectively.

The first round trip results in a soliton spectrum similar to that of a stable pulse train. The second round trip results in a different spectrum were extra soliton sidebands appear similar to those reported in other laser systems [22]. These sidebands can be attributed to spectral resonances associated with the periodic perturbations to the soliton caused by the cavity [2].The frequency and amplitude of these sidebands can be calculated using perturbation theory of solitons [42].

The work done by [2], shows the sidebands appearing to extend over the full extent of the optical energy even outside the fit for the conventional hyperbolic secant soliton shape. It has been shown, that due to the laser gain and output loss the parameters of the soliton formed in the cavity changes periodically $[21,22]$. As a result of this periodic perturbation, at each round trip a non-soliton portion is generated travelling as a dispersive wave [2]. The soliton and dispersive wave will propagate with different phase shifts resulting in a wavelength dependent phase accumulation process. As a result certain frequencies will induce constructive and destructive interferences forming soliton sidebands [2].

Further, the effect of third order dispersion on soliton sideband generation is significant [100]. Assuming a dispersive wave at frequency $\omega_{0}+\delta \omega$, where $\omega_{0}$ is the soliton central carrier frequency, is to develop on consecutive round trips, and recalling the Taylor expansion of the propagation constant in Eq.(2.33), it is clear that including third order dispersion $\beta_{3}$ will have considerable influence on soliton sideband generation. These effects are investigated in the next chapter. 


\section{Chapter 5}

\section{High Order Effects}

When soliton pulses become shorter than 4-5 ps, it is vital to include higher order nonlinear and dispersive effects $[16,42]$. For the purposes of this investigation the original Kutz model has been extended to include Raman self-frequency shift, self-steepening and third order dispersion. This chapter investigates these effects separately, before incorporating them simultaneously for modeling ultrashort pulse generation.

\subsection{Higher Order Terms}

The CGLE can be extended to include higher order nonlinear and dispersive effects in terms of the soliton units presented in Eq.(2.65) and can be rewritten as:

$$
\begin{aligned}
i \frac{\partial U}{\partial \xi} & +i \Gamma U-\frac{1}{2} \operatorname{sgn}\left(\beta_{2}\right) \frac{\partial^{2} U}{\partial \tau^{2}}-i \delta \frac{\partial^{3} U}{\partial \tau^{3}}=i G(\xi)\left(1+\tau_{\Omega} \frac{\partial^{2}}{\partial \tau^{2}}\right) U \\
& -N^{2}\left[|U|^{2} U+i s \frac{\partial}{\partial \tau}\left(|U|^{2} U\right)-\tau_{R} U \frac{\partial|U|^{2}}{\partial \tau}\right]
\end{aligned}
$$

Where the parameters $\tau_{R}, s$ and $\delta$ govern Raman self-frequency shift, self-steepening and third order dispersion respectively and are defined as [16]:

$$
\tau_{R}=\frac{T_{R}}{T_{0}} \quad s=\frac{1}{\omega_{0} T_{0}} \quad \delta=\frac{\beta_{3}}{6 \beta_{2} T_{0}}
$$


These effects become fully pronounced for femtosecond pulses. As the mode-locked laser system at hand produces pulses as short as 50-70 fs, the values for the higher order terms used in this investigation follow [42] and are $\tau_{R} \approx 0.1, s \approx 0.03$ and $\delta \approx 0.03$ for 50 fs pulses with $T_{R}=3$ fs in standard silica fibers [16]. The following sections investigate each effect separately through bifurcation diagrams. The simulations performed solve Eq.(5.1), Eqs.(2.71) and (2.72) in a self-consistent manner using the variations of the SSFM described in Chapter 3. Essentially, incorporating these higher order terms result in an extended difference $\hat{D}$ and nonlinear $\hat{N}$ operators. Hence, Eqs.(3.2) and (3.3) are now expressed as:

$$
\begin{aligned}
& \hat{D}=-\Gamma-\frac{i}{2} \operatorname{sgn}\left(\beta_{2}\right) \frac{\partial^{2}}{\partial \tau^{2}}+\delta \frac{\partial^{3}}{\partial \tau^{3}}+G(\xi)+G(\xi) \tau_{\Omega} \frac{\partial^{2}}{\partial \tau^{2}} \\
& \hat{N}=i N^{2}\left[|U|^{2}+i \frac{s}{U} \frac{\partial}{\partial \tau}\left(|U|^{2} U\right)-\tau_{R} \frac{\partial|U|^{2}}{\partial \tau}\right]
\end{aligned}
$$

The OOP implementation presented in Chapter 3 enables a straightforward inclusion of these higher order effects. Further, smaller step sizes are required to attain convergent numerical solutions, as the higher order terms involve nonlinear second and third order derivatives, which considerably overload the numerics. In this work, the results were confirmed among all three SSFM variations with smaller step sizes. The simulations are designed for a resonant coupling occurring between $(i=1)$ and $(j=4)$ with $\left(d_{14}=12, c_{11}=c_{44}=0.2, c_{14}=20\right.$ and $\left.\Delta=0\right)$. The normalized length of both fibre and grating is set to $\xi=1$ and $\xi_{G}=0.1$ respectively. The normalized gain parameters are $\tau_{\Omega}=0.1$ corresponding to a gain bandwidth of $\Omega=25 \mathrm{~nm}$ and $E_{s a t}=1$. The fibre loss is assumed to be $0.2 \mathrm{~dB} / \mathrm{km}$ and the output coupler is $5 \%$. In addition, $G_{0}$ was increased to cover the dynamics within the first coexisting attractor with low ASE input pulse. The bifurcation diagrams reported here are a plot of pulse energy over the laser cavity passes from 180 to 200 . 


\subsection{Raman Self-Frequency Shift}

Raman self-frequency shift also known as Raman intrapulse scattering is the most significant among the higher order nonlinear effects and has been observed numerically [42] and experimentally [101]. The origin of the self-frequency shift is the broad spectrum of ultrashort pulses, where Raman gain transfers energy from high to low frequency components of the same pulse. The effect on pulse propagation is a temporal walk-off and a low intensity pulse appearing on the leading edge of the incident pulse $[16,42]$. To investigate the dynamics of the laser under the effect of the Raman intrapulse scattering all parameters were fixed, while the normalized saturated gain $G_{0}$ was increased from 1 to 3.0 corresponding to an amplification range of 5-17 dB with low ASE input. This range of amplification corresponds to the first coexisting attractor, where the pulse train exhibits period one, two and four [46]. Similar to the previous simulations, the pulse energy is plotted against the linearly increasing normalized saturated gain $G_{0}$. To isolate the effect of Raman self-frequency shift $\tau_{R}=0.1$, while both $\delta$ and $s$ were set to zero in Eq.(5.1). The resulting bifurcation diagram is shown in Fig. 5.1 (a). Including the Raman term, results in highly unstable and irregular pulse energy in the first segment of increased gain after which the soliton pulse energy exhibit period tripling. The general behavior of the laser dynamics change abruptly at $G_{0}=1.6$, which corresponds to the bifurcation point in Fig. 4.5 (a), when the Raman term was excluded. In an attempt to better understand the previous results, a simulation was performed at a specific cavity gain value of $G_{0}$ $=1.0$ with $\tau_{R}=0.1$. The pulse energy plotted against laser passes for this simulation is shown in Fig. 5.1 (b). Normally, the laser pulse would mode-lock with a stable energy after about 80 cavity passes. However, as the Raman term is included the mode-locked pulse energy experiences an abrupt decrease every 10 passes, before it increases again reaching an irregular peak value. The maximum reachable energy 


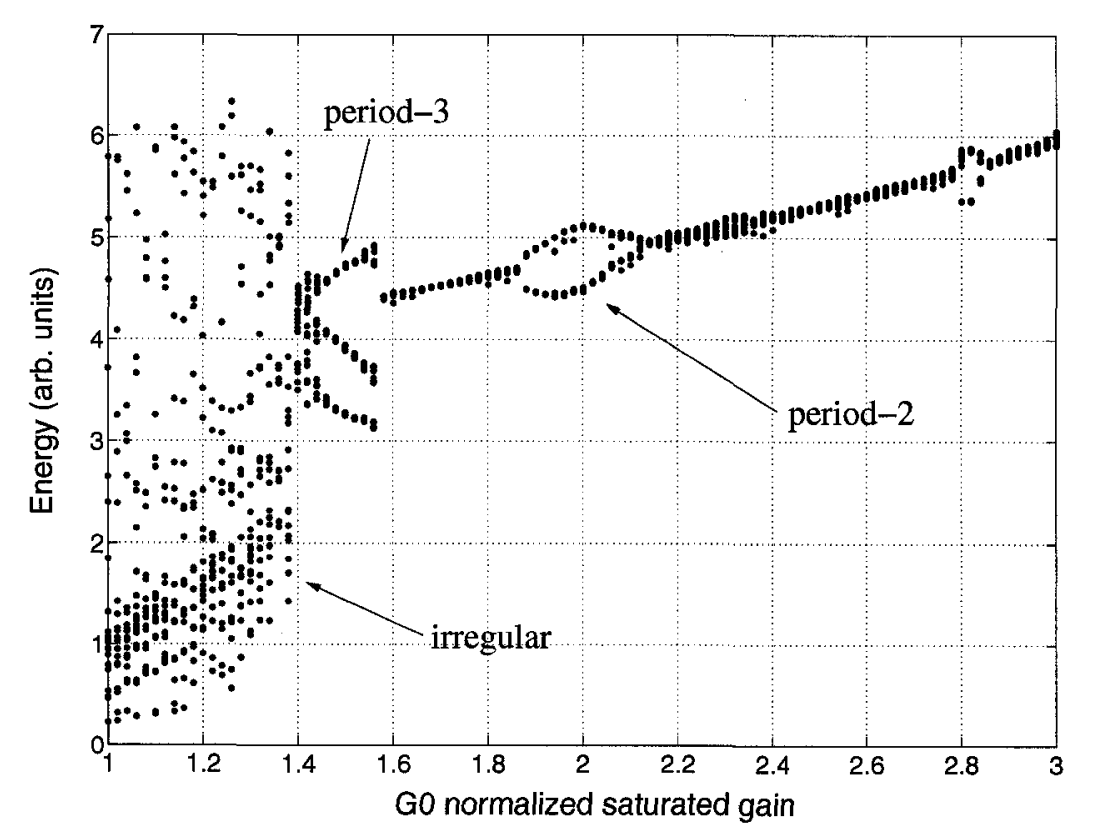

(a)

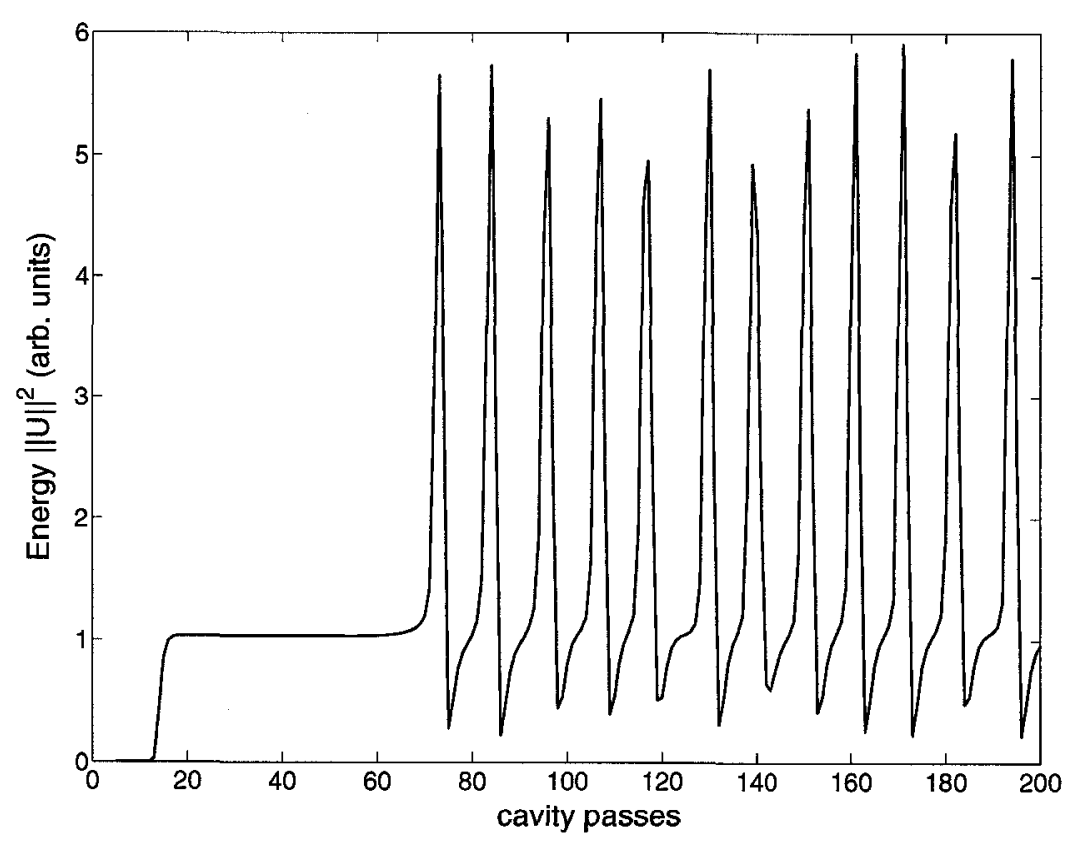

(b)

Figure 5.1: Laser dynamics with $\tau_{R}=0.1$ and low ASE input (a) bifurcation diagram of pulse energy $E$ plotted against the normalized saturated gain for $G_{0}=1$ to 3 (b) pulse energy $E$ plotted against laser passes at $G_{0}=1.0$ 
is higher when the Raman term is excluded, this is expected as the Raman term serves as a non-Hamiltonian perturbation to the CGLE, hence, pulse energy is not preserved [16]. Physically, the energy is dissipated through stimulating molecular vibrations [38]. The behavior seen in Fig. 5.1 (b) is a consequence of the pulse-shaping mechanism introduced by the LPFG. As the laser mode-locks a narrow pulse is generated, with a spectral width large enough for the fiber Raman gain to transfer energy from high to low frequency spectral components [15]. This does not only reduce the total pulse energy, but also results in a smaller side pulse generation in the temporal window. However, in this laser system, the LPFG acts as an intensity discriminator, attenuating low intensity portions of the pulse. This explains the abrupt decrease in pulse energy every 10 passes when the Raman term is included as the LPFG filters the already low energy pulse restarting the mode-locking mechanism.

\section{$5.3 \quad$ Self-Steepening}

The phenomenon of self-steepening also known as optical shock arises from the intensity dependence of the group velocity, which creates an optical shock on the trailing edge of the pulse $[16,42]$. In essence, the peak of the pulse moves slower than its wings. Temporally, this group velocity mismatch within the pulse is manifested as a walkoff. Mathematically, the shock term arises from the inclusion of the first derivative of the general form of $P_{N L}$ [16] in the derivation of the NLSE presented in Chapter 2. To investigate the effects of self-steepening, a simulation was performed with the saturated gain linearly increasing from 1.0-3.0, with low ASE input. The shock term was set to $s=0.03$, while both Raman intrapulse scattering and third order dispersion were set to zero in Eq.(5.1). The resulting bifurcation diagram is shown in Fig. 5.2 (a). It is clear that the bifurcation diagram of Fig. 5.2 (a) is not remarkably different than that of Fig. 4.5 (a), where the shock term was normally excluded. 


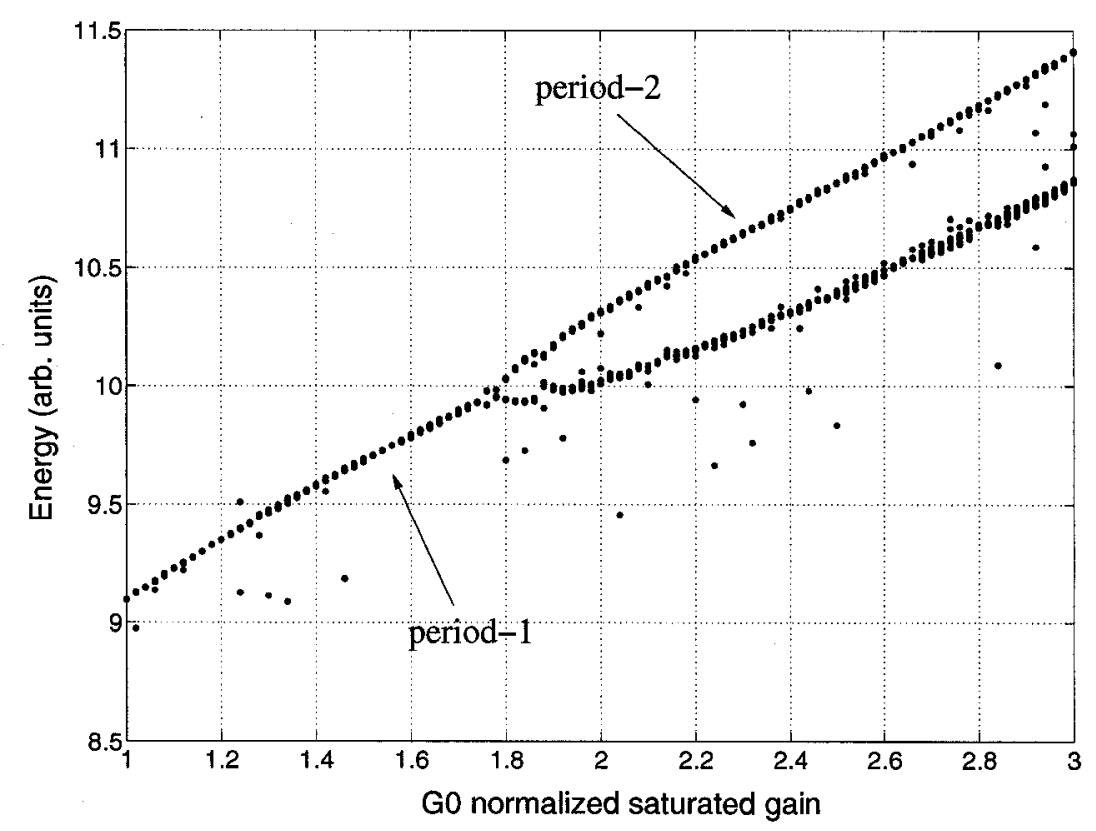

(a)

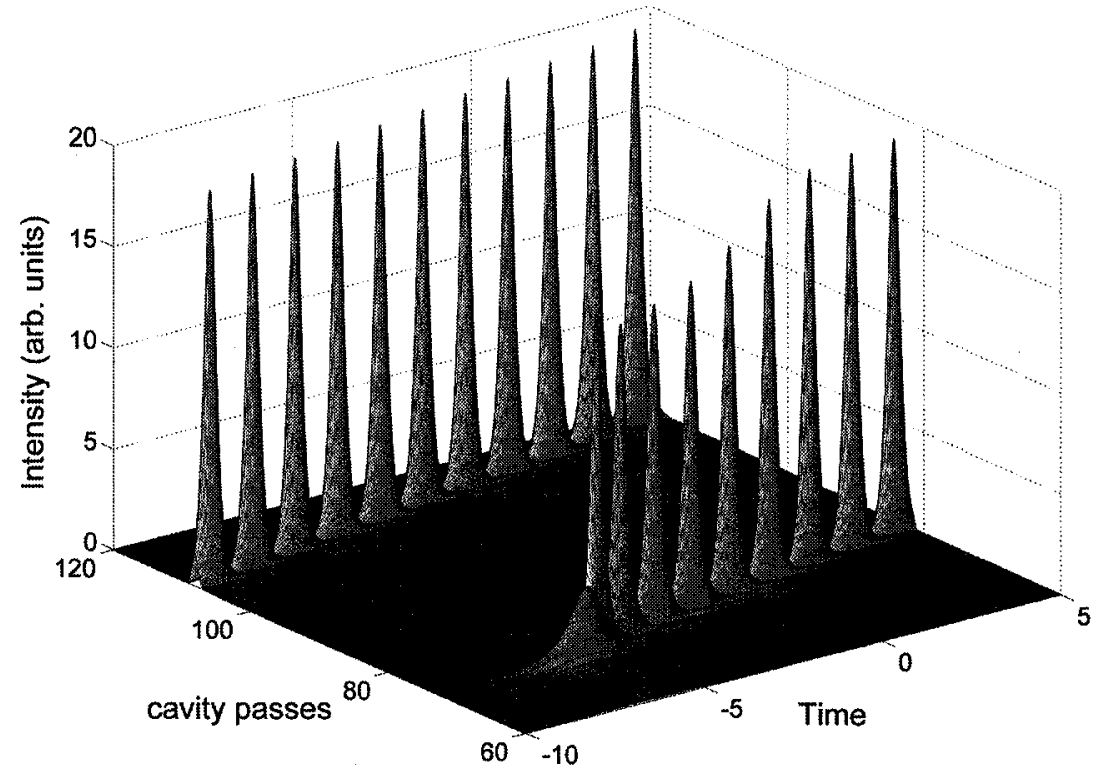

(b)

Figure 5.2: Laser dynamics with $s=0.03$ and low ASE input (a) bifurcation diagram of pulse energy $E$ plotted against the normalized saturated gain for $G_{0}=1$ to 3 (b) pulse intensity profile plotted against laser passes at $G_{0}=1.0$ 
This is expected as the shock effect results in a temporal walk-off with no significant energy alteration, as oppose to non-Hamiltonian perturbations, as the case with Raman intrapulse scattering. In an attempt to observe this temporal walk-off, a simulation was performed at a specific cavity gain value of $G_{0}=1.0$ the evolution profile of the pulse intensity plotted against the cavity round trips shown in Fig. 5.2 (b). The intensity dependent nature of $v_{g}$ forms a side pulse, which is rapidly dissipated. This smaller soliton-like pulse is not seen in Fig. 5.2 (b) as the LPFG filters it out. However, the shock effect is manifested as a shift in the pulse centre.

\subsection{Third Order Dispersion}

Dispersion causes optical pulse broadening, which is usually dominated by the term $\beta_{2}$. However, for some cases it is necessary to include third order dispersion $\beta_{3}$ [102]. These cases include propagation near the zero-dispersion wavelength, where $\beta_{2} \approx 0$ and the effect of $\beta_{3}$ is fully pronounced [16]. Further, for ultrashort pulses propagation it is essential to include $\beta_{3}$ even though $\beta_{2} \neq 0$ [42]. This is a consequence of the parameter $\Delta \omega / \omega_{0}$ being large enough rendering the assumption made for truncating the Taylor expansion of $\beta$ in Eq.(2.33) invalid [16,42]. To investigate the dynamics of the laser under the effect of the third order dispersion all parameters were fixed, while the normalized saturated gain $G_{0}$ was increased from 1 to 3.0 with low ASE input. This range of amplification corresponds to the first coexisting attractor, where the pulse train exhibits period one, two and four [46]. Similar to the previous simulations, the pulse energy is plotted against the linearly increasing normalized saturated gain. To isolate the effect of third order dispersion $\delta=0.03$, while both $\tau_{R}$ and $s$ were set to zero in Eq.(5.1). The resulting bifurcation diagram is shown in Fig. 5.3 (a). It is clear that the bifurcation diagram of Fig. 5.3 (a) is more stable than that resulting when the Raman or shock effects are included. The bifurcation point is further delayed than 


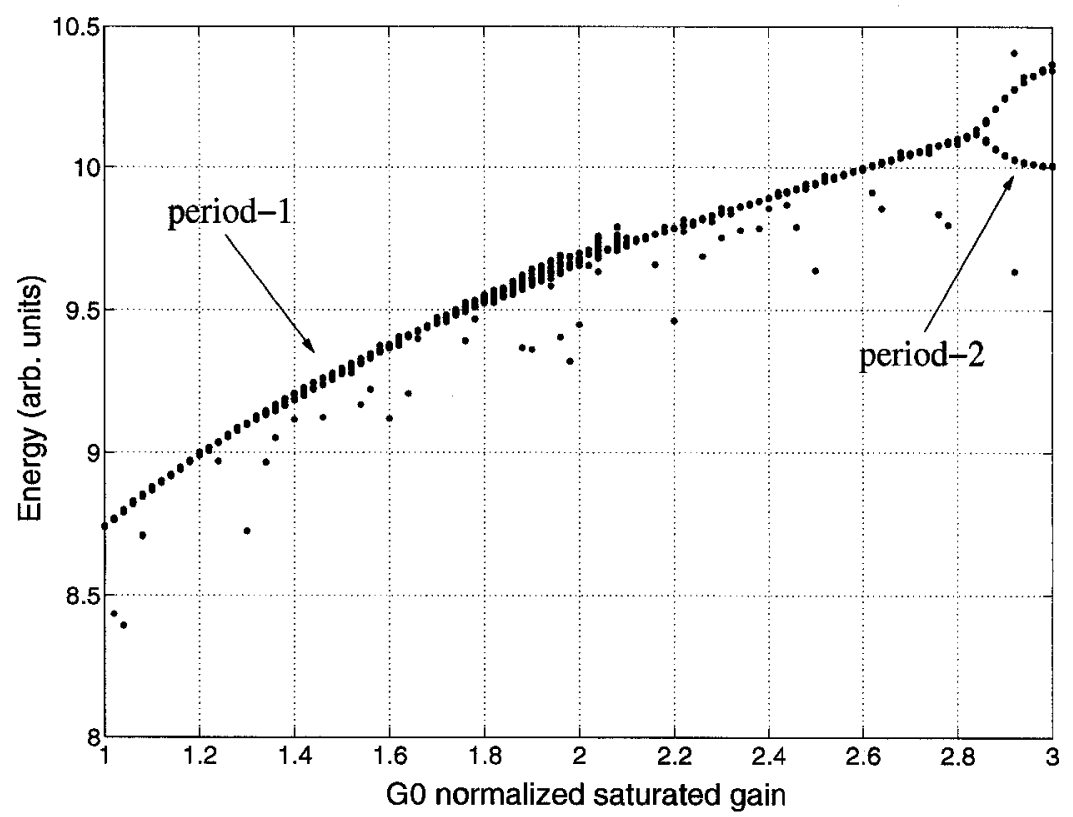

(a)

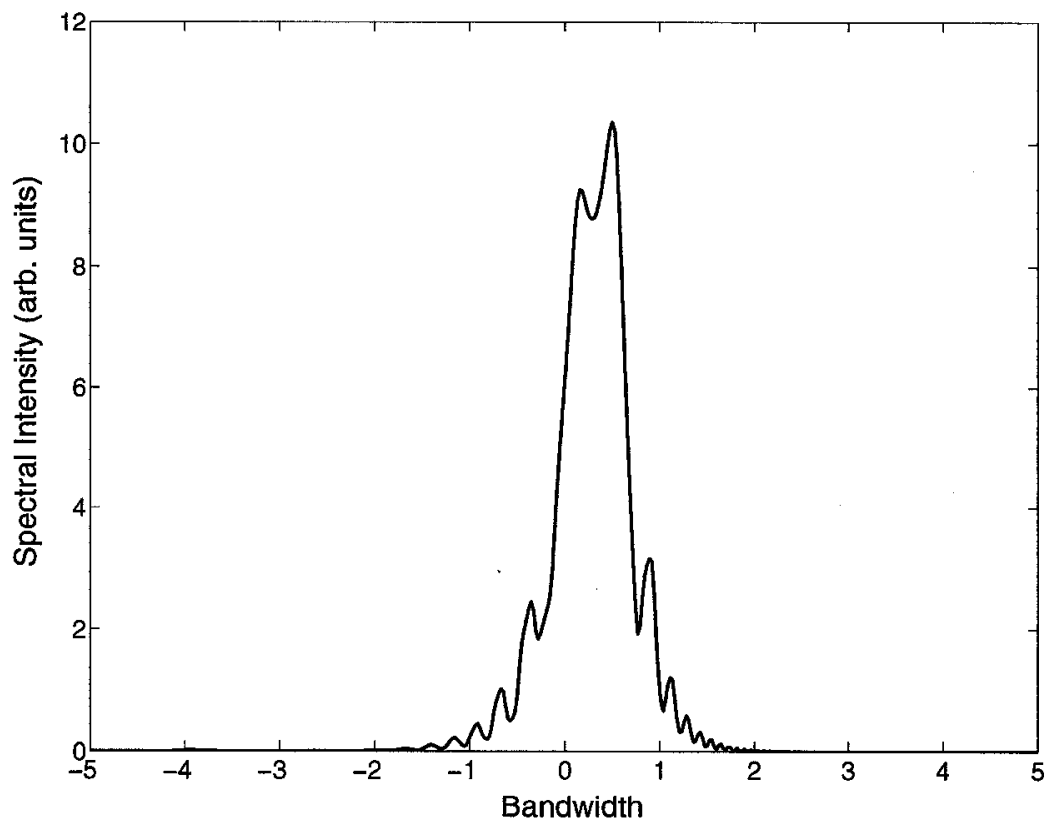

(b)

Figure 5.3: Laser dynamics with $\delta=0.03$ and low ASE input (a) bifurcation diagram of pulse energy $E$ plotted against the normalized saturated gain for $G_{0}=1$ to 3 (b) pulse asymmetric spectral intensity at $G_{0}=1.0$ 
that of the shock term and appears at $G_{0}=2.84$. The effect of third order dispersion is to distort the pulse such that it becomes asymmetric with an oscillatory structure near one of its edges [15]. As we are operating with a positive $\beta_{3}$, the oscillation occurs on the trailing edge of the pulse [16]. The pulse asymmetry is clearer in the frequency domain as shown in Fig. 5.3 (b). It is also clear that including third order dispersion has a considerable influence on the soliton sideband generation reported in Fig. 4.16. The previous results show more stability when third order dispersion is included. This observation may prove promosing, as PCFs have a tailorable dispersion slope, which can be designed to eliminate any present instability [100].

\subsection{Ultrashort Pulse Generation}

Mode-locked lasers resonate to produce ultrashort pulses with a FWHM in the femtosecond range. This requires the inclusion of all higher order dispersive and nonlinear effects. However, for pulses shorter than 10 fs the SVEA itself is under question [16]. The evolution of ultrashort pulses in the laser cavity can studied by solving Eq.(5.1), Eqs.(2.71) and (2.72) numerically. As an example, a simulation was performed with all cavity parameters fixed, while the normalized saturated gain $G_{0}$ was increased from 1 to 3.0 with low ASE input. Further, the values for the higher order terms used were $\tau_{R}=0.1, s=0.03$ and $\delta=0.03$ [16]. The resulting bifurcation diagram is shown in Fig. 5.4. The bifurcation diagram shows a rapid energy jump at a cavity gain value of $G_{0}=2.12$ to an unstable and chaotic coexisting attractor with an average energy level of approximately 8 arbitrary units. The combination of Raman self-frequency shift, self-steepening and third order dispersion result in a phenomena known as soliton decay [42]. The process of solitons decay results in the splitting of higher-order solitons into their constituents [15]. However, the effect of soliton decay on solitary-waves is beyond the scope of this study and requires further investigation. 


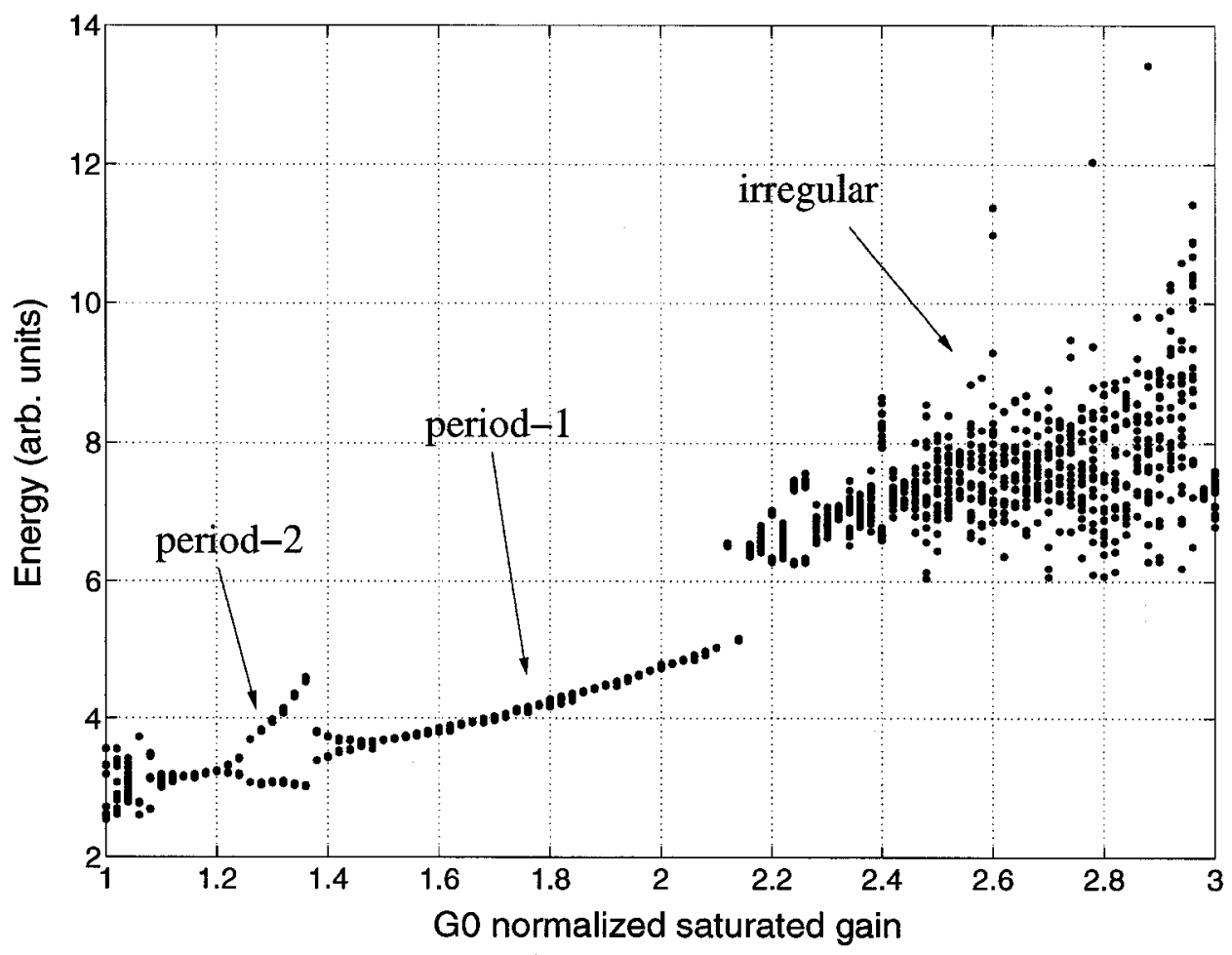

Figure 5.4: Bifurcation diagram of pulse energy $E$ plotted against the normalized saturated gain for $G_{0}=1$ to 3 with low ASE input, $\tau_{R}=0.1, s=0.03$ and $\delta=0.03$ 


\section{Chapter 6}

\section{Conclusion}

This thesis explored the nonlinear dynamics of a passive mode-locked fiber laser containing a LPFG. The numerical model was based on the CGLE and the nonlinear coupled mode equations of the LPFG. An object oriented approach was adapted in programming the laser system. Object oriented concepts, such as polymorphism, encapsulation, operator overload and delegation were used to implement extendable and reusable $\mathrm{C}++$ code for scientific computing. It was found that the level of encapsulation is inversely proportional to the computational time. To accurately capture the wide range of dynamics, robust and numerically stable variations of the split-step Fourier method were implemented.

The numerical results show the existence of self-starting, autosolitons in the cavity of the laser with both single and bound soliton pulse trains exhibiting period doubling bifurcations and eventual route to chaos as the normalized saturated gain is increased. Furthermore, long period pulsation, soliton sidebands and coexisting attractors excited by multisoliton formation and soliton energy quantization are present. The nonlinear dynamics of the laser are presented through plotting the output pulse energy against the linearly increasing gain so obtaining bifurcation diagrams.

In most work on passive mode-locked lasers, one master equation is used to model the system dynamics. However, in this work the CGLE, as it stands alone, has 
no phenomenological intensity discrimination term and hence does not enable modelocking. It is the combination of the CGLE and the nonlinear mode coupling equations of the LPFG that allow the laser model to mode-lock. Thus, the wide range of dynamics observed is an intrinsic property of the soliton laser system regardless of the cavity model used.

The numerical investigation was further extended through the inclusion of higher order nonlinear and dispersive effects, such as Raman self-frequency shift, selfsteepening and third order dispersion. These phenomena affect the laser dynamics resulting in different regions of instability in the bifurcation diagrams.

Future work would be to closely examine the effect of third order dispersion on the dynamics of the laser. As including third order dispersion resulted in a more stable bifurcation diagram. This opens the possibility of using a PCF in the laser cavity. A PCF laser cavity would not only solve the high nonlinear index problem for the LPFG, but also have a tailorable dispersion slope, allowing greater control over third order dispersion for the purpose of locking one of the coexisting attractors and improving the performance.

Furthermore, the effect of inserting the LPFG as a pulse shaping element in more sophisticated laser cavity designs, such as the figure of eight laser, in hope of attaining shorter pulses, would be of interest. 


\section{List of References}

[1] E. Desurvire, Erbium-Doped Fiber Amplifiers : principles and applications. New York: Wiley-Interscience, 2002.

[2] I. N. Duling, Compact Sources of Ultrashort Pulses. Cambridge: Cambridge University Press, 1995.

[3] O. Svelto, Principles of Lasers. New York: Plenum Press, 1998.

[4] E. Snitzer, "Optical maser action of $N d^{+3}$ in a barium crown glass," Phys. Rev. Lett., vol. 7, pp. 444-446, 1961.

[5] J. Stone and C. A. Burrus, "Neodymium-doped silica lasers in end-pumped fiber geometry," Appl. Phys. Lett., vol. 23, pp. 388-389, 1973.

[6] R. J. Mears, L. Reekie, S. B. Poole, and D. N. Payne, "Neodymium-doped silica single-mode fibre lasers," Electron. Lett., vol. 21, pp. 738-740, 1985.

[7] W. E. Lamb, "Theory of an optical maser," Phys. Rev., vol. 134, pp. 1429-1450, 1964.

[8] M. I. Dzhibladze, Z. G. Esiashvili, E. S. Teplitskii, S. K. Isaev, and V. R. Sagaradze, "Mode locking in a fiber laser," Soviet Journal of Quantum Electronics, vol. 10, pp. 432-434, 1983.

[9] H. A. Haus, "Mode-locking of lasers," IEEE J. Sel. Top. Quantum Electron., vol. 6, pp. 1173-1185, 2000.

[10] V. J. Matsas, D. J. Richardson, T. P. Newson, and D. N. Payne, "Characterization of a self-starting, passively mode-locked fiber ring laser that exploits nonlinear polarization evolution," Opt. Lett., vol. 18, pp. 358-360, 1993.

[11] W. Kaiser, Ultrashort laser pulses: generation and applications. Berlin: Springer-Verlag, 1993. 
[12] K. Okamoto, Fundamentals of optical waveguides. San Diego: Academic Press, 2000 .

[13] I. N. Duling, "All-fiber ring soliton laser mode locked with a nonlinear mirror," Opt. Lett., vol. 16, pp. 539-541, 1991.

[14] L. F. Mollenauer and R. H. Stolen, "The soliton laser," Opt. Lett., vol. 9, pp. 13-15, 1984.

[15] Y. S. Kivshar and G. P. Agrawal, Optical Solitons: From Fibers to Photonic Crystals. New York: Academic Press, 2003.

[16] G. P. Agrawal, Nonlinear Fiber Optics. New York: Academic Press, 2001.

[17] A. Hasegawa and F. Tappert, "Transmission of stationary nonlinear optical pulses in dispersive dielectric fibers. i. anomalous dispersion," Appl. Phys. Lett., vol. 23, pp. 142-144, 1973.

[18] L. F. Mollenauer, R. H. Stolen, and J. P. Gordon, "Experimental observation of picosecond pulse narrowing and solitons in optical fibers," Phys. Rev. Lett., vol. 45, pp. 1095-1098, 1980.

[19] V.S. Grigoryan and T. S. Muradyan, "Evolution of light pulses into autosolitons in nonlinear amplifying media," J. Opt. Soc. Amer. B, vol. 8, pp. 1757-1765, 1991.

[20] N. G. Usechak, G. P. Agrawal, and J. D. Zuege, "FM mode-locked fiber lasers operating in the autosoliton regime," IEEE J. Quantum Electron., vol. 41, pp. $753-761,2005$.

[21] J. M. Soto-Crespo, M. Grapinet, P. Grelu, and N. Akhmediev, "Bifurcation and multiple-period soliton pulsation in a passively mode-locked fiber laser," Phys. Rev. E, vol. 70, p. 066612, 2004.

[22] L. M. Zhao, D. Y. Tang, and A. Q. Liu, "Chaotic dynamics of a passively mode-locked soliton fiber ring laser," Chaos, vol. 16, p. 013128, 2006.

[23] K. Tamura, C. R. Doerr, H. A. Haus, and E. P. Ippen, "Soliton fiber ring laser stabilization and tuning with a broad interactivity filter," IEEE Photon. Technol. Lett., vol. 6, pp. 697-699, 1994.

[24] A. B. Grudinn, D. J. Richardson, and D. N. Payne, "Energy quantization in figure eight fiber laser," Elec. Lett., vol. 28, pp. 67-68, 1992. 
[25] D. Y. Tang, L. M. Zhao, B. Zhao, and A. Q. Liu, "Mechanism of multisoliton formation and soliton energy quantization in passively mode-locked fiber lasers," Phys. Rev. A, vol. 72, p. 043816, 2005.

[26] W. Szemplinacuteska-Stupnicka, A. Zubrzycki, and E. Tyrkiel, "Properties of chaotic and regular boundary crisis in dissipative driven nonlinear oscillators," Nonlinear Dynamics, vol. 19, pp. 19-36, 1999.

[27] V. N. Chizhevsky, "Coexisting attractors in a $\mathrm{CO}_{2}$ laser with modulated losses," J. Opt. B : Quantum Semiclass. Opt., vol. 2, pp. 711-717, 2000.

[28] J. M. Saucedo-Solorio, A. N. Pisarchik, A. V. Kir'yanov, and V. Aboite, "Generalized multistability in a fiber laser with modulated losses," J. Opt. Soc. Amer. $B$, vol. 20, pp. 490-496, 2003.

[29] A. N. Pisarchik, Y. O. Barmenkov, and A. V. Kiryano, "Experimental characterization of the bifurcation structure in an erbium-doped fiber laser with pump modulation," IEEE J. Quantum Electron., vol. 39, pp. 1567-1571, 2003.

[30] L. Luo, T. J. Tee, and P. L. Chu, "Chaotic behavior in erbium-doped fiber-ring laser," J. Opt. Soc. Amer. B, vol. 15, pp. 972-978, 1998.

[31] R. Hergenroder, O. Samek, and V. Hommes, "Femtosecond laser ablation elemental mass spectrometry," Mass Spectrom. Rev., vol. 25, pp. 551-572, 2006.

[32] K. Intrachat and J. N. Kutz, "Theory and simulation of passive mode-locking dynamics using a long-period fiber grating," IEEE J. Quantum Electron., vol. 39, pp. 1572-1578, 2003.

[33] MathWorks, MATLAB. United States: wwww.mathworks.com, 2007.

[34] D. Yang, C++ and Object-Oriented Numeric Computing for Scientists and Engineers. New York: Springer-Verlag, 2001.

[35] J. N. Kutz, B. Eggleton, J. Stark, and R. Slusher, "Nonlinear pulse propagation in long-period fiber gratings: theory and experiment," IEEE J. Select. Topics Quantum Electron., vol. 5, pp. 1232-1245, 1997.

[36] M. Born and E. Wolf, Principles of Optics. Cambridge: Cambridge University Press, 1999.

[37] K. Kawano and T. Kitoh, Introduction to Optical Waveguide Analysis: Solving Maxwell's Equation and the Schrödinger Equation. New York: WileyInterscience, 2001. 
[38] R. W. Boyd, Nonlinear Optics. San Diego, CA: Academic Press, 1992.

[39] G. P. Agrawal, Fiber-Optic Communication Systems. New York: WileyInterscience, 2002.

[40] G. P. Agrawal, "Optical pulse propagation in doped fiber amplifiers," Phys. Rev. A, vol. 44, pp. 7493-7501, 1991.

[41] P. W. Milonni and J. H. Eberly, Lasers. New York: Wiley-Interscience, 1988.

[42] G. P. Agrawal, Applications of Nonlinear Fiber Optics. San Diego, CA: Academic Press, 2001.

[43] H. A. Haus, J. G. Fujimoto, and E. P. Ippen, "Structures for additive pulse mode locking," J. Opt. Soc. Am. B, vol. 8, pp. 2068-2076, 1991.

[44] V. E. Zakharov and A. B. Shabat, "Exact theory of two-dimension self-focusing and one-dimension self-modulation of waves in nonlinear media," Soviet Phys. JETP, vol. 34, pp. 62-69, 1972.

[45] A. A. N. Akhmediev and J. M. Soto-Cresp, "Multisoliton solutions of the complex Ginzburg-Landau equation," Phys. Rev. Lett., vol. 79, pp. 4047-4051, 1997.

[46] A. S. Karar, A. L. Steele, and T. Smy, "Nonlinear dynamics of a passively modelocked fiber laser containing a long-period fiber grating," submitted to IEEE J. Quantum Electron., vol. JQE-131029, 2007.

[47] J. Proctor and J. N. Kutz, "Nonlinear mode-coupling for passive mode-locking: application of waveguide arrays, dual-core fibers and/or fiber arrays," Opt. Express, vol. 13, pp. 8933-8950, 2005.

[48] C. R. Giles and E. Desurvire, "Modeling erbium-doped fiber amplifiers," J. Lightwave Technol, vol. 9, pp. 271-283, 1991.

[49] A. Hasegawa and Y. Kodama, "Guiding-center soliton in optical fibers," Opt. Lett., vol. 15, pp. 1443-1445, 1990.

[50] A. Othonos and K. Kalli, Fiber Bragg Gratings: Fundamentals and Applications in Telecommunications and Sensing. Norwood, MA: Artech House Publishers, 1999 .

[51] T. Erdogan, "Cladding-mode resonances in short- and long-period fiber grating filters," J. Opt. Soc. Am. A, vol. 14, pp. 1760-1773, 1997. 
[52] A. M. Vengsarkar, P. J. Lemaire, J. B. Judkins, V. Bhatia, T. Erdogan, and J. E. Sipe, "Long-period fiber gratings as band-rejection filters," J. Lightwave Technol., vol. 14, pp. 58-65, 1996.

[53] Optiwave, OptiGrating. Ottawa: www.optiwave.com, 2006.

[54] S. M. Jensen, "The nonlinear coherent coupler," IEEE J. Quantum Electron., vol. 18, pp. 1580-1583, 1982.

[55] SMF-28, Single-Mode Optical Fiber. New York: Corning, 2002.

[56] B. J. Eggleton, R. E. Slusher, J. B. Judkins, J. B. Stark, and A. M. Vengsarkar, "All-optical switching in long-period fiber gratings," Opt. Lett., vol. 22, pp. 883$885,1997$.

[57] Y. Jeong and B. Lee, "Nonlinear property analysis of long-period fiber gratings using discretized coupled-mode theory," IEEE J. Quantum Electron., vol. 35, pp. 1284-1292, 1999.

[58] J. C. Knight, T. A. Birks, P. S. J. Russell, and D. M. Atkin, "All-silica singlemode optical fiber with photonic crystal cladding," Opt. Lett., vol. 21, pp. 15471549, 1996.

[59] A. Zhang, H. Liu, M. S. Demokan, and H. Y. Tam, "Stable and broad bandwidth multiwavelength fiber ring laser incoporating a highly nonlinear photonic crystal fiber," IEEE Photon. Technol. Lett., vol. 12, pp. 2535-2537, 2005.

[60] Y. Zhu, P. Shum, M. R. H. Chong, and C. Lu, "Strong resonance and a highly compact longperiod grating in a large-mode-area photonic crystal fiber," Opt. Express, vol. 11, pp. 1900-1905, 2003.

[61] L. Rindorf, J. B. Jensen, M. Dufva, L. H. Pedersen, P. E. Hoiby, and O. Bang, "Photonic crystal fiber long-period grating for biochemical sensing," Opt. Express, vol. 14, pp. 8224-8231, 2006.

[62] M. Asobe, "Nonlinear optical properties of chalcogenide glass fibers and their application to all-optical switching," Opt. Fiber Technol., vol. 3, pp. 142-148, 1997.

[63] M. Asobe, T. Kanamori, and K. Kubodera, "Applications of highly nonlinear chalcogenide glass fibers inultrafast all-optical switches," IEEE J. Quantum Electron., vol. 29, pp. 2325-2333, 1993. 
[64] H. C. Nguyen, K. Finsterbusch, D. J. Moss, and B. J. Eggleton, "Dispersion in nonlinear figure of merit of $\mathrm{As}_{2} \mathrm{Se}_{3}$ chalcogenide fibre," Electron. Lett., vol. 42, pp. 571-572, 2006.

[65] L. B. Fu, M. Rochette, V. G. Ta'eed, D. J. Moss, and B. J. Eggleton, "Investigation of self-phase modulation based optical regeneration in single mode $\mathrm{As}_{2} \mathrm{Se}_{3}$ chalcogenide glass fiber," Opt. Express, vol. 13, pp. 7637-7644, 2005.

[66] D. Pudo, E. C. Mägi, and B. J. Eggleton, "Long-period gratings in chalcogenide fibers," Opt. Express, vol. 14, pp. 3763-3766, 2006.

[67] M. J. Ablowitz and H. Segur, Soliton and the Inverse Scattering Transform. Philadelphia: SIAM, 1981.

[68] Q. Chang, E. Jia, and W. Suny, "Difference schemes for solving the generalized nonlinear Schrödinger equation," J. Comp. Phys., vol. 148, pp. 397-415, 1999.

[69] R. A. Fisher and W. K. Bischel, "Numerical studies of the interplay between self-phase modulation and dispersion for intense plane-wave laser pulses," $J$. Appl. Phys., vol. 46, pp. 4921-4934, 1975.

[70] T. R. Taha and M. J. Ablowitz, "Analytical and numerical aspects of certain nonlinear evolution equations part II: numerical nonlinear Schrödinger equation," J. Comp. Phys., vol. 55, pp. 203-230, 1984.

[71] X. Liu and B. Lee, "A fast method for nonlinear Schrödinger equation," IEEE Photon. Technol. Lett., vol. 15, pp. 1549-1551, 2003.

[72] W. H. Press, S. A. Teukolsky, W. T. Vetterling, and B. P. Flannery, Numerical Recipes in FORTRAN. New York: Cambridge University Press, 1992.

[73] O. V. Sinkin, R. Holzlohner, J. Zweck, and C. R. Menyuk, "Optimization of the split-step fourier method in modeling optical-fiber communications systems," $J$. Lightwave Technol., vol. 21, pp. 61-68, 2003.

[74] G. Buzzi-Ferraris, Scientific C++ Building Numerical Libraries the ObjectOriented Way. Reading, Mass.: Addison-Wesley, 1993.

[75] D. Yevick, A first Course in Computational Physics and Object-Oriented Programming with $\mathrm{C}++$. Cambridge: Cambridge University Press, 2005.

[76] G. Booch, "Object-oriented development," IEEE Trans. Soft. Eng., vol. 12, pp. 211-221, 1986. 
[77] A. Eliens, Principles of Object-Oriented Software Development. Reading, Mass.: Addison-Wesley, 1994.

[78] M. L. Zimmerman and P. Mallasch, "Using object-oriented programming in computational electromagnetic codes," Antennas and Propagation Society International Symposium, vol. 2, pp. 1099-1102, 1995.

[79] F. Ladouceur and L. Poladian, "Object oriented numerical modelling for integrated optics," in Physics and simulation of optoelectronic devices VI (P. B. Marek Osinski and A. Ishibashi, eds.), vol. 3283 of Proc. SPIE, pp. 765-771, 1998.

[80] K. Gallivan and B. Marsolf, "Practical issues related to developing objectoriented numerical libraries," In OON-SKI'94 Proceedings of the Second Annual ObjectOriented Numerics Conference, pp. 93-106, 1994.

[81] J. Weidendorfer, KCachegrind and Calltree. General Public License, 2003. http://kcachegrind.sourceforge.net/cgi-bin/show.cgi.

[82] N. Dale, C. Weems, and M. Headington, Programming and Problem Solving with $\mathrm{C}++$. London: Jones and Bartlett, 2000.

[83] T. L. Veldhuizen, "Scientific computing: C++ versus Fortran: C++ has more than caught up," Dr. Dobb's Journal of Software Tools, vol. 22, pp. 36-38, 1997.

[84] G. Csányi, S. Winfield, J. Kermode, M. Payne, A. Comisso, A. D. Vita, and N. Berstein, "Expressive programming for computational physics in Fortran 95+," IOP Comp. Phys. Group, pp. 1-11, 2007.

[85] K. Ikeda, "Multiple-valued stationary state and its instability of the transmitted light by a ring cavity system," Opt. Comm., vol. 137, pp. 136-142, 1997.

[86] D. Y. Shen, J. K. Sahu, and W. A. Clarkson, "Highly efficient Er,Yb-doped fiber laser with $188 \mathrm{~W}$ free-running and $>100 \mathrm{~W}$ tunable output power," Opt. Express, vol. 13, pp. 4916-4921, 2005.

[87] D. Y. Tang and L. M. Zhao, "Generation of 47-fs pulses directly from an erbiumdoped fiber laser," Opt. Lett., vol. 32, pp. 41-43, 2007.

[88] A. Gordon, O. Gat, B. Fisher, and F. X. Kartner, "Self-starting of passive mode locking," Opt. Express, vol. 14, pp. 11142-11154, 2006. 
[89] D. Abraham, R. Nagar, and G. Eisenstein, "Self-starting low-noise fiber soliton laser," Opt. Lett., vol. 18, pp. 1508-1510, 1993.

[90] E. Marti-Panameno and V. N. Serkin, "Autosoliton fiber laser," CLEO PACIFIC-RIM, vol. P113, pp. 220-221, 1997.

[91] N. R. Pereira and L. Stenflo, "Nonlinear Schrödinger equation including growth and damping," Phys. Fluids, vol. 20, pp. 1733-1734, 1977.

[92] R. C. Hilborn, Chaos and Nonlinear Dynamics. New York: Oxford University Press, 1994.

[93] A. L. Steele, S. Lynch, and J. E. Hoad, "Stability analysis of nonlinear optical resonators," Chaos, Solitons and Fractals, vol. 9, pp. 935-946, 1998.

[94] F. Sanchez, P. L. Boudec, P. François, and G. Stephan, "Effects of ion pairs on the dynamics of erbium-doped fiber lasers," Phys. Rev. A, vol. 48, pp. 2220 $2229,1993$.

[95] G. Tartwijk and G. Agrawal, "Laser instabilities: a modern perspective," Prog. Quant. Electron., vol. 22, pp. 43-122, 1998.

[96] K. Cuomo and A. Oppenheim, "Circuit implementation of synchronized chaos with applications to communication," Phys. Rev. Lett., vol. 71, pp. 65-68, 1993.

[97] L. Pecora and T. Carrol, "Synchronization of chaotic systems," Phys. Rev. Lett., vol. 64, pp. 821-823, 1990.

[98] G. D. VanWiggeren and R. Roy, "Chaotic communication using time-delayed optical systems," Int. J. Bifur. Chaos, vol. 9, pp. 2129-2156, 1999.

[99] L. G. Luo, P. L. Chu, and H. F. Liu, "1-GHz optical communication system using chaos in erbium-dopedfiber lasers," IEEE Photon. Techol. Lett., vol. 12, pp. $269-271,2000$.

[100] Y. Logvin and H. Anis, "Dynamic regime of Yb-doped fiber laser under the influence of third order dispersion," to be published in SPIE Proc., pp. FLA-6$1-4,2007$.

[101] E. M. Dianov, A. Y. Karasik, P. V. Mamyshev, A. M. Prokhorov, V. N. Serkin, M. F. Stelmakh, and A. A. Fomichev, "Stimulated Raman conversion of multisoliton pulses in quartz optical fibers," JETP Lett., vol. 41, p. 294297, 1985. 
[102] G. P. Agrawal and M. J. Potasek, "Nonlinear pulse distortion in single-mode optical fibers at the zero-dispersion wavelength," Phys. Rev. A, vol. 33, pp. 1765$1776,1986$. 


\section{Appendix A}

\section{$\mathrm{C}++$ Code}

The main function is the only $\mathrm{C}++$ source code shown. Class definitions and functions implementations have been omitted for the sake of conciseness. 
// Simulation input file

$\begin{array}{ll}\text { beta2 }= & -1 \\ \text { segma }= & 0.00 \\ \text { NL_gamma }= & 1 \\ \text { loss_gamma = } & 1.94 \mathrm{e}-5 \\ \text { s = } & 0.00 \\ \text { T_Raman }= & 0.00 \\ \text { tau = } & 0.1 \\ \text { Esat = } & 1.0 \\ \text { G0 = } & 1.0 \\ \mathrm{~N}= & 1 \mathrm{e}-12 \\ \mathrm{~h}= & 0.005 \\ \text { fiberLength }= & 1 \\ \text { T0 = } & 1 \\ \text { numTimeSamples }= & 2048 \\ \text { timeWindow = } & 50 \\ \text { Input Signal } & \text { GAUN } \\ \text { C = } & 0 \\ \text { m }= & 0 \\ \text { ErrorVector } & 000 \\ \text { Tolerance = } & 0 \\ \text { Simulation } & \text { SSSFM } \\ \text { delta = } & 0 \\ \text { d14 = } & 12 \\ \text { c11 = } & 0.2 \\ \text { c44 = } & 0.2 \\ \text { c14 = } & 20 \\ \text { Grating length = } & 0.1 \\ \text { Grating h = } & 0.0001 \\ \text { Output coupler reflec = } & 0.98 \\ \text { Laser passes = } & 30 \\ \text { Data filename } & \text { Test } \\ & \end{array}$


// Simulation of a passive modelocking laser using a LPFG
// Department of Electronics, Carleton University 2006-2007
// Author(s): A. S. Karar
// Based on MATLAB code authored by Dr. Alan Steele and A. S. Karar

// Intrachat and Kutz, IEEE J Quant Elec 39 p1572 (2003)

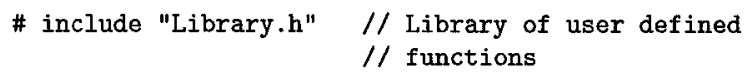

int main (void) \{

Sim sim; // Simulation object

printf("NLS Simulation --

if (!readConfiguration ( $\operatorname{sim})$ ) \{ cout $\ll "$ "In Invalid configuration file ... exit"<<endl;

printSim (sim); // display the simulation object

$/ /$ structure

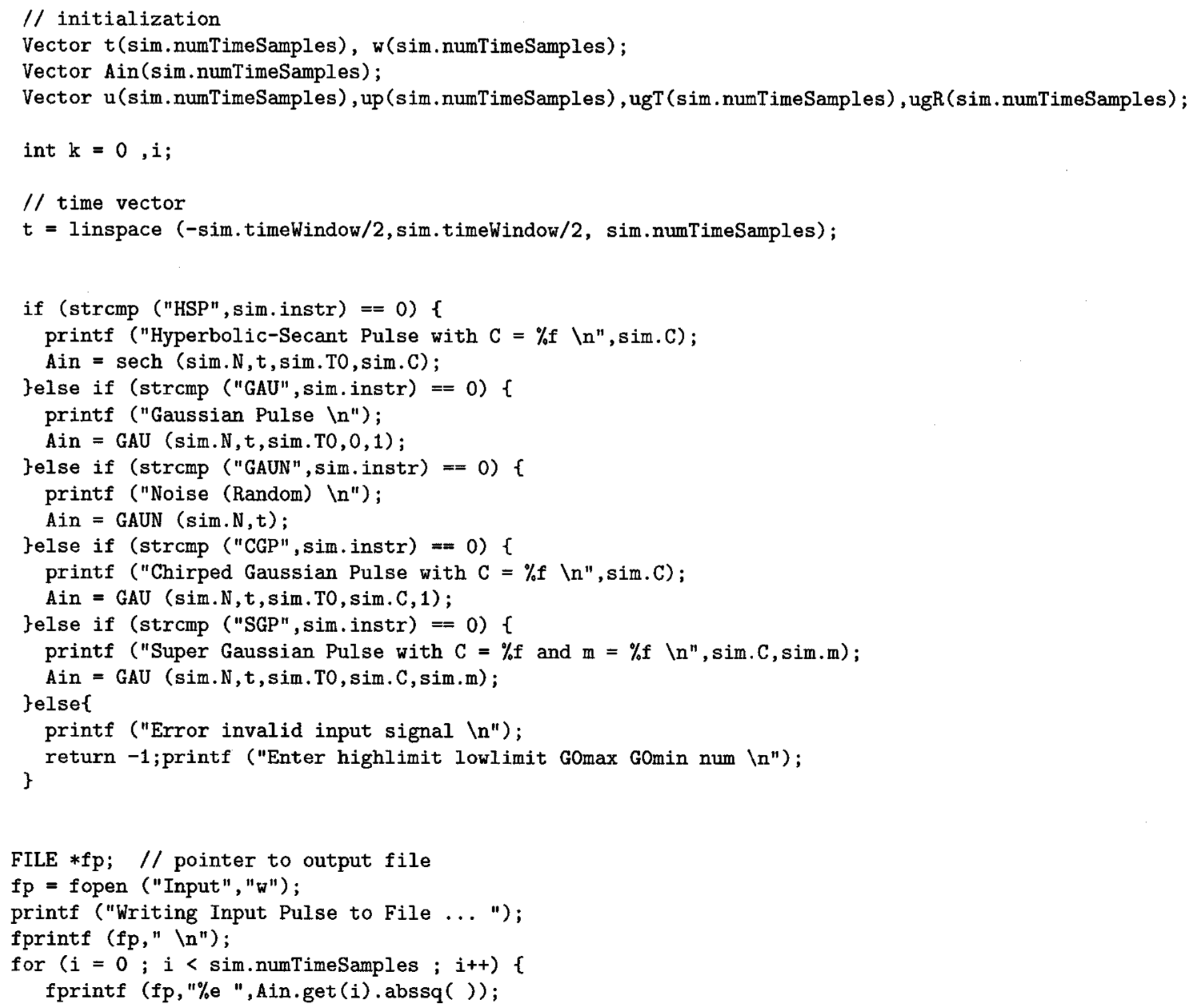


\}

fprintf (fp," $(n ")$;

fclose (fp);

printf ("Done $\backslash \mathrm{n}$ ");

$\mathrm{u}=$ Ain;

// SSFM

int $\mathrm{ms}=($ int $)(\operatorname{sim}$. fiberLength $/ \operatorname{sim} . \mathrm{h})+2 ; / / \mathrm{m}$ is the number of iterations

Complex n (0, sim. NL_gamma);

Complex d $(0,(0.5 *$ sim.beta2));

// nonlinear coefficient

Complex d3 (0,(-1.0*sim.segma)); // third order dispersion

double 1 = sim.loss_gamma;

$/ /$ third order disp
$/ /$ loss coefficient

double temp;

$/ /$ set the time vector

$t$ = linspace (-sim.timeWindow/2, sim.timeWindow/2, sim.numTimeSamples);

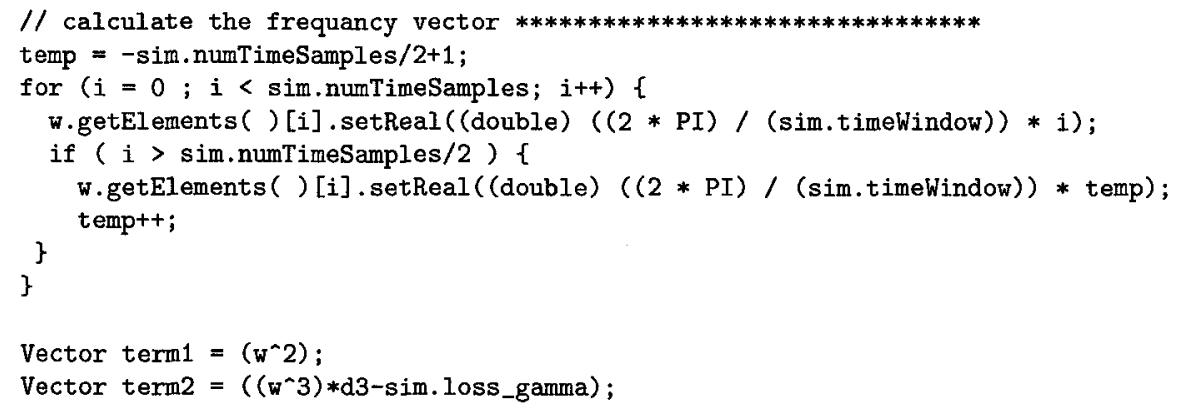

Vector Edata((highlimit-lowlimit)*num); 
Vector Gdata((highlimit-lowlimit)*num);

clock_t start $=$ clock () ;

string filename = "GO";

stringstream gvalue;

for (GO = GOmin; GO $<$ GOmax; GO = GO + GOStep $)\{$

$u=$ Ain; // new simulation

gvalue.str("");

sim.GO = G0; // set new pump power

gvalue $\ll$ G0;

$/ * * * * * * * * * *$ For OutPut $* * * * * * * * * * * * * * * * * * * * * * * * /$

$f_{p}=$ fopen $\left((f i l\right.$ ename+gvalue.str ()$\left.), c_{-} \operatorname{str}(), " w "\right)$;

fprintf (fp,"\# ");

fprintf (fp," \%d ", sim.numTimeSamples);

fprintf ( $\mathrm{f}, " \% \mathrm{~d}$ ", sim.passes);

fprintf ( $f p, " \%$ f $\backslash n "$, sim.timeWindow);

$/ * * * * * * * * * * * * * * * * * * * * * * * * * * * * * * * * * * * * * * * * * * * * * * * /$

for $(k=1 ; k<=$ sim.passes; $k++)\{$

if (strcmp ("SSFM", sim.str) $==0$ ) \{

up $=\operatorname{SSFML}(u, \operatorname{sim}, m s, n, d, d 3,1, t, w$, term 1, term 2$) ;$

\}else if (strcmp ("SSSFM", sim.str) $==0$ ) \{

up = SSSFML (u, sim,ms, $n, d, d 3,1, t, w$, term 1, term2);

\}else if (strcmp ("PCSSFM", sim.str) $==0$ ) \{

up $=\operatorname{PCSSFML}(u, \operatorname{sim}, m s, n, d, d 3,1, t, w$, term 1, term 2$) ;$

ugT = Grating (up, sim, I, sim1, sim2,mg);

ugR $=$ Grating (ugT, sim, I, sim $1, \operatorname{sim} 2, \mathrm{mg}) ;$

if (strcmp ("SSFM", sim.str) $==0$ ) \{

$\mathrm{u}=\operatorname{SSFML}(\mathrm{ugR}, \operatorname{sim}, \mathrm{ms}, \mathrm{n}, \mathrm{d}, \mathrm{d} 3,1, \mathrm{t}, \mathrm{w}, \mathrm{term} 1$, term 2 );

\}else if (strcmp ("SSSFM", sim.str) $==0$ ) \{

$u=\operatorname{SSSFML}$ (ugR, sim, ms, $n, d, d 3,1, t, w$, term 1, term 2);

\}else if (strcmp ("PCSSFM", sim.str) $==0$ ) \{

\}

$\mathrm{u}=\operatorname{PCSSFML}(\mathrm{ugR}, \operatorname{sim}, \mathrm{ms}, \mathrm{n}, \mathrm{d}, \mathrm{d} 3, \mathrm{l}, \mathrm{t}, \mathrm{w}$, term 1, term 2);

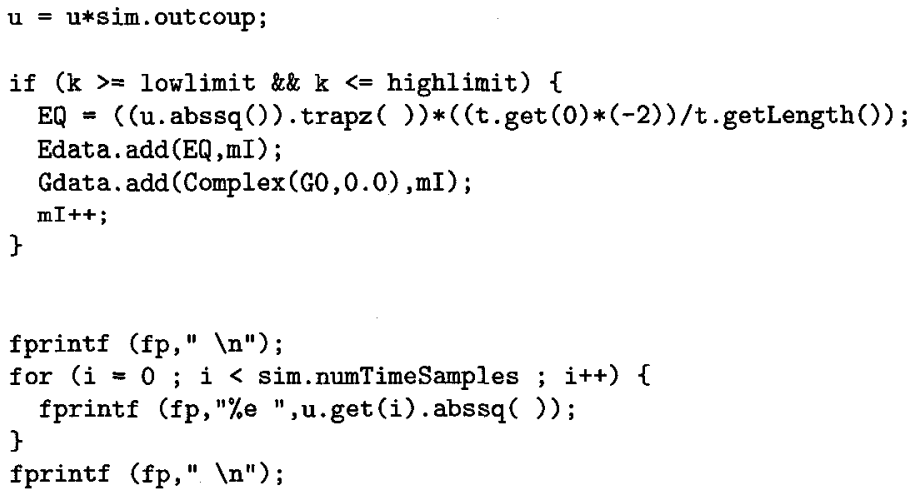


\}

fclose (fp);

\}

printf("Time elapsed: \% $\mathrm{s} \ln ",(($ double)clock () - start) / CLOCKS_PER_SEC);

$/ * * * * * * * * * *$ For OutPut $* * * * * * * * * * * * * * * * * * * * * * * * /$

fp $=$ fopen (sim.dataFile, "w");

$/ * * * * * * * * * * * * * * * * * * * * * * * * * * * * * * * * * * * * * * * * * * * * * * * * /$

printf ("Writing to file $(n ")$;

for $(\mathrm{k}=0 ; \mathrm{k}<(($ highlimit-lowlimit $) *$ num $) ; \mathrm{k}++)$ \{ \} fprintf (fp, "\%f \%f $\backslash n "$, Gdata.get (k).real,Edata.get (k).real);

fclose $(f p)$;

printf ("DONE $\backslash n ")$;

return 0 ;

\}

Reproduced with permission of the copyright owner. Further reproduction prohibited without permission. 